\title{
Wall crossing for symplectic vortices and quantum cohomology
}

\section{Journal Article}

\section{Author(s):}

Cieliebak, Kai; Salamon, Dietmar A.

Publication date:

2006

Permanent link:

https://doi.org/10.3929/ethz-b-000013339

Rights / license:

In Copyright - Non-Commercial Use Permitted

Originally published in:

Mathematische Annalen 335(1), https://doi.org/10.1007/s00208-005-0736-1 
DOI: $10.1007 /$ s00208-005-0736-1

\section{Wall crossing for symplectic vortices and quantum cohomology}

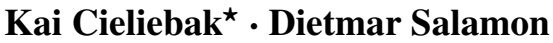

Received: 17 September 2002 / Revised 20 January 2004

Published online: 21 March 2006 - ㄷ Springer-Verlag 2006

Abstract. We derive a wall crossing formula for the symplectic vortex invariants of toric manifolds. As an application, we give a proof of Batyrev's formula for the quantum cohomology of a monotone toric manifold with minimal Chern number at least two.

\section{Introduction}

Let $T$ be a torus of dimension $k$, denote by $\mathfrak{t}$ its Lie algebra, by

$$
\Lambda:=\{\xi \in \mathfrak{t} \mid \exp (\xi)=1\}
$$

the integer lattice, and by

$$
\Lambda^{*}:=\left\{\mathrm{w} \in \mathfrak{t}^{*} \mid\langle\mathrm{w}, \xi\rangle \in \mathbb{Z} \quad \text { for } \xi \in \Lambda\right\}
$$

the dual lattice. Suppose $T$ acts diagonally on $\mathbb{C}^{n}$. The action is determined by $n$ homomorphisms $\rho_{v}: T \rightarrow S^{1}, v=1, \ldots, n$. We write each homomorphism $\rho_{v}$ in the form

$$
\rho_{\nu}(\exp (\xi))=\mathrm{e}^{-2 \pi i\left\langle\mathrm{w}_{v}, \xi\right\rangle}, \quad \mathrm{w}_{\nu} \in \Lambda^{*} .
$$

The moment map of this action, with respect to the standard symplectic form on $\mathbb{C}^{n}$, is given by

$$
\mu(x)=\pi \sum_{\nu=1}^{n}\left|x_{v}\right|^{2} \mathrm{w}_{\nu}
$$

K. Cieliebak

Ludwig-Maximilans-Universität München, Germany

(e-mail: kai@mathematik. uni-muenchen.de)

D. Salamon

ETH-Zürich, Switzerland

(e-mail: salamon@math.ethz.ch)

* Supported by National Science Foundation Grant DMS-0072267 
for $x=\left(x_{1}, \ldots, x_{n}\right) \in \mathbb{C}^{n}$. We assume throughout that $\mu$ is proper and that the vectors $\mathrm{w}_{v}$ span the space $\mathrm{t}^{*}$. In [7] we have defined, together with Gaio and Mundet, invariants

$$
\Phi_{\lambda}^{\rho, \tau}: S^{m}\left(\mathfrak{t}^{*}\right) \rightarrow \mathbb{R}, \quad m:=n-\operatorname{dim} T+\sum_{\nu=1}^{n} d_{\nu} \geq 0,
$$

by counting solutions of the genus zero symplectic vortex equations (see Section 2). Here $\lambda \in \Lambda, d_{v}:=\left\langle\mathrm{w}_{v}, \lambda\right\rangle, \tau$ is a regular value of the moment map, and $S^{m}\left(\mathfrak{t}^{*}\right)$ denotes the space of real valued polynomials of degree $m$ on $\mathfrak{t}$. Note that $S^{*}\left(\mathfrak{t}^{*}\right)$ is canonically isomorphic to the cohomology $H^{*}(B T ; \mathbb{R})$ of the classifying space $B T=E T / T$. The isomorphism takes $\mathrm{w}_{\nu} \in \mathfrak{t}^{*}$ to the first Chern class of the bundle $E T \times{ }_{\rho_{\nu}} \mathbb{C} \rightarrow B T$. The invariant $\Phi_{\lambda}^{\rho, \tau}$ takes rational values on integral cohomology classes. These correspond to polynomials that map the lattice $\Lambda$ to the integers.

An element $\tau \in \mathfrak{t}^{*}$ is a singular value of $\mu$ if and only if it can be expressed as a positive linear combination of at most $k-1$ of the vectors $\mathrm{w}_{\nu}$. The set of singular values is a disjoint union of open cones of codimensions 1 to $k$. A cone of codimension $j$ is called a wall of codimension $j$.

Let $\tau_{0} \in \mathfrak{t}^{*}$ be an element of a wall of codimension one, $\tau_{1} \in \mathfrak{t}^{*}$ be transverse to the wall at $\tau_{0}$, and $e_{1} \in \Lambda$ be the unique primitive lattice vector that is orthogonal to the wall at $\tau_{0}$ and satisfies $\left\langle\tau_{1}, e_{1}\right\rangle>0$. Denote by $T_{1} \subset T$ the subtorus generated by $e_{1}$ and by $\mathfrak{t}_{1}$ its Lie algebra. Let

$$
I:=\left\{v \mid\left\langle\mathrm{w}_{v}, e_{1}\right\rangle=0\right\} .
$$

The action $\rho$ induces an action $\rho_{0}$ of the quotient torus

$$
T_{0}:=T / T_{1}
$$

on the space

$$
\mathbb{C}^{I}:=\left\{x \in \mathbb{C}^{n} \mid x_{v}=0 \text { for } v \notin I\right\} .
$$

The moment map of this action is the restriction $\mu_{0}:=\left.\mu\right|_{\mathbb{C}^{I}}: \mathbb{C}^{I} \rightarrow \mathfrak{t}_{0}^{*}:=\mathfrak{t}_{1}^{\perp}$. The following wall crossing formula expresses the difference of the invariants on the two sides of the wall as the invariant of the reduced problem at $\tau_{0}$.

Theorem 1.1 (Genus Zero Wall Crossing). Let $\alpha \in S^{*}\left(t^{*}\right), \lambda \in \Lambda$, and $d_{v}:=$ $\left\langle\mathrm{w}_{\nu}, \lambda\right\rangle$. Then for every sufficiently small positive number $\varepsilon$ we have

$$
\Phi_{\lambda}^{\rho, \tau_{0}+\varepsilon \tau_{1}}(\alpha)-\Phi_{\lambda}^{\rho, \tau_{0}-\varepsilon \tau_{1}}(\alpha)=\Phi_{\lambda_{0}}^{\rho_{0}, \tau_{0}}\left(\alpha_{0}\right),
$$

where $\lambda_{0}$ is the projection of $\lambda$ to $\mathfrak{t}_{0}:=\mathfrak{t} / \mathfrak{t}_{1}$ and

$$
\alpha_{0}(\xi)=\frac{1}{2 \pi i} \oint \frac{\alpha\left(\xi+z e_{1}\right)}{\prod_{\nu \notin I}\left\langle\mathrm{w}_{\nu}, \xi+z e_{1}\right\rangle^{d_{v}+1}} d z .
$$

Here for each $\xi$ the integral is understood over a circle in the complex plane enclosing all the poles of the integrand. 
There is an analogous wall crossing formula for higher genus which is formulated in Theorem 3.1 below.

Theorem 1.1 gives rise to an explicit formula for the genus zero invariants. To formulate the result we introduce the following notation. For a tuple of nonnegative integers $\ell=\left(\ell_{1}, \ldots, \ell_{n}\right)$ denote

$$
\mathrm{w}^{\ell}:=\mathrm{w}_{1}^{\ell_{1}} \cdots \mathrm{w}_{n}^{\ell_{n}} \in S^{|\ell|}\left(\mathfrak{t}^{*}\right), \quad|\ell|:=\ell_{1}+\cdots+\ell_{n} .
$$

Given such a tuple $\ell$ and a lattice vector $\lambda$ we introduce the set $\mathcal{I}_{\lambda}(\ell)$ of partitions $\{1, \ldots, n\}=I_{1} \cup \cdots \cup I_{k}$ that satisfy the following two conditions.

(Dimension) For every $j \in\{1, \ldots, k\}$, the subspace

$$
E_{j}:=\operatorname{span}\left\{\mathrm{w}_{v} \mid v \in I_{1} \cup \cdots \cup I_{j}\right\} \subset \mathfrak{t}^{*}
$$

has dimension $j$ and $\mathrm{w}_{v} \notin E_{j}$ for every $v \in I_{j+1} \cup \cdots \cup I_{k}$.

(Degree) For every $j \in\{1, \ldots, k\}$,

$$
\sum_{v \in I_{j}}\left(\ell_{v}-d_{v}-1\right)=-1 . \quad d_{v}:=\left\langle\mathrm{w}_{v}, \lambda\right\rangle .
$$

Theorem 1.2 (Genus Zero Invariants). Let $\lambda \in \Lambda, d_{v}:=\left\langle\mathrm{w}_{v}, \lambda\right\rangle$, and $\ell$ be an n-tuple of nonnegative integers.

(i) If $\mathcal{I}_{\lambda}(\ell)=\emptyset$ then $\Phi_{\lambda}^{\rho, \tau}\left(\mathrm{w}^{\ell}\right)=0$ for all $\tau$.

(ii) Let $J \subset\{1, \ldots, n\}$ be a subset with $k$ elements such that $\left\{\mathrm{w}_{v} \mid v \in J\right\}$ is a basis of $\mathrm{t}^{*}$ and assume

$$
\ell_{v}= \begin{cases}d_{v} & \text { if } v \in J \\ d_{v}+1 & \text { if } v \notin J\end{cases}
$$

If $\tau$ belongs to the cone $C(J)$ spanned by $\left\{\mathrm{w}_{v} \mid v \in J\right\}$ then

$$
\Phi_{\lambda}^{\rho, \tau}\left(\mathrm{w}^{\ell}\right)=\frac{1}{\left|\operatorname{det}\left(\left\langle\mathrm{w}_{\nu}, e_{j}\right\rangle_{v \in J, j=1, \ldots, k}\right)\right|},
$$

where $e_{1}, \ldots, e_{k}$ is any basis of the lattice $\Lambda$. Otherwise $\Phi_{\lambda}^{\rho, \tau}\left(\mathrm{w}^{\ell}\right)=0$.

(iii) Let $\lambda^{\prime} \in \Lambda$ and define $d_{v}^{\prime}:=\left\langle\mathrm{w}_{v}, \lambda^{\prime}\right\rangle$. If $\ell_{v}+d_{v}^{\prime} \geq 0$ for every $v$ then

$$
\Phi_{\lambda}^{\rho, \tau}\left(\mathrm{w}^{\ell}\right)=\Phi_{\lambda+\lambda^{\prime}}^{\rho, \tau}\left(\mathrm{w}^{\ell+d^{\prime}}\right) .
$$

(iv) Assume $d_{v} \geq-1$ for every $v$. Then every element of $S^{*}\left(t^{*}\right)$ is a linear combination of monomials $\mathrm{w}^{\ell}$ that satisfy either (i) or (ii).

(v) Let $J_{\ell}:=\left\{v \mid \ell_{v} \leq d_{v}\right\}$. If $\tau \notin C\left(J_{\ell}\right)$ then $\Phi_{\lambda}^{\rho, \tau}\left(\mathrm{w}^{\ell}\right)=0$. 
Remark. Assertions (i), (ii) and (iv) can be used to compute the genus zero invariants whenever $d_{v} \geq-1$. This restriction can be removed by using (iii).

Now assume that $T$ acts freely on $\mu^{-1}(\tau)$, where

$$
\tau:=\sum_{\nu=1}^{n} \mathrm{w}_{\nu},
$$

and that the symplectic quotient

$$
\bar{M}:=\mathbb{C}^{n} / / T(\tau):=\mu^{-1}(\tau) / T
$$

has minimal Chern number $N:=\max \left\{m \in \mathbb{Z} \mid \tau / m \in \Lambda^{*}\right\} \geq 2$. These conditions guarantee that the symplectic quotient $\bar{M}$ is a monotone toric manifold. Combining Theorem 1.2 with the results of [10] one can compute the genus zero Gromov-Witten invariants of the symplectic quotient. More precisely, denote by $S^{*}\left(\mathfrak{t}^{*}\right) \rightarrow H^{*}(\bar{M} ; \mathbb{R}): \alpha \mapsto \bar{\alpha}$ the Kirwan homomorphism. Consider the dual homomorphism $H_{2}(\bar{M} ; \mathbb{Z}) \rightarrow \Lambda$ in degree two. This homomorphism is injective. We denote its image by $\Lambda(\tau)$ and the inverse map by $\Lambda(\tau) \rightarrow H_{2}(\bar{M} ; \mathbb{Z}): \lambda \mapsto \bar{\lambda}$. Given $\lambda \in \Lambda(\tau)$ we denote by $\mathrm{GW}_{\bar{\lambda}}^{\bar{M}}$ the genus zero Gromov-Witten invariant of $\bar{M}$ with fixed marked points in the homology class $\bar{\lambda}$. In [10, Theorem A] it is proved that, for every $\lambda \in \Lambda(\tau)$ and every $n$-tuple $\ell=\left(\ell_{1}, \ldots, \ell_{n}\right)$ of nonnegative integers,

$$
\Phi_{\lambda}^{\rho, \tau}\left(\mathrm{w}^{\ell}\right)=\mathrm{GW}_{\bar{\lambda}}^{\bar{M}}\left(\overline{\mathrm{w}}_{1}, \ldots, \overline{\mathrm{w}}_{1}, \ldots, \overline{\mathrm{w}}_{n}, \ldots, \overline{\mathrm{w}}_{n}\right),
$$

where each argument $\overline{\mathrm{w}}_{v}$ occurs $\ell_{v}$ times. Thus Theorem 1.2 allows us to compute the genus zero Gromov-Witten invariants of tuples of cohomology classes of degree two. This can be used to compute the quantum cohomology ring of the symplectic quotient. The statement of the theorem requires some preparation.

The chamber $C(\tau)$ is defined as the component of the set of regular values of $\mu$ that contains $\tau$. The effective cone $\Lambda_{\text {eff }}(\tau) \subset \Lambda(\tau)$ is defined as the set of lattice vectors $\lambda \in \Lambda(\tau)$ that satisfy $\left\langle\tau^{\prime}, \lambda\right\rangle \geq 0$ for every $\tau^{\prime} \in C(\tau)$.

Let $\mathcal{R}$ be any graded commutative algebra (over the reals) with unit which is equipped with a homomorphism

$$
\Lambda_{\text {eff }}(\tau) \rightarrow \mathcal{R}: \lambda \mapsto q^{\lambda}
$$

from the additive semigroup $\Lambda_{\text {eff }}(\tau)$ to the multiplicative semigroup $\mathcal{R}$ such that $\operatorname{deg}\left(q^{\lambda}\right)=2\langle\tau, \lambda\rangle$. Given such a graded algebra define the quantum cohomology ring $\mathrm{QH}^{*}(\bar{M} ; \mathcal{R})$ as the tensor product

$$
\mathrm{QH}^{*}(\bar{M} ; \mathcal{R}):=H^{*}(\bar{M} ; \mathbb{R}) \otimes \mathcal{R}
$$


(of vector spaces over the reals). Thus an element of $\mathrm{QH}^{*}(\bar{M} ; \mathcal{R})$ is a finite sum $\bar{\alpha}=\sum_{r \in \mathcal{R}} \bar{\alpha}_{r} r$ where $\bar{\alpha}_{r} \in H^{*}(\bar{M} ; \mathbb{R})$. The ring structure is defined by

$$
\bar{\alpha}^{\prime} * \bar{\alpha}^{\prime \prime}:=\sum_{i} \sum_{\lambda \in \Lambda_{\mathrm{eff}}(\tau)} \sum_{r^{\prime}, r^{\prime \prime}} \mathrm{GW}_{\bar{\lambda}}^{\bar{M}}\left(\bar{\alpha}_{r^{\prime}}^{\prime}, \bar{\alpha}_{r^{\prime \prime}}^{\prime \prime}, \bar{e}_{i}\right) \bar{e}_{i}^{*} r^{\prime} r^{\prime \prime} q^{\lambda},
$$

where the $\bar{e}_{i}$ form a basis of $H^{*}(\bar{M} ; \mathbb{R})$ and the $\bar{e}_{i}^{*}$ denote the dual basis with respect to the cup product pairing.

Theorem 1.3 (Quantum Cohomology). Let $\bar{M}=\mathbb{C}^{n} / / T(\tau)$ be a (nonempty) monotone toric manifold with minimal Chern number $N \geq 2$. Then the ring homomorphism

$$
\mathcal{R}\left[u_{1}, \ldots, u_{n}\right] \rightarrow \mathrm{QH}^{*}(\bar{M} ; \mathcal{R}): r u^{\ell} \mapsto r \overline{\mathrm{w}}^{* \ell}
$$

induces an isomorphism $\mathrm{QH}^{*}(\bar{M} ; \mathcal{R}) \cong \mathcal{R}\left[u_{1}, \ldots, u_{n}\right] / \mathcal{J}$, where the ideal $\mathcal{J} \subset$ $\mathcal{R}\left[u_{1}, \ldots, u_{n}\right]$ is generated by the relations

$$
\begin{aligned}
\sum_{v=1}^{n} \eta_{v} \mathrm{w}_{v}=0 & \Longrightarrow \sum_{\nu=1}^{n} \eta_{v} u_{v}=0, \\
\tau \notin C(\{1, \ldots, n\} \backslash\{v\}) & \Longrightarrow u_{v}=0, \\
\lambda \in \Lambda_{\text {eff }}(\tau), \quad d_{v}^{ \pm}:=\max \left\{ \pm\left\langle\mathrm{w}_{v}, \lambda\right\rangle, 0\right\} & \Longrightarrow \prod_{\nu} u_{v}^{d_{v}^{+}}=q^{\lambda} \prod_{\nu} u_{v}^{d_{v}^{-}} .
\end{aligned}
$$

The ring $\mathcal{R}\left[u_{1}, \ldots, u_{n}\right] / \mathcal{J}$ was introduced by Batyrev [4]. It also appeared in Givental's work on mirror symmetry [11] for the monotone case. Examples of Spielberg $[16,17]$ show that, in the nonmonotone case, the kernel of the homomorphism (4) is not necessarily equal to $\mathcal{J}$. For special cases the isomorphism $\mathcal{R}\left[u_{1}, \ldots, u_{n}\right] / \mathcal{J} \rightarrow \mathrm{QH}^{*}(\bar{M} ; \mathcal{R})$ was established in $[15,16]$. The reason for our hypothesis $N \geq 2$ lies in the identity (3) which, in general, does not continue to hold in the case $N=1$ (the degrees of all the classes must be less than twice the minimal Chern number).

In Section 2 we explain some background from [7] about the symplectic vortex equations. The wall crossing formula (for arbitrary genus) is restated in Section 3 and proved in Section 4. We prove Theorem 1.2 in Section 5 and Theorem 1.3 in Section 6.

\section{The symplectic vortex equations}

Fix a compact Riemann surface $\left(\Sigma, j_{\Sigma}, \mathrm{dvol}_{\Sigma}\right)$, a principal $T$-bundle $P \rightarrow \Sigma$, and an inner product on $\mathrm{t}$. The characteristic vector of $P$ will be denoted by

$$
\lambda(P):=\int_{\Sigma} F_{A} \in \Lambda
$$


Here $A \in \mathcal{A}=\mathcal{A}(P)$ is a connection on $P$ and $\lambda(P)$ is independent of the choice of the connection. For each $v \in\{1, \ldots, n\}$ the representation $\rho_{v}: T \rightarrow S^{1}$ determines a complex line bundle

$$
L_{v}:=P \times_{\rho_{v}} \mathbb{C} \rightarrow \Sigma,
$$

where the equivalence relation on $P \times \mathbb{C}$ is $[p, \zeta] \equiv\left[p g, \rho_{\nu}(g)^{-1} \zeta\right]$ for $g \in T$. This bundle has degree

$$
d_{v}:=\frac{i}{2 \pi} \dot{\rho}_{v}(\lambda(P))=\left\langle\mathrm{w}_{v}, \lambda(P)\right\rangle .
$$

A section of $L_{v}$ can be thought of as a smooth map $u_{v}: P \rightarrow \mathbb{C}$ satisfying $u_{v}(p g)=\rho_{\nu}(g)^{-1} u_{v}(p)$. The symplectic vortex equations (at a parameter $\left.\tau \in \mathfrak{t}^{*}\right)$ have the form

$$
\bar{\partial}_{A} u_{v}=0, \quad * F_{A}+\pi \sum_{\nu=1}^{n}\left|u_{\nu}\right|^{2} \mathrm{w}_{\nu}=\frac{*_{t} \lambda(P)}{\operatorname{Vol}(\Sigma)}+\tau,
$$

where $u_{v}$ is a section of $L_{v}, *: \Omega^{2}(\Sigma, \mathfrak{t}) \rightarrow \Omega^{0}\left(\Sigma, \mathfrak{t}^{*}\right)$ denotes the Hodge $*$-operator determined by the volume form on $\Sigma$ and the inner product on $\mathfrak{t}$, and $*_{\mathfrak{t}}: \mathfrak{t} \rightarrow \mathfrak{t}^{*}$ denotes the isomorphism induced by the inner product. The gauge group $\mathcal{G}:=C^{\infty}(\Sigma, T)$ acts on the space of solutions of (6) by

$$
g^{*}(A, u):=\left(A+g^{-1} d g, \rho(g)^{-1} u\right) .
$$

This action has finite isotropy if and only if $\tau$ is a regular value of $\mu$. Moreover, the moduli space

$$
\mathcal{M}(\tau):=\{(A, u) \mid u \text { and } A \text { satisfy }(6)\} / \mathcal{G}_{0}
$$

of based gauge equivalence classes of solutions of (6) is compact (see [7]). Here we fix a point $z_{0} \in \Sigma$ and denote the based gauge group by

$$
\mathcal{G}_{0}:=\left\{g \in \mathcal{G} \mid g\left(z_{0}\right)=\mathbb{1}\right\} .
$$

Think of this moduli space as a subset of the space

$$
\mathcal{B}:=\frac{\mathcal{A}(P) \times \bigoplus_{\nu=1}^{n} \Omega^{0}\left(\Sigma, L_{v}\right)}{\mathcal{G}_{0}} .
$$

The group $T$ (of constant gauge transformations) acts contravariantly on $\mathcal{B}$. Heuristically, the invariants introduced in [7] are obtained by integrating equivariant cohomology classes of $\mathcal{B}$ over $\mathcal{M}(\tau) / T$. The precise definition involves evaluating the equivariant Euler class of an associated $T$-moduli problem which we explain next. (See Appendix A for the relevant results from [8].) 
Denote

$$
\mathcal{C}:=\mathcal{A}(P) \times \bigoplus_{\nu=1}^{n} \Omega^{0}\left(\Sigma, L_{v}\right), \quad \mathcal{Z}:=\Omega^{0}\left(\Sigma, \mathfrak{t}^{*}\right) \oplus \bigoplus_{\nu=1}^{n} \Omega^{0,1}\left(\Sigma, L_{v}\right)
$$

and consider the map $\mathcal{S}: \mathcal{C} \rightarrow \mathcal{Z}$ defined by

$$
\mathcal{S}(A, u):=\left(* F_{A}+\pi \sum_{\nu=1}^{n}\left|u_{\nu}\right|^{2} \mathrm{w}_{\nu}-\frac{*_{\mathrm{t}} \lambda(P)}{\operatorname{Vol}(\Sigma)}-\tau, \bar{\partial}_{A} u_{\nu}\right) .
$$

This map is equivariant under the (contravariant) action of the gauge group $\mathcal{G}$ on both $\mathcal{C}$ and $\mathcal{Z}$ and its zero set is the space of solutions of (6). Note that the configuration space $\mathcal{C}$ is an affine space over the vector space

$$
\mathcal{X}:=\Omega^{1}(\Sigma, \mathfrak{t}) \oplus \bigoplus_{\nu=1}^{n} \Omega^{0}\left(\Sigma, L_{v}\right)
$$

Every solution $(A, u)$ of $(6)$ determines an elliptic complex

$$
0 \longrightarrow \text { Lie } \mathcal{G} \stackrel{\mathcal{L}}{\longrightarrow} \mathcal{X} \stackrel{d \mathcal{S}}{\longrightarrow} \mathcal{Z} \longrightarrow 0,
$$

where $\mathcal{L}$ is the infinitesimal action of $\mathcal{G}$ on $\mathcal{C}$ and $d \mathcal{S}$ denotes the differential of $\mathcal{S}$ at $(A, u)$. Associated to this complex is the augmented operator

$$
\mathcal{D}:=d \mathcal{S} \oplus \mathcal{K}: \mathcal{X} \rightarrow \mathcal{Z} \oplus \mathcal{V}
$$

Here $\mathcal{V}:=\Omega^{0}\left(\Sigma, t^{*}\right)$ is understood as the dual space of the Lie algebra Lie $\mathcal{G}$ and $\mathcal{K}: \mathcal{X} \rightarrow \mathcal{V}$ is defined as the $L^{2}$ adjoint of $\mathcal{L}$. Moreover, we identify the direct $\operatorname{sum} \mathcal{Z} \oplus \mathcal{V}$ with

$$
\mathcal{Y}:=\Omega^{0}\left(\Sigma, \mathfrak{t}^{*} \otimes \mathbb{C}\right) \oplus \bigoplus_{\nu=1}^{n} \Omega^{0,1}\left(\Sigma, L_{v}\right)
$$

by interpreting the first component of $\mathcal{Z}$ as the real part and the element of $\mathcal{V}$ as the imaginary part of $\Omega^{0}\left(\Sigma, t^{*} \otimes \mathbb{C}\right)$. Then the operator $\mathcal{D}$ is given by

$$
\mathcal{D}(\alpha, \hat{u}):=\left(\begin{array}{c}
* d \alpha+2 \pi \sum_{\nu=1}^{n}\left\langle u_{v}, \hat{u}_{v}\right\rangle \mathrm{w}_{v} \\
d^{*} \alpha+2 \pi \sum_{\nu=1}^{n}\left\langle i u_{v}, \hat{u}_{v}\right\rangle \mathrm{w}_{v} \\
\bar{\partial}_{A} \hat{u}_{v}+\rho_{\nu}(\alpha)^{0,1} u_{v}
\end{array}\right) .
$$

Here the first and third component correspond to the operator $d \mathcal{S}$ and the second component is the operator $\mathcal{K}$. This is a Fredholm operator of (real) Fredholm index

$$
\operatorname{index}(\mathcal{D})=(n-\operatorname{dim} T)(2-2 g)+2 \sum_{v=1}^{n} d_{v}=: 2 m,
$$


The operator $\mathcal{D}$ is complex linear, where the complex structure on $\Omega^{1}(\Sigma, \mathfrak{t})$ is given by the Hodge $*$-operator $\alpha \mapsto * \alpha=-\alpha \circ J_{\Sigma}$. Thus the determinant line of $\mathcal{D}$ inherits a natural orientation from the complex structures.

The associated $T$-moduli problem is the triple $(\mathcal{B}, \mathcal{E}, \mathcal{S})$, where the vector bundle $\mathcal{E} \rightarrow \mathcal{B}$ is given by

$$
\mathcal{E}:=\mathcal{C} \times \mathcal{G}_{0} \mathcal{Z} \rightarrow \mathcal{B}:=\mathcal{C} / \mathcal{G}_{0} .
$$

The equivariant map $\mathcal{S}: \mathcal{C} \rightarrow \mathcal{Z}$ defined by (7) descends to a section of $\mathcal{E}$ that will be denoted by the same letter. Its zero set is the above moduli space $\mathcal{M}(\tau)$. The linearized complex at a solution $(A, u)$ of (6) has the form

$$
0 \longrightarrow \mathfrak{t} \stackrel{\mathcal{L}}{\longrightarrow} \frac{\mathcal{X}}{\mathcal{L}\left(\text { Lie } \mathcal{G}_{0}\right)} \stackrel{d \mathcal{S}}{\longrightarrow} \mathcal{Z} \longrightarrow 0 .
$$

Here we identify $\mathfrak{t}$ with the space of constant maps $\Sigma \rightarrow \mathfrak{t}$. By Lemma B.4, the elliptic complexes (8) and (11) both have the same index as the operator D. Moreover, Lemma B.4 provides isomorphisms from their determinant lines to $\operatorname{det}(\mathcal{D})$. Hence the complex orientation of $\operatorname{det}(\mathcal{D})$ induces orientations of the determinant lines of (8) and (11). This is the orientation of the $T$-moduli problem $(\mathcal{B}, \mathcal{E}, \mathcal{S})$ used in [7] for the definition of the vortex invariants. (To fit this into the definitions of [8] and Appendix A, one has to replace $\mathcal{B}$ and $\mathcal{E}$ by suitable Sobolev completions.)

Evaluating the Euler class of the $T$-moduli problem $(\mathcal{B}, \mathcal{E}, \mathcal{S})$ on equivariant cohomology classes on $\mathcal{B}$ yields a homomorphism

$$
\chi^{\mathcal{B}, \mathcal{E}, \mathcal{S}}: H_{T}^{*}(\mathcal{B}) \rightarrow \mathbb{R} \text {. }
$$

Now the projection $\pi: \mathcal{B} \rightarrow \mathcal{A} / \mathcal{G}_{0}$ induces a homomorphism

$$
\pi^{*}: S^{*}\left(\mathfrak{t}^{*}\right) \otimes H^{*}\left(\mathcal{A} / \mathcal{G}_{0}\right) \cong H_{T}^{*}\left(\mathcal{A} / \mathcal{G}_{0}\right) \rightarrow H_{T}^{*}(\mathcal{B})
$$

on equivariant cohomology. Its composition with the Euler class will be denoted by

$$
\Phi_{\lambda, g}^{\rho, \tau}: S^{*}\left(\mathfrak{t}^{*}\right) \otimes H^{*}\left(\mathcal{A} / \mathcal{G}_{0}\right) \rightarrow \mathbb{R}
$$

Thus

$$
\Phi_{\lambda, g}^{\rho, \tau}(\alpha):=\chi^{\mathcal{B}, \mathcal{E}, \mathcal{S}}\left(\pi^{*} \alpha\right)=: \int_{\mathcal{M}(\tau) / T} \pi^{*} \alpha
$$

for $\alpha \in S^{*}\left(\mathfrak{t}^{*}\right) \otimes H^{*}\left(\mathcal{A} / \mathcal{G}_{0}\right) \cong H_{T}^{*}\left(\mathcal{A} / \mathcal{G}_{0}\right)$. The notation $\int_{\mathcal{M}(\tau) / T} \pi^{*} \alpha$ indicates the heuristic interpretation of the Euler class as an integral over the zero set of the section $\mathcal{S}$. This can be understood literally whenever $\mathcal{S}$ is transverse to the zero section, or equivalently, the operator $\mathcal{D}$ defined by (9) is surjective for every solution $(A, u)$ of (6). The invariant $\Phi_{\lambda, g}^{\rho, \tau}(\alpha)$ can only be nonzero when $\alpha$ has degree $2 m$, where $m$ is as in (10). 


\section{Wall crossing}

In this section we formulate the wall crossing formula for arbitrary genus at an element $\tau_{0} \in \mathfrak{t}^{*}$ of a wall of codimension one. This means that there exists an index set $I \subset\{1, \ldots, n\}$ satisfying the following conditions.

(i) The subspace $W_{I}:=\operatorname{span}\left\{\mathrm{w}_{\nu} \mid v \in I\right\} \subset \mathfrak{t}^{*}$ has dimension $k-1$ and $\mathrm{w}_{v} \notin W_{I}$ for every $v \notin I$.

(ii) $\tau_{0} \in \mu\left(\mathbb{C}^{I}\right)$, where $\mathbb{C}^{I}:=\left\{x \in \mathbb{C}^{n} \mid x_{v}=0\right.$ for $\left.v \notin I\right\}$.

(iii) If $J \subset\{1, \ldots, n\}$ is another index set satisfying (i) then $\tau_{0} \notin \mu\left(\mathbb{C}^{J}\right)$.

Note that under these conditions $\tau_{0}$ is a positive linear combination of precisely $k-1$ linearly independent vectors from the set $\left\{\mathrm{w}_{v} \mid v \in I\right\}$. Choose a vector $\tau_{1} \in \mathfrak{t}^{*}$ that is transverse to $\mu\left(\mathbb{C}^{I}\right)$ and let $e_{1} \in \Lambda$ be the unique primitive lattice vector that satisfies $\left\langle\tau_{1}, e_{1}\right\rangle>0$ and is orthogonal to the wall at $\tau_{0}$ :

$$
\left\langle\mathrm{w}_{\nu}, e_{1}\right\rangle=0 \quad \text { for } v \in I .
$$

Denote by $T_{1} \subset T$ the subtorus generated by $e_{1}$ and by $\mathfrak{t}_{1}$ its Lie algebra. Let $T_{0}:=T / T_{1}$ be the quotient torus and $\mathfrak{t}_{0}:=\mathfrak{t} / \mathfrak{t}_{1}$ be its Lie algebra. Then the action $\rho$ induces an action $\rho_{0}$ of $T_{0}$ on $\mathbb{C}^{I}$.

The wall crossing number will be expressed as an integral over the moduli space $\mathcal{M}_{0}$ of based gauge equivalence classes of solutions $\left(A,\left\{u_{v}\right\}_{v \in I}\right)$ of the equations

$$
\bar{\partial}_{A} u_{v}=0 \quad(v \in I), \quad * F_{A}+\pi \sum_{\nu \in I}\left|u_{\nu}\right|^{2} \mathrm{w}_{\nu}=\frac{{ }{ }_{t} \lambda(P)}{\operatorname{Vol}(\Sigma)}+\tau_{0} .
$$

We shall view this as a $T_{0}$-moduli problem. Indeed, the subgroup $T_{1} \subset T$ acts trivially on $\mathcal{M}_{0}$. However, since $\tau_{0}$ is a regular value of $\left.\mu\right|_{\mathbb{C}^{I}}$, the quotient group $T_{0}=T / T_{1}$ acts on $\mathcal{M}_{0}$ with finite isotropy.

It is interesting to compare $\mathcal{M}_{0}$ with the moduli space $\mathcal{M}\left(\mathbb{C}^{I}, P_{0}, \tau_{0}\right)$ of based gauge equivalence classes of solutions of (6) with $\mathbb{C}^{n}, T$, and $P$ replaced $\mathbb{C}^{I}$, $T_{0}:=T / T_{1}$, and $P_{0}:=P / T_{1}$. There is a natural projection

$$
\mathcal{M}_{0} \longrightarrow \mathcal{M}\left(\mathbb{C}^{I}, P_{0}, \tau_{0}\right):\left[A,\left\{u_{v}\right\}_{v \in I}\right] \mapsto\left[A_{0},\left\{u_{v}\right\}_{v \in I}\right],
$$

where $A_{0}:=\Pi_{0} A \in \Omega^{1}\left(P, \mathfrak{t}_{0}\right)$ can be thought of as a connection on $P_{0}$. Here $\Pi_{0}: \mathfrak{t} \rightarrow \mathfrak{t}_{0}$ denotes the canonical projection. If $\left(A,\left\{u_{v}\right\}_{v \in I}\right)$ satisfies (13) then the tuple $\left(A_{0},\left\{u_{v}\right\}_{v \in I}\right)$ satisfies the equations

$$
\bar{\partial}_{A_{0}} u_{v}=0 \quad(v \in I), \quad *_{0} F_{A_{0}}+\pi \sum_{\nu \in I}\left|u_{v}\right|^{2} \mathrm{w}_{v}=\frac{*_{\mathrm{t}_{0}} \lambda\left(P_{0}\right)}{\operatorname{Vol}(\Sigma)}+\tau_{0} .
$$

and hence belongs to the moduli space $\mathcal{M}\left(\mathbb{C}^{I}, P_{0}, \tau_{0}\right)$. Here $*_{\mathfrak{t}_{0}}: \mathfrak{t} / \mathfrak{t}_{1} \rightarrow \mathfrak{t}_{1}^{\perp}$ is given by $[\xi] \mapsto \xi-\left|e_{1}\right|^{-2}\left\langle\xi, e_{1}\right\rangle e_{1}$ and $*_{0}: \Omega^{2}\left(\Sigma, \mathfrak{t} / \mathfrak{t}_{1}\right) \rightarrow \Omega^{0}\left(\Sigma, \mathfrak{t}_{1}^{\perp}\right)$ is induced 
by the Hodge $*$-operator on $\Sigma$ and $*_{t_{0}}$. The map (14) defines a fibration whose fiber can be described as follows.

Choose a complement of $\mathfrak{t}_{1}$ in $\mathfrak{t}$ and denote the resulting projection by $\Pi_{1}$ : $\mathfrak{t} \rightarrow \mathfrak{t}_{1}$. Define Pic $_{1}$ as the space of based gauge equivalence classes of real valued $T$-invariant 1 -forms $A_{1} \in \Omega^{1}(P)$ that satisfy $A_{1}\left(p e_{1}\right)=1$ for every $p \in P$ and

$$
* F_{A_{1}}=\frac{\Pi_{1} \lambda(P)}{\operatorname{Vol}(\Sigma)} .
$$

Here the based gauge group is $\mathcal{G}_{1}:=\left\{g_{1}: \Sigma \rightarrow T_{1} \mid g_{1}\left(z_{0}\right)=\mathbb{1}\right\}$ and it acts by $\left(g_{1}{ }^{*} A_{1}\right) e_{1}:=A_{1} e_{1}+g_{1}{ }^{-1} d g_{1}$. Note that $\mathrm{Pic}_{1}$ is a $2 g$-torus. It is the fibre in (14) because the subgroup $\mathcal{G}_{1} \subset \mathcal{G}_{0}$ acts trivially on $A_{0}=\Pi_{0} A$ and $u_{v}$ for every $v \in I$. We emphasize that the fibration (14) need not be a product. The reason is that the map $A \mapsto A_{1}:=\Pi_{1} A$ will not, in general, be gauge invariant. A situation analogous to the one described by (13) and (14) was considered in [14].

We are now in a position to state the wall crossing formula for arbitrary genus. Think of $\mathcal{M}_{0}$ as a subset of the space $\mathcal{B}_{0}:=\mathcal{A}(P) \times C_{T}^{\infty}\left(P, \mathbb{C}^{I}\right) / \mathcal{G}_{0}$ and denote by

$$
\pi_{0}^{*}: S^{*}\left(\mathfrak{t}_{0}^{*}\right) \otimes H^{*}\left(\mathcal{A} / \mathcal{G}_{0}\right) \rightarrow H_{T_{0}}^{*}\left(\mathcal{B}_{0}\right)
$$

the homomorphism on equivariant cohomology induced by the $T_{0}$-invariant projection $\pi_{0}: \mathcal{B}_{0} \rightarrow \mathcal{A} / \mathcal{G}_{0}$. The wall crossing formula involves integration of $T_{0}$-equivariant cohomology classes on $\mathcal{B}_{0}$ over $\mathcal{M}_{0} / T_{0}$. This is to be understood as the evaluation of the Euler class of the associated $T_{0}$-moduli problem.

Theorem 3.1 (Wall Crossing). Let $\alpha \in S^{*}\left(\mathfrak{t}^{*}\right) \otimes H^{*}\left(\mathcal{A} / \mathcal{G}_{0}\right), \lambda \in \Lambda$, and $d_{v}:=$ $\left\langle\mathrm{w}_{v}, \lambda\right\rangle$.

Then, for every sufficiently small positive number $\varepsilon$, we have

$$
\Phi_{\lambda, g}^{\rho, \tau_{0}+\varepsilon \tau_{1}}(\alpha)-\Phi_{\lambda, g}^{\rho, \tau_{0}-\varepsilon \tau_{1}}(\alpha)=\int_{\mathcal{M}_{0} / T_{0}} \pi_{0}^{*} \alpha_{0},
$$

where $\alpha_{0} \in S^{*}\left(\mathfrak{t}_{0}^{*}\right) \otimes H^{*}\left(\mathcal{A} / \mathcal{G}_{0}\right)$ is the polynomial map defined by

$$
\alpha_{0}(\xi):=\frac{1}{2 \pi i} \oint \frac{\alpha\left(\xi+z e_{1}\right)}{\prod_{\nu \notin I}\left\langle\mathrm{w}_{\nu}, \xi+z e_{1}\right\rangle^{d_{\nu}+1-g}} \exp \left(\sum_{\nu \notin I} \frac{\Omega_{\nu}}{\left\langle\mathrm{w}_{\nu}, \xi+z e_{1}\right\rangle}\right) d z .
$$

Here $\Omega_{v}$ is the closed 2-form on $\mathcal{A} / \mathcal{G}_{0}$ defined by

$$
\Omega_{v}:=\sum_{j, j^{\prime}=1}^{k} \Omega_{j j^{\prime}} \mathrm{W}_{v j} \mathrm{w}_{v j^{\prime}}
$$


Here $e_{1}, \ldots, e_{k}$ is a basis of $\mathfrak{t}$ and $\alpha_{1}, \ldots, \alpha_{2 g}$ is a symplectic basis of $H^{1}(\Sigma ; \mathbb{Z})$. These bases induce a basis $\tau_{i j}$ of $H^{1}\left(\mathcal{A} / \mathcal{G}_{0} ; \mathbb{Z}\right)$ and $\Omega_{j j^{\prime}}$ and $\mathrm{w}_{v j}$ are defined by

$$
\Omega_{j j^{\prime}}:=\sum_{i=1}^{g} \tau_{i j} \wedge \tau_{i+g, j^{\prime}}, \quad \mathrm{w}_{\nu j}:=\left\langle\mathrm{w}_{\nu}, e_{j}\right\rangle .
$$

Note that $\Omega_{j j^{\prime}}$ is independent of the choice of the $\alpha_{i}$ and $\Omega_{\nu}$ is independent of the choice of both bases. For each $\xi$, the integral in the definition of $\alpha_{0}(\xi)$ is over a circle in the complex plane enclosing all the poles of the integrand.

Remark 3.2 (Residues). Consider a rational function $f: \mathbb{C} \rightarrow \mathbb{C}$ with poles $p_{1}, \ldots, p_{n}$. It induces a meromorphic 1-form $f d z$. Let $\oint f d z$ be the integral of $f d z$ over a closed curve in $\mathbb{C}$ around all the poles of $f$. By the residue theorem,

$$
\frac{1}{2 \pi i} \oint f d z=-\operatorname{Res}_{\infty}(f d z)=\sum_{j=1}^{n} \operatorname{Res}_{p_{j}}(f d z) .
$$

Note that the 1-form $f d z$ and hence the residue at infinity do not change if the complex coordinate is shifted by $z \mapsto z+c$. If we expand $f$ as a Laurent series

$$
f(z)=\sum_{k=-\infty}^{k_{0}} a_{k} z^{k}
$$

in $z^{-1}$ that converges near infinity, then the residue at infinity is minus the coefficient of $z^{-1}$, i.e. $\operatorname{Res}_{\infty}(f)=-a_{-1}$.

Example 3.3. Consider the action of the 1-torus $T=\mathbb{R} / \mathbb{Z}$ on $\mathbb{C}^{n}$ with positive integer weights $\mathrm{w}_{v}=\ell_{v} \in \Lambda^{*} \cong \mathbb{Z}$. Thus the symplectic quotient in the nontrivial chamber is a weighted projective space. Let $c \in \Lambda^{*}$ be the standard generator $c(\xi)=\xi$ and pick a homology class $\lambda=d \in \Lambda \cong \mathbb{Z}$. Assume

$$
m:=\sum_{\nu=1}^{n}\left(d \ell_{\nu}+1-g\right)+g-1 \geq 0 .
$$

We compute the invariant $\Phi_{d, g}^{\rho, \tau}\left(c^{m}\right)$ in the nonempty chamber by wall crossing from the empty chamber. Here $I=\emptyset, T_{1}=T=\mathbb{R} / \mathbb{Z}, e_{1}=1$, and $T_{0}=\{11\}$. Then $\mathcal{M}_{0}=$ Pic is a $2 g$-torus and $\Omega_{v}=\ell_{v}{ }^{2} \Omega$, where $\Omega$ is the standard symplectic form on Pic. It satisfies

$$
\frac{1}{g !} \int_{\mathrm{Pic}} \Omega^{g}=1
$$


The integrand in Theorem 3.1 is given by

$$
\begin{aligned}
\alpha_{0} & =\frac{1}{2 \pi i} \oint \frac{z^{m}}{\prod_{\nu}\left(\ell_{v} z\right)^{d \ell_{v}+1-g}} \exp \left(\frac{\left(\ell_{1}+\cdots+\ell_{n}\right) \Omega}{z}\right) \\
& =\frac{\left(\ell_{1}+\cdots+\ell_{n}\right)^{g}}{\prod_{\nu=1}^{n} \ell_{\nu}^{d \ell_{\nu}+1-g}} \frac{\Omega^{g}}{g !} .
\end{aligned}
$$

Integrating this class over Pic yields the formula from [7]:

$$
\Phi_{\lambda, g}^{\rho, \tau}\left(c^{m}\right)=\frac{\left(\ell_{1}+\cdots+\ell_{n}\right)^{g}}{\prod_{\nu=1}^{n} \ell_{v}^{d \ell_{v}+1-g}} .
$$

\section{Proof of the wall crossing formula}

\subsection{A cobordism argument}

The idea of the proof of Theorem 3.1 is to study the cobordism $\mathcal{W}$ from $\mathcal{M}\left(\tau_{0}-\varepsilon \tau_{1}\right)$ to $\mathcal{M}\left(\tau_{0}+\varepsilon \tau_{1}\right)$ constructed from the solutions of (6) with $\tau$ varying from $\tau_{0}-\varepsilon \tau_{1}$ to $\tau_{0}+\varepsilon \tau_{1}$. If this cobordism were a manifold the wall crossing number would be zero. However, in general, the cobordism $\mathcal{W}$ will have a singular set $\mathcal{M}_{0}$ associated to the value $\tau=\tau_{0}$. We shall prove the wall crossing formula by cutting out a neighbourhood of the singular set and evaluating the Euler class asssociated to the resulting third boundary component $\mathcal{M}_{\delta}$. It turns out that for the computation it is easier to deform $\mathcal{M}_{\delta}$ first into another $T$-moduli problem which we explain next.

We introduce the gauge invariant differential equations

$$
\begin{aligned}
\bar{\partial}_{A} u_{v}=0 \quad(v=1, \ldots, n), \\
* F_{A}+\pi \sum_{\nu \in I}\left|u_{\nu}\right|^{2} \mathrm{w}_{v}=\frac{*_{t} \lambda(P)}{\operatorname{Vol}(\Sigma)}+\tau_{0}, \quad \sum_{\nu \notin I}\left\|u_{v}\right\|^{2}=1,
\end{aligned}
$$

where $\left\|u_{v}\right\|$ denotes the $L^{2}$-norm. Denote the moduli space of based gauge equivalence classes of solutions of (17) by

$$
\mathcal{P}_{0}:=\{(A, u) \mid A \text { and } u \text { satisfy }(17)\} / \mathcal{G}_{0} .
$$

Note that there is a $T$-equivariant projection $\mathcal{P}_{0} \rightarrow \mathcal{M}_{0}$ whose preimage at each point $\left(A,\left\{u_{v}\right\}_{v \in I}\right) \in \mathcal{M}_{0}$ is the unit sphere in the kernel of the Cauchy-Riemann operator in the variables $u_{v}$ for $v \notin I$. We emphasize that the dimensions of these preimages can vary even if $\mathcal{P}_{0}$ is a manifold.

The $T$-moduli problem associated to $(17)$ is the triple $\left(\mathcal{B}, \mathcal{E}_{0}, \mathcal{S}_{0}\right)$ defined as follows. The base $\mathcal{B}$ is defined by $\mathcal{B}:=\mathcal{C} / \mathcal{G}_{0}$ as in Section 2 . Denote by $\mathcal{V}_{0} \subset \mathcal{V}$ the codimension- 1 subspace

$$
\mathcal{V}_{0}:=\left\{\xi \in \Omega^{0}\left(\Sigma, \mathfrak{t}^{*}\right) \mid \int_{\Sigma}\left\langle\xi, e_{1}\right\rangle \operatorname{dvol}_{\Sigma}=0\right\},
$$


and by $\mathcal{Z}_{0} \subset \mathcal{Z}$ the codimension- 1 subspace

$$
\mathcal{Z}_{0}:=\mathcal{V}_{0} \oplus \bigoplus_{\nu=1}^{n} \Omega^{0,1}\left(\Sigma, L_{\nu}\right)
$$

Then the vector bundle $\mathcal{E}_{0} \rightarrow \mathcal{B}$ is given by

$$
\mathcal{E}_{0}:=\mathcal{C} \times \mathcal{G}_{0}\left(\mathcal{Z}_{0} \oplus \mathbb{R}\right) .
$$

As above we do not distinguish in notation between a $T$-equivariant section $\mathcal{B} \rightarrow \mathcal{E}_{0}$ and its lift to a $\mathcal{G}$-equivariant function $\mathcal{C} \rightarrow \mathcal{Z}_{0} \oplus \mathbb{R}$. The section $\mathcal{S}_{0}: \mathcal{C} \rightarrow \mathcal{Z}_{0} \oplus \mathbb{R}$ is defined by

$$
\mathcal{S}_{0}(A, u):=\left(* F_{A}+\pi \sum_{\nu \in I}\left|u_{\nu}\right|^{2} \mathrm{w}_{\nu}-\frac{*_{\mathfrak{t}} \lambda(P)}{\operatorname{Vol}(\Sigma)}-\tau_{0}, \bar{\partial}_{A} u_{v}, \sum_{\nu \notin I}\left\|u_{\nu}\right\|^{2}-1\right) .
$$

In the following proposition the integral is understood as $\chi^{\mathcal{B}, \mathcal{E}_{0}, \mathcal{S}_{0}}\left(\pi^{*} \alpha\right)$, where the orientation of the $T$-moduli problem $\left(\mathcal{B}, \mathcal{E}_{0}, \mathcal{S}_{0}\right)$ is as in Remark 4.2 below.

Proposition 4.1. The wall crossing number can be expressed in the form

$$
\Phi_{\lambda, g}^{\rho, \tau_{0}+\varepsilon \tau_{1}}(\alpha)-\Phi_{\lambda, g}^{\rho, \tau_{0}-\varepsilon \tau_{1}}(\alpha)=\int_{\mathcal{P}_{0} / T} \pi^{*} \alpha .
$$

Remark 4.2 (Orientation). The moduli problem $\left(\mathcal{B}, \mathcal{E}_{0}, \mathcal{S}_{0}\right)$ is oriented as follows. The elliptic complex associated to a solution $(A, u)$ of (17) has the form

$$
0 \longrightarrow \mathfrak{t} \stackrel{\mathcal{L}}{\longrightarrow} \frac{\mathcal{X}}{\mathcal{L}\left(\text { Lie } \mathcal{G}_{0}\right)} \stackrel{d \mathcal{S}_{0}}{\longrightarrow} \mathcal{Z}_{0} \oplus \mathbb{R} \longrightarrow 0 .
$$

Since $\mathcal{L}$ is injective, the derminant line of this complex is equal to the determinant line of the Fredholm operator

$$
d \mathcal{S}_{0}: \frac{\mathcal{X}}{\mathcal{L}(\operatorname{Lie} \mathcal{G})} \rightarrow \mathcal{Z}_{0} \oplus \mathbb{R}
$$

Define the operator $\mathcal{K}_{0}: \mathcal{X} \rightarrow \mathcal{V}_{0} \oplus \mathbb{R}$ by

$$
\mathcal{K}_{0}(\alpha, \hat{u}):=\left(d^{*} \alpha+2 \pi \sum_{\nu \in I}\left\langle i u_{v}, \hat{u}_{v}\right\rangle \mathrm{w}_{\nu}, \operatorname{Im} \Psi(\hat{u})\right),
$$

where the complex linear map $\Psi: \bigoplus_{v=1}^{n} \Omega^{0}\left(\Sigma, L_{v}\right) \rightarrow \mathbb{C}$ is defined by

$$
\Psi(\hat{u}):=\frac{2 \pi}{\left\langle\tau_{1}, e_{1}\right\rangle \operatorname{Vol}(\Sigma)} \sum_{\nu \notin I}\left\langle\mathrm{w}_{\nu}, e_{1}\right\rangle \int_{\Sigma}\left(\left\langle u_{v}, \hat{u}_{v}\right\rangle+i\left\langle i u_{v}, \hat{u}_{v}\right\rangle\right) \operatorname{dvol}_{\Sigma} .
$$

The imaginary part of $\Psi$ corresponds to the local slice for the $T_{1}$-action. It follows that $\mathcal{K}_{0}$ is surjective and its kernel is a complement of the image of the 
infinitesimal action $\mathcal{L}: \operatorname{Lie}(\mathcal{G}) \rightarrow \mathcal{X}$. Hence Lemma B.4 provides an isomorphism from the determinant line of the augmented operator $d \mathcal{S}_{0} \oplus \mathcal{K}_{0}$ to the determinant line of the complex (21). Thus it remains to orient the operators $d \mathcal{S}_{0} \oplus \mathcal{K}_{0}: \mathcal{X} \rightarrow \mathcal{Z}_{0} \oplus \mathbb{R} \oplus \mathcal{V}_{0} \oplus \mathbb{R}$ in a consistent way.

For this it is convenient to introduce the operator $\mathcal{D}_{0}: \mathcal{X} \rightarrow \mathcal{Y}$ by

$$
\mathcal{D}_{0}(\alpha, \hat{u}):=\left(\begin{array}{c}
* d \alpha+2 \pi \sum_{v \in I}\left\langle u_{v}, \hat{u}_{v}\right\rangle \mathrm{w}_{v} \\
d^{*} \alpha+2 \pi \sum_{v \in I}\left\langle i u_{\nu}, \hat{u}_{v}\right\rangle \mathrm{w}_{v} \\
\bar{\partial}_{A} \hat{u}_{v}+\rho_{v}(\alpha)^{0,1} u_{v}
\end{array}\right) .
$$

As in Section 2, the first two components denote the real and imaginary parts in $\Omega^{0}\left(\Sigma, \mathfrak{t}^{*} \otimes \mathbb{C}\right)$ and $\mathcal{D}_{0}$ is complex linear. Note that the image of $\mathcal{D}_{0}$ is always contained in the complex codimension-1 subspace

$$
\mathcal{Y}_{0}:=\left\{\xi \in \Omega^{0}\left(\Sigma, \mathrm{t}^{*} \otimes \mathbb{C}\right) \mid \int_{\Sigma}\left\langle\xi, e_{1}\right\rangle \mathrm{dvol}_{\Sigma}=0\right\} \oplus \bigoplus_{\nu=1}^{n} \Omega^{0,1}\left(\Sigma, L_{\nu}\right) .
$$

Define the linear map $\Phi: \mathcal{X} \rightarrow \mathbb{R}^{2}$ by

$$
\Phi(\alpha, \hat{u}):=\left(\sum_{\nu \notin I} \int_{\Sigma}\left\langle u_{\nu}, \hat{u}_{\nu}\right\rangle \mathrm{dvol}_{\Sigma}, \operatorname{Im} \Psi(\hat{u})\right) .
$$

Then the operator $\mathcal{D}_{0} \oplus \Phi: \mathcal{X} \rightarrow \mathcal{Y}_{0} \oplus \mathbb{R}^{2}$ is equal to $d \mathcal{S}_{0} \oplus \mathcal{K}_{0}$ : $\mathcal{X} \rightarrow \mathcal{Z}_{0} \oplus \mathbb{R} \oplus \mathcal{V}_{0} \oplus \mathbb{R}$ under the obvious identifications. Since $\mathcal{D}_{0}$ is complex linear the resulting orientation of $\operatorname{det}\left(\mathcal{D}_{0}\right)$ induces, by Lemma B.1, an orientation of $\operatorname{det}\left(\mathcal{D}_{0} \oplus \Phi\right)$ and hence of the complex (21). Note that the orientation depends on $e_{1}$ : if $e_{1}$ changes sign, then so does the imaginary part of $\Psi$ and hence, by Lemma B.3, the orientation of our $T$-moduli problem.

Remark 4.3. If the operator $\mathcal{D}_{0} \oplus \Phi: \mathcal{X} \rightarrow \mathcal{Y}_{0} \oplus \mathbb{R}^{2}$ in Remark 4.2 is onto then $\mathcal{P}_{0}$ is a smooth $T$-invariant submanifold of $\mathcal{B}$ near $(A, u)$ and the tangent space of the quotient $\mathcal{P}_{0} / T$ at $(A, u)$ is

$$
T_{[A, u]} \mathcal{P}_{0} / T=\left\{(\alpha, \hat{u}) \mid \mathcal{D}_{0}(\alpha, \hat{u})=0, \Phi(\alpha, \hat{u})=0\right\} .
$$

In this case a basis $v_{1}, \ldots, v_{2 m}$ of the tangent space is positively oriented if the vectors $v_{1}, \ldots, v_{2 m}, w_{0}:=\left(0,\left\{u_{v}\right\}_{\nu \notin I}\right), w_{1}:=\left(0,\left\{2 \pi i\left\langle\mathrm{w}_{\nu}, e_{1}\right\rangle u_{v}\right\}_{v \notin I}\right)$ form a positive basis of the complex vector space ker $\mathcal{D}_{0}$.

Proof of Proposition 4.1. Denote $\tau_{t}:=\tau_{0}+t \tau_{1}$ and consider the moduli space

$$
\mathcal{W}:=\left\{(t, A, u) \mid-\varepsilon \leq t \leq \varepsilon,(6) \text { holds with } \tau=\tau_{t}\right\} / \mathcal{G}_{0} .
$$

This space has boundary

$$
\partial \mathcal{W}=\left(\{-\varepsilon\} \times \mathcal{M}\left(\tau_{-\varepsilon}\right)\right) \cup\left(\{\varepsilon\} \times \mathcal{M}\left(\tau_{\varepsilon}\right)\right) .
$$


Note that $\mathcal{W}$ is the zero set of the $T$-moduli problem with boundary $([-\varepsilon, \varepsilon] \times$ $\mathcal{B},[-\varepsilon, \varepsilon] \times \mathcal{E},\left\{\mathcal{S}_{t}\right\}_{-\varepsilon \leq t \leq \varepsilon}$ ), where $\mathcal{S}_{t}$ is defined by (7) with $\tau=\tau_{t}$. This moduli problem will in general not be regular.

The isotropy subgroup of an element $[t, A, u] \in \mathcal{W}$ is not finite if and only if $t=0$ and $u_{v}=0$ for $v \notin I$. This singular set is the moduli space $\mathcal{M}_{0}$ introduced in Section 3. To obtain a regular cobordism we cut out a neighbourhood of the singular set. Thus we consider the configuration space

$$
\mathcal{Q}:=\left\{(t, A, u) \in[-\varepsilon, \varepsilon] \times \mathcal{C} \mid \sum_{\nu \notin I}\left\|u_{\nu}\right\|^{2} \geq \delta\right\},
$$

where $\|\cdot\|$ denotes the $L^{2}$-norm. Define the $T$-moduli problem $(\mathcal{R}, \mathcal{F}, \mathcal{T})$ by

$$
\mathcal{R}:=\mathcal{Q} / \mathcal{G}_{0}, \quad \mathcal{F}:=\mathcal{Q} \times \mathcal{G}_{0} \mathcal{Z}, \quad \mathcal{T}(t, A, u):=\mathcal{S}_{t}(A, u) .
$$

The boundary of this moduli problem has three parts: $\left(\mathcal{B}, \mathcal{E}, \mathcal{S}_{-\varepsilon}\right)$ for $t=-\varepsilon$, $\left(\mathcal{B}, \mathcal{E}, \mathcal{S}_{\varepsilon}\right)$ for $t=\varepsilon$, and $\left(\mathcal{B}^{\delta}, \mathcal{E}^{\delta}, \mathcal{S}^{\delta}\right)$ associated to the condition $\sum_{\nu \notin I}\left\|u_{\nu}\right\|^{2}=\delta$. These three boundary strata intersect and so $(\mathcal{R}, \mathcal{F}, \mathcal{T})$ is a $T$-moduli problem with corners. However, we shall see that, if $\delta>0$ is sufficiently small, the zero set $\mathcal{W}^{\delta}$ of $\mathcal{T}$ does not intersect the corners and hence we obtain a $T$-moduli problem with boundary by restricting to a sufficiently small neighbourhood of $\mathcal{W}^{\delta}$. Namely, the intersection of $\mathcal{W}^{\delta}$ with the boundary of $\mathcal{R}$ is the set

$$
\partial \mathcal{W}^{\delta}=\left(\{-\varepsilon\} \times \mathcal{M}\left(\tau_{-\varepsilon}\right)\right) \cup\left(\{\varepsilon\} \times \mathcal{M}\left(\tau_{\varepsilon}\right)\right) \cup \mathcal{M}^{\delta},
$$

where $\mathcal{M}^{\delta}$ is the moduli space of based gauge equivalence classes of solutions of

$$
\begin{aligned}
\bar{\partial}_{A} u_{\nu}=0 \quad(v=1, \ldots, n), \\
* F_{A}+\pi \sum_{\nu=1}^{n}\left|u_{\nu}\right|^{2} \mathrm{w}_{\nu}=\frac{*_{t} \lambda(P)}{\operatorname{Vol}(\Sigma)}+\tau_{t}, \quad \sum_{\nu \notin I}\left\|u_{\nu}\right\|^{2}=\delta .
\end{aligned}
$$

Every solution of these equations satisfies

$$
t=\frac{\pi}{\left\langle\tau_{1}, e_{1}\right\rangle \operatorname{Vol}(\Sigma)} \sum_{\nu \notin I}\left\|u_{\nu}\right\|^{2}\left\langle\mathrm{w}_{\nu}, e_{1}\right\rangle .
$$

To see this take the inner product of the second equation in (25) with $e_{1}$ and integrate over $\Sigma$. This shows that the parameter $t$ is determined by $u$ and can therefore be removed in the definition of $\mathcal{M}^{\delta}$. Moreover, the formula (26) shows that $|t|<\varepsilon$ whenever $\delta$ is sufficiently small.

We define the moduli problem $\left(\mathcal{B}^{\delta}, \mathcal{E}^{\delta}, \mathcal{S}^{\delta}\right)$ by

$$
\mathcal{B}^{\delta}:=\mathcal{C}^{\delta} / \mathcal{G}_{0}, \quad \mathcal{E}^{\delta}:=\mathcal{C}^{\delta} \times \mathcal{G}_{0} \mathcal{Z}_{0}, \quad \mathcal{C}^{\delta}:=\left\{(A, u) \in \mathcal{C} \mid \sum_{\nu \notin I}\left\|u_{\nu}\right\|^{2}=\delta\right\},
$$


where $\mathcal{Z}_{0}$ is defined by (19). The section $\mathcal{S}^{\delta}: \mathcal{C}^{\delta} \rightarrow \mathcal{Z}_{0}$ is defined by $\mathcal{S}^{\delta}(A, u)$ $:=\mathcal{T}(t, A, u)$, where $t$ is given by (26). There is an obvious morphism of this moduli problem to the restriction of $(\mathcal{R}, \mathcal{F}, \mathcal{T})$ to the boundary component $\sum_{v \notin I}\left\|u_{v}\right\|^{2}$ $=\delta$, given by $(u, A) \mapsto(t, u, A)$ with $t$ given by (26).

Throughout we shall work with the opposite boundary orientation of the moduli problem $\left(\mathcal{B}^{\delta}, \mathcal{E}^{\delta}, \mathcal{S}^{\delta}\right)$. We begin by explaining how it is defined. The elliptic complex at a point $(A, u) \in \mathcal{M}^{\delta}$ has the form

$$
0 \longrightarrow \mathfrak{t} \stackrel{\mathcal{L}}{\longrightarrow} \frac{\mathcal{X}^{\delta}}{\mathcal{L}\left(\text { Lie } \mathcal{G}_{0}\right)} \stackrel{d \mathcal{S}^{\delta}}{\longrightarrow} \mathcal{Z}_{0} \longrightarrow 0,
$$

where $\mathcal{X}^{\delta}:=T_{(A, u)} \mathcal{C}^{\delta} \subset \mathcal{X}$ is the real codimension-1 subspace

$$
\mathcal{X}^{\delta}:=\left\{(\alpha, \hat{u}) \in \mathcal{X} \mid \sum_{\nu \notin I} \int_{\Sigma}\left\langle u_{\nu}, \hat{u}_{v}\right\rangle \mathrm{dvol}_{\Sigma}=0\right\} .
$$

We show how to identify the determinant of the complex (27) with the determinant of the operator

$$
\mathcal{D}_{1} \oplus \Phi: \mathcal{X} \longrightarrow \mathcal{Y}_{0} \oplus \mathbb{R}^{2},
$$

where $\Phi: \mathcal{X} \rightarrow \mathbb{R}^{2}$ is defined by (24), $\mathcal{Y}_{0}$ is defined by (23), and the operator $\mathcal{D}_{1}: \mathcal{X} \rightarrow \mathcal{Y}_{0}$ is defined by

$$
\mathcal{D}_{1}(\alpha, \hat{u}):=\left(\begin{array}{c}
* d \alpha+2 \pi \sum_{\nu=1}^{n}\left\langle u_{v}, \hat{u}_{v}\right\rangle \mathrm{w}_{v}-\operatorname{Re} \Psi(\hat{u}) \tau_{1} \\
d^{*} \alpha+2 \pi \sum_{\nu=1}^{n}\left\langle i u_{v}, \hat{u}_{\nu}\right\rangle \mathrm{w}_{v}-\operatorname{Im} \Psi(\hat{u}) \tau_{1} \\
\bar{\partial}_{A} \hat{u}_{v}+\rho_{\nu}(\alpha)^{0,1} u_{v}
\end{array}\right) .
$$

Since $\mathcal{L}$ is injective the determinant line of the complex (27) is that of the Fredholm operator $d \mathcal{S}^{\delta}: \mathcal{X}^{\delta} / \mathcal{L}($ Lie $\mathcal{G}) \rightarrow \mathcal{Z}_{0}$. Define $\mathcal{K}_{1}: \mathcal{X} \rightarrow \mathcal{V}_{0} \oplus \mathbb{R}^{2}$ by

$$
\mathcal{K}_{1}\left(\begin{array}{c}
\alpha \\
\hat{u}
\end{array}\right):=\left(\begin{array}{c}
d^{*} \alpha+2 \pi \sum_{v=1}^{n}\left\langle i u_{v}, \hat{u}_{v}\right\rangle \mathrm{w}_{v}-\operatorname{Im} \Psi(\hat{u}) \tau_{1} \\
\sum_{v \notin I} \int_{\Sigma}\left\langle u_{v}, \hat{u}_{\nu}\right\rangle \operatorname{dvol}_{\Sigma} \\
\operatorname{Im} \Psi(\hat{u})
\end{array}\right) .
$$

This map is surjective and there is an obvious isomorphism from the kernel of $\mathcal{K}_{1}$ to the quotient $\mathcal{X}^{\delta} / \mathcal{L}($ Lie $\mathcal{G})$. Hence Lemma B.4 provides an isomorphism from the determinant of the operator $d \mathcal{S}^{\delta}: \mathcal{X}^{\delta} / \mathcal{L}$ (Lie $\left.\mathcal{G}\right) \rightarrow \mathcal{Z}_{0}$ to that of the augmented operator $d \mathcal{S}^{\delta} \oplus \mathcal{K}_{1}: \mathcal{X} \rightarrow \mathcal{Y}_{0} \oplus \mathbb{R}^{2}=\mathcal{Z}_{0} \oplus \mathcal{V}_{0} \oplus \mathbb{R}^{2}$. Since the real part of the complex linear map $\Psi$ defined by (22) is the differential of the map $u \mapsto t$ given by (26), this augmented operator agrees with $\mathcal{D}_{1} \oplus \Phi$. Now $\mathcal{D}_{1}$ is complex linear and so, via Lemma B.1, the complex orientation of $\operatorname{det}\left(\mathcal{D}_{1}\right)$ determines an orientation of the $T$-moduli problem $\left(\mathcal{B}^{\delta}, \mathcal{E}^{\delta}, \mathcal{S}^{\delta}\right)$.

That this orientation of $\left(\mathcal{B}^{\delta}, \mathcal{E}^{\delta}, \mathcal{S}^{\delta}\right)$ is indeed the opposite of the boundary orientation is verified in Remark 4.4 below. With the orientations understood, the 
(Cobordism) axiom in Theorem A.4 implies that the wall crossing number is given by

$$
\Phi_{\lambda, g}^{\rho, \tau_{0}+\varepsilon \tau_{1}}(\alpha)-\Phi_{\lambda, g}^{\rho, \tau_{0}-\varepsilon \tau_{1}}(\alpha)=\int_{\mathcal{M}^{\delta} / T} \pi^{*} \alpha
$$

It remains to prove that the integrals over $\mathcal{M}^{\delta} / T$ and $\mathcal{P}_{0} / T$ agree. We prove this by a homotopy argument. Fix a real parameter $s \in[0,1]$ and replace the second equation in (25) by

$$
* F_{A}+\pi \sum_{\nu \in I}\left|u_{v}\right|^{2} \mathrm{w}_{v}-\frac{*_{\mathfrak{t}} \lambda(P)}{\operatorname{Vol}(\Sigma)}-\tau_{0}=s\left(t \tau_{1}-\pi \sum_{\nu \notin I}\left|u_{v}\right|^{2} \mathrm{w}_{v}\right) .
$$

This defines a homotopy of sections $\mathcal{S}_{s}^{\delta}: \mathcal{B}^{\delta} \rightarrow \mathcal{E}^{\delta}, 0 \leq s \leq 1$, with $\mathcal{S}_{1}^{\delta}=\mathcal{S}^{\delta}$ and

$$
\mathcal{S}_{0}^{\delta}(A, u)=\left(* F_{A}+\pi \sum_{v \in I}\left|u_{v}\right|^{2} \mathrm{w}_{v}-\frac{*_{\mathfrak{t}} \lambda(P)}{\operatorname{Vol}(\Sigma)}-\tau_{0}, \bar{\partial}_{A} u_{v}\right) .
$$

The zero sets of these sections form a compact subset of $[0,1] \times \mathcal{B}^{\delta}$. Following the above discussion we obtain an isomorphism from the determinant line of the moduli problem $\left(\mathcal{B}^{\delta}, \mathcal{E}^{\delta}, \mathcal{S}_{s}^{\delta}\right)$ at a tuple $(s, A, u)$ to the determinant line of the Fredholm operator

$$
\mathcal{D}_{s} \oplus \Phi: \mathcal{X} \longrightarrow \mathcal{Y}_{0} \oplus \mathbb{R}^{2}
$$

where the operator $\mathcal{D}_{s}: \mathcal{X} \rightarrow \mathcal{Y}_{0}$ is defined by

$$
\mathcal{D}_{s}\left(\begin{array}{l}
\alpha \\
\hat{u}
\end{array}\right):=\mathcal{D}_{0}\left(\begin{array}{c}
\alpha \\
\hat{u}
\end{array}\right)+s\left(\begin{array}{c}
2 \pi \sum_{v \notin I}\left\langle u_{v}, \hat{u}_{\nu}\right\rangle \mathrm{w}_{\nu}-\operatorname{Re} \Psi(\hat{u}) \tau_{1} \\
2 \pi \sum_{\nu \notin I}\left\langle i u_{v}, \hat{u}_{v}\right\rangle \mathrm{w}_{\nu}-\operatorname{Im} \Psi(\hat{u}) \tau_{1} \\
0
\end{array}\right) .
$$

Since $\mathcal{D}_{s}$ is complex linear the resulting orientation of the moduli problem $\left(\mathcal{B}^{\delta}, \mathcal{E}^{\delta}\right.$, $\left.\mathcal{S}_{s}^{\delta}\right)$ varies continuously in this homotopy. Moreover, there is an obvious orientation preserving morphism from $\left(\mathcal{B}^{\delta}, \mathcal{E}^{\delta}, \mathcal{S}_{0}^{\delta}\right)$ to the $T$-moduli problem $\left(\mathcal{B}, \mathcal{E}_{0}, \mathcal{S}_{0}\right)$ discussed before Proposition 4.1. Hence it follows from the (Cobordism) and (Functoriality) axioms in Theorem A.4 that the integrals over $\mathcal{M}^{\delta} / T$ and $\mathcal{P}_{0} / T$ agree, and so equation (20) follows from (29). This proves the proposition.

Remark 4.4 (Boundary orientation). We prove that the above orientation of $\left(\mathcal{B}^{\delta}\right.$, $\left.\mathcal{E}^{\delta}, \mathcal{S}^{\delta}\right)$ is the opposite of the boundary orientation. To see this, fix a boundary point $[A, u] \in \mathcal{M}^{\delta}$, let $\mathcal{D}: \mathcal{X} \rightarrow \mathcal{Y}$ be the operator (9), and define $\Phi_{1}: \mathcal{X} \rightarrow \mathbb{R}$ to be the first component of the linear map (24), i.e.,

$$
\Phi_{1}(\alpha, \hat{u}):=\sum_{\nu \notin I} \int_{\Sigma}\left\langle u_{\nu}, \hat{u}_{\nu}\right\rangle \mathrm{dvol}_{\Sigma}
$$


Assume first that the restriction of $\mathcal{D}$ to the kernel of $\Phi_{1}$ is surjective. Then $\mathcal{W}^{\delta}$ is a manifold near $[t, A, u]$ (with $t$ given by (26)) that is transverse to the boundary of $\mathcal{R}$. Moreover, the tangent space of $\mathcal{W}^{\delta}$ is the kernel of the operator $\widetilde{\mathcal{D}}: \mathbb{R} \oplus \mathcal{X} \rightarrow \mathcal{Y}$ given by

$$
\widetilde{\mathcal{D}}(\hat{t}, \alpha, \hat{u}):=\mathcal{D}(\alpha, \hat{u})-\left(\hat{t} \tau_{1}, 0,0\right) .
$$

Each element in the kernel of this operator satisfies $\hat{t}=\operatorname{Re} \Psi(\hat{u})$ (take the inner product of the first component with $e_{1}$ and integrate over $\Sigma$ ). A positively oriented basis of the tangent space then has the form $v_{0}, v_{1}, \ldots, v_{2 m}$, where $v_{0}=(\hat{t}, \alpha, \hat{u})$ with $\hat{t}=\operatorname{Re} \Psi(\hat{u})>0$, and the vectors $v_{1}, \ldots, v_{2 m}$ form a positively oriented basis of ker $\mathcal{D} \subset \operatorname{ker} \widetilde{\mathcal{D}}$. Throughout we shall fix three vectors $w_{0}, v_{1}, w_{1} \in \mathcal{X}$ such that

$$
\begin{array}{lll}
\Phi_{1}\left(w_{0}\right)=1, & \mathcal{D} w_{0}=(0,0,0), & \Psi\left(w_{0}\right)=0, \\
\Phi_{1}\left(v_{1}\right)=0, & \mathcal{D} v_{1}=\left(\tau_{1}, 0,0\right), & \Psi\left(v_{1}\right)=1, \\
\Phi_{1}\left(w_{1}\right)=0, & \mathcal{D} w_{1}=\left(0, \tau_{1}, 0\right), & \Psi\left(w_{1}\right)=i
\end{array}
$$

Note that $w_{0}$ is an inward pointing vector tangent to $\mathcal{W}^{\delta}$, that $v_{1}$ is a tangent vector to the boundary, and that $w_{1}$ does not belong to the kernel of $\widetilde{\mathcal{D}}$. The tangent space of $\mathcal{M}^{\delta}$ is isomorphic to the subspace $\operatorname{ker} \mathcal{D}_{1} \cap \operatorname{ker} \Phi \subset \operatorname{ker} \widetilde{\mathcal{D}}$. The vector $v_{1}$ belongs to this subspace and a complement of $\mathbb{R} v_{1}$ is the intersection $\operatorname{ker} \mathcal{D} \cap \operatorname{ker} \Phi_{1}$. Choose a basis $v_{2}, \ldots, v_{2 m}$ of $\operatorname{ker} \mathcal{D} \cap \operatorname{ker} \Phi_{1}$ such that the vectors $v_{1}, \ldots, v_{2 m}$ form a positive basis of ker $\mathcal{D}_{1} \cap \operatorname{ker} \Phi$ (with respect to the opposite boundary orientation). Then the vectors $w_{0}, v_{1}, \ldots, v_{2 m}$ form a positive basis of ker $\widetilde{\mathcal{D}}$. We claim that the vectors $w_{0}, w_{1}, v_{1}, \ldots, v_{2 m}$ form a positive basis of the kernel of $\mathcal{D}_{1}$ (and hence $v_{1}, \ldots, v_{2 m}$ form a positive basis of the tangent space of $\mathcal{M}^{\delta}$ with respect to the orientation introduced in the proof of Proposition 4.1). To prove the claim note that the vectors $w_{0}, v_{2}, \ldots, v_{2 m}$ form a basis of ker $\mathcal{D}$ and the vectors $v_{1}, w_{0}, v_{2}, \ldots, v_{2 m}$ form a negative basis of ker $\widetilde{\mathcal{D}}$. Since $\operatorname{Re} \Psi\left(v_{1}\right)>0$ it follows that the vectors $w_{0}, v_{2}, \ldots, v_{2 m}$ form a negative basis of ker $\mathcal{D}$. Since $v_{1}, w_{1} \in \operatorname{ker} \mathcal{D}_{1}$ satisfy $\Psi\left(v_{1}\right)=1$ and $\Psi\left(w_{1}\right)=i$ it follows that the vectors $v_{1}, w_{1}, w_{0}, v_{2}, \ldots, v_{2 m}$ form a negative basis of ker $\mathcal{D}_{1}$ and thus $w_{0}, w_{1}, v_{1}, v_{2}, \ldots, v_{2 m}$ form a positive basis as claimed.

If the restriction of $\mathcal{D}$ to the kernel of $\Phi_{1}$ is not onto one can homotop to a situation where this condition is satisfied and use the compatibility of all our isomorphisms with the local trivializations of the determinant line bundle, or one can argue as follows. We only sketch the main points. The elliptic complex associated to the $T$-moduli problem $(\mathcal{R}, \mathcal{F}, \mathcal{T})$ has the form

$$
0 \longrightarrow \mathfrak{t} \stackrel{\mathcal{L}}{\longrightarrow} \mathbb{R} \oplus \frac{\mathcal{X}}{\mathcal{L}\left(\text { Lie } \mathcal{G}_{0}\right)} \stackrel{d \mathcal{T}}{\longrightarrow} \mathcal{Z} \longrightarrow 0 .
$$

Lemma B.4 provides an isomorphism from the determinant of (31) to the determinant of the augmented operator $\widetilde{\mathcal{D}}: \mathbb{R} \oplus \mathcal{X} \rightarrow \mathcal{Y}$, defined above. The latter 
is canonically oriented by Lemma B.1 and the complex orientation of $\operatorname{det}(\mathcal{D})$. Next it is convenient to identify the kernel and cokernel of $\widetilde{\mathcal{D}}$ with the kernel and cokernel of the operator

$$
\mathcal{D}_{1} \oplus \operatorname{Im} \Psi: \mathcal{X} \rightarrow \mathcal{Y}_{0} \oplus \mathbb{R},
$$

where $\mathcal{D}_{1}$ is defined by (28). These identifications are obtained by the inclusion $\mathcal{X} \rightarrow \mathbb{R} \oplus \mathcal{X}:(\alpha, \hat{u}) \mapsto(\operatorname{Re} \Psi(\hat{u}), \alpha, \hat{u})$ and the decomposition $\mathcal{Y}$ $\cong \mathcal{Y}_{0} \oplus \mathbb{R}\left(\tau_{1}, 0,0\right) \oplus \mathbb{R}\left(0, \tau_{1}, 0\right)$ with $\mathcal{Y}_{0} \oplus \mathbb{R}$ corresponding to the first and last summands. If one uses Lemma B.1 and the complex orientation of $\operatorname{det}\left(\mathcal{D}_{1}\right)$ to orient $\operatorname{det}\left(\mathcal{D}_{1} \oplus \operatorname{Im} \Psi\right)$, then the isomorphism $\operatorname{det}\left(\mathcal{D}_{1} \oplus \operatorname{Im} \Psi\right) \rightarrow \operatorname{det}(\widetilde{\mathcal{D}})$ just described is orientation reversing. Now restrict the operator $\mathcal{D}_{1} \oplus \operatorname{Im} \Psi$ to the kernel of $\Phi_{1}$ to obtain the augmented operator for $\left(\mathcal{B}^{\delta}, \mathcal{E}^{\delta}, \mathcal{S}^{\delta}\right)$. Then the opposite boundary orientation translates, via Lemma B.4, to the orientation of $\operatorname{det}\left(\mathcal{D}_{1} \oplus \operatorname{Im} \Psi \oplus \Phi_{1}\right)$. By Lemma B.3 going over to $\operatorname{det}\left(\mathcal{D}_{1} \oplus \Phi\right)=$ $\operatorname{det}\left(\mathcal{D}_{1} \oplus \Phi_{1} \oplus \operatorname{Im} \Psi\right)$ involves another sign change which compensates the minus sign in the isomorphism $\operatorname{det}\left(\mathcal{D}_{1} \oplus \operatorname{Im} \Psi \oplus \Phi_{1}\right) \rightarrow \operatorname{det}\left(\widetilde{\mathcal{D}} \oplus \Phi_{1}\right)$. Thus the composition $\operatorname{det}\left(\mathcal{D}_{1} \oplus \Phi\right) \rightarrow \operatorname{det}\left(\widetilde{\mathcal{D}} \oplus \Phi_{1}\right)$ is orientation preserving. This means precisely that the above orientation of $\left(\mathcal{B}^{\delta}, \mathcal{E}^{\delta}, \mathcal{S}^{\delta}\right)$ is the opposite boundary orientation as claimed.

The moduli space $\mathcal{S}_{0}$ is the sphere bundle in the kernel bundle of a family of Cauchy-Riemann operators over $\mathcal{M}_{0}$. In Section 4.3 we explain a general equivariant localization formula for such kernel bundles. The relevant index computation uses the Atiyah-Singer index theorem for families and will be carried out in Section 4.4. The next section explains the necessary background about the equivariant Euler class.

\subsection{The equivariant Euler class}

We begin with some recollections about the equivariant Euler class (see [8] for details). Let $X$ be a compact oriented smooth manifold, $E \rightarrow X$ be an oriented real vector bundle of rank $k$, and $\mathrm{G}$ be a compact Lie group which acts on $X$ and $E$ by orientation preserving diffeomorphisms such that the projection is equivariant and the action is linear on the fibres. We shall think of the action of $\mathrm{G}$ on $X$ and $E$ as a right action and denote it by $(x, e) \mapsto\left(g^{*} x, g^{*} e\right)$ for $e \in E_{x}$. The corresponding covariant action will be denoted by $g_{*} x:=\left(g^{-1}\right)^{*} x$ and the infinitesimal (contravariant) action of $\xi \in \mathfrak{g}:=\operatorname{Lie}(\mathrm{G})$ by $\xi^{*} x \in T_{x} X$. An equivariant Thom form is a $d_{\mathrm{G}}$-closed equivariant differential form $\tau_{\mathrm{G}}(E) \in \Omega_{\mathrm{G}}^{k}(E)$ with compact support and fibre integral one. The equivariant Euler class $e_{\mathrm{G}}(E) \in H_{\mathrm{G}}^{k}(X)$ is the cohomology class of the pullback of an equivariant Thom form under the zero section. We will sometimes use the same notation for the Euler class and a form representing it. The Thom class and the Euler class are multiplicative under direct sum. 
Now suppose that $E$ is a rank $n$ complex vector bundle and the action of $\mathrm{G}$ is complex linear on the fibres. Then an explicit representative of the equivariant Euler class can be constructed as follows. Fix a G-invariant Hermitian metric on $E$ and let $P \rightarrow X$ denote the unitary frame bundle of $E$. This bundle carries a right action of $\mathrm{U}(n)$ and a left action of $\mathrm{G}$, and these two action commute. Let $X_{\xi} \in \operatorname{Vect}(P)$ denote the infinitesimal (covariant) action of $\xi \in \mathfrak{g}$. More precisely a point $p \in P_{x}$ of the fibre over $x \in X$ is a unitary vector space isomorphism $p: \mathbb{C}^{n} \rightarrow E_{x}$ and the left action of $g \in \mathrm{G}$ is given by $g_{*} p: \mathbb{C}^{n} \rightarrow E_{g_{*} x}$. The vector field $X_{\xi} \in \operatorname{Vect}(P)$ is defined by

$$
X_{\xi}(p):=\left.\frac{d}{d t}\right|_{t=0} \exp (t \xi)_{*} p \in T_{p} P .
$$

The following formula was established in Berline-Vergne [5] and, in a more general setting, in Berline-Getzler-Vergne [6]. In particular, the term $A\left(X_{\xi}\right)$ coincides with the generalized moment map in $[5,6]$. We include a proof for the sake of completeness.

Lemma 4.5. Let $A \in \mathcal{A}(P) \subset \Omega^{1}(P, \mathfrak{u}(n))$ be a $\mathrm{G}$-invariant $\mathrm{U}(n)$-connection form on $P$. Then the G-equivariant Euler class of a complex vector bundle $E$ is represented by the $d_{\mathrm{G}}$-closed form

$$
e_{\mathrm{G}}(E, \xi)=\operatorname{det}\left(\frac{i}{2 \pi} F_{A}+\frac{i}{2 \pi} A\left(X_{\xi}\right)\right),
$$

where $F_{A} \in \Omega^{2}(P, \mathfrak{u}(n))$ denotes the curvature of $A$.

Proof. The right hand side in (32) is invariant and horizontal for the $\mathrm{U}(n)$-action and thus descends to a G-equivariant form on $X$. It is easy to check that this form is $d_{\mathrm{G}}$-closed and hence represents an equivariant cohomology class.

To prove (32) we assume first that $E=X \times \mathbb{C}^{n}$ is a trivial bundle and $\rho$ : $\mathrm{G} \rightarrow \mathrm{U}(n)$ is a unitary representation of $\mathrm{G}$. The homomorphism $\rho$ defines the covariant action of $\mathrm{G}$ on $E$ and so

$$
g^{*}(x, z)=\left(g^{*} x, \rho(g)^{-1} z\right)
$$

for $x \in X, z \in \mathbb{C}^{n}$, and $g \in \mathrm{G}$. The frame bundle of $E$ is the product bundle $P:=X \times \mathrm{U}(n)$ and the formula

$$
A_{x, u}(v, u \eta):=\eta
$$

for $v \in T_{x} X, u \in \mathrm{U}(n)$ and $\eta \in \mathfrak{u}(n)$ defines a $\mathrm{U}(n)$-connection form $A \in$ $\Omega^{1}(P, \mathfrak{u}(n))$. This connection is G-invariant and flat. For $\xi \in \mathfrak{g}$ the vector field $X_{\xi} \in \operatorname{Vect}(P)$ is given by

$$
X_{\xi}(x, u)=(0, \dot{\rho}(\xi) u)
$$


and so

$$
A\left(X_{\xi}(x, u)\right)=u^{-1} \dot{\rho}(\xi) u, \quad \operatorname{det}\left(\frac{i}{2 \pi} A\left(X_{\xi}\right)\right)=\operatorname{det}\left(\frac{i}{2 \pi} \dot{\rho}(\xi)\right) .
$$

Now a Thom form on $E$ can be constructed as follows. For $k=0, \ldots, n$ let $\sigma_{k}: \mathfrak{u}(n) \rightarrow \Omega^{2 n-2 k}\left(\mathbb{C}^{n}\right)$ be a polynomial map of degree $k$. It is shown in [8, Lemma 5.5] that these polynomials can be chosen such that $\sigma_{0} \in \Omega^{2 n}\left(\mathbb{C}^{n}\right)$ is the standard volume form,

$$
\sigma_{n}(\eta)=\operatorname{det}(i \eta)
$$

and

$$
\iota\left(v_{\eta}\right) \sigma_{k}(\eta)=\lambda \wedge \sigma_{k+1}(\eta)
$$

for each $k$, where $\lambda \in \Omega^{1}\left(\mathbb{C}^{n}\right)$ is the differential of the function $z \mapsto|z|^{2} / 2$ and the vector field $v_{\eta} \in \operatorname{Vect}\left(\mathbb{C}^{n}\right)$ is defined by $v_{\eta}(z):=\eta z$ for $\eta \in \mathfrak{u}(n)$. Now choose functions $f_{k}:[0, \infty) \rightarrow[0, \infty)$ with compact support such that $f_{0}(s)=0$ for $s \leq \delta$ and $s \geq 1$ and

$$
f_{k}^{\prime}(s)+f_{k-1}(s)=0, \quad f_{k}(1)=0,
$$

and

$$
\int_{0}^{\infty} s^{k} f_{0}(s) d s=0, \quad \int_{0}^{\infty} s^{n-1} f_{0}(s) d s=\frac{1}{2^{n-1} \operatorname{Vol}\left(S^{2 n-1}\right)}
$$

for $0 \leq k \leq n-2$. Then

$$
f_{k}(s)=\frac{1}{(k-1) !} \int_{s}^{1}(t-s)^{k-1} f_{0}(t) d t
$$

and hence $f_{k}(s)=0$ for $s<\delta$ and $k<n$ and

$$
f_{n}(0)=\frac{1}{2^{n-1}(n-1) ! \operatorname{Vol}\left(S^{2 n-1}\right)}=\frac{1}{(2 \pi)^{n}} .
$$

Now a Thom form on $E=X \times \mathbb{C}^{n}$ is given by

$$
\tau(\xi)=\sum_{k=1}^{n} f_{k}\left(|z|^{2} / 2\right) \sigma_{k}(\dot{\rho}(\xi)) .
$$

Its pullback under the zero section is given by

$$
e_{\mathrm{G}}(E)=f_{n}(0) \sigma_{n}(\dot{\rho}(\xi))=\operatorname{det}\left(\frac{i}{2 \pi} \dot{\rho}(\xi)\right) .
$$

This proves the lemma in the case $E=X \times \mathbb{C}^{n}$. For general G-equivariant bundles $E \rightarrow X$ the result follows from the (Naturality) axiom for the Euler class and the fact that the pullback of $E$ under the projection $P \rightarrow E$ is isomorphic to the trivial bundle $P \times \mathbb{C}^{n}$. 
Remark 4.6. The formula of Lemma 4.5 can also be expressed as follows. Let $\nabla$ be a G-equivariant Hermitian connection on $E$ and, for $\xi \in \mathfrak{g}$, denote by $\xi^{\nabla} \in \Omega^{0}(X, \operatorname{End}(E))$ the covariant infinitesimal action defined by

$$
\xi^{\nabla} e:=\left.\nabla_{t} \exp (t \xi)_{*} e\right|_{t=0}
$$

Then the Euler class is given by

$$
e_{\mathrm{G}}(E, \xi)=\operatorname{det}\left(\frac{i}{2 \pi} F^{\nabla}+\frac{i}{2 \pi} \xi^{\nabla}\right) \text {. }
$$

Example 4.7. Let $E \rightarrow X$ be a rank $n$ complex vector bundle. Suppose $\mathrm{G}$ acts trivially on $X$ and that the covariant action on the fibres is given by a homomorphism $\rho: \mathrm{G} \rightarrow S^{1}$, given by

$$
\rho(\exp (\xi))=e^{-2 \pi i\langle\mathrm{w}, \xi\rangle},
$$

where $\mathrm{w} \in \mathfrak{g}^{*}$. Then, for every $\mathrm{G}$-invariant Hermitian connection $\nabla$ on $E$, the endomorphism $\xi^{\nabla} \in \Omega^{0}(X, \operatorname{End}(E))$ is given by multiplication with the imaginary number $\dot{\rho}(\xi)$. Hence

$$
e_{\mathrm{G}}(E, \xi)=\operatorname{det}\left(\frac{i}{2 \pi} F^{\nabla}+\frac{i}{2 \pi} \dot{\rho}(\xi)\right)=\sum_{j=0}^{n}\langle\mathrm{w}, \xi\rangle^{n-j} c_{j}(E) .
$$

We wish to invert the equivariant Euler class. This requires an extension of the equivariant cohomology ring of $X$.

Standing assumption. In the following $X$ is a smooth manifold, $\mathrm{G}$ is a compact Lie group acting on $X$, and $T_{1} \subset \mathrm{G}$ is an oriented circle which is contained in the center of $\mathrm{G}$ and acts trivially on $X$.

Denote the quotient group by $\mathrm{G}_{0}:=\mathrm{G} / T_{1}$. Denote by $\mathrm{e}_{1}$ the positive integral generator of the Lie algebra $\mathfrak{t}_{1}:=\operatorname{Lie}\left(T_{1}\right)$. Let $n$ be an integer. A $T_{1}$-rational G-equivariant differential form of degree $n$ on $X$ is a Laurent series in $z^{-1}$ of the form

$$
\alpha(\xi, z)=\sum_{j \leq n / 2} \alpha_{j}(\xi) z^{j}
$$

with coefficients $\alpha_{j} \in \Omega_{\mathrm{G}}^{n-2 j}(X)$, that satisfies the following conditions.

(i) For every $\xi \in \mathfrak{g}$ and every $x \in X$ the Laurent series $\sum_{j \leq n / 2} \alpha_{j}(\xi)_{x} z^{j}$ is a rational function on $\mathbb{C}$ with values in the complex vector space $\Lambda^{*} T_{x}^{*} X \otimes \mathbb{C}$.

(ii) For every $t \in \mathbb{R}$ we have $\alpha(\xi, t+z)=\alpha\left(\xi+t e_{1}, z\right)$. Equivalently,

$$
\begin{aligned}
& \alpha_{k}\left(\xi+t e_{1}\right)=\sum_{k \leq j \leq n / 2}\left(\begin{array}{l}
j \\
k
\end{array}\right) \alpha_{j}(\xi) t^{j-k}, \quad k \geq 0, \\
& \alpha_{k}\left(\xi+t e_{1}\right)=\sum_{k \leq j<0}\left(\begin{array}{l}
-k-1 \\
-j-1
\end{array}\right) \alpha_{j}(\xi)(-t)^{j-k}, \quad k<0 .
\end{aligned}
$$


Denote by $\Omega_{\mathrm{G}, T_{1}}^{n}(X)$ the space of $T_{1}$-rational G-equivariant differential forms on $X$. This is a chain complex with respect to the usual equivariant differential $d_{\mathrm{G}} \alpha(\xi):=d \alpha(\xi)+\iota\left(X_{\xi}\right) \alpha(\xi)$. The cohomology of this chain complex will be denoted by $H_{\mathrm{G}, T_{1}}^{*}(X)$.

Let $\alpha=\sum_{j} \alpha_{j} z^{j} \in \Omega_{\mathrm{G}, T_{1}}^{n}(X)$. Then $\alpha_{-1}\left(\xi+t e_{1}\right)=\alpha_{-1}(\xi)$. In other words, the coefficient of $z^{-1}$ descends to a $\mathrm{G}_{0}$-equivariant cohomology class on $X$. Minus this coefficient is called the residue at infinity of $\alpha$ and will be denoted by

$$
\operatorname{Res}_{\infty}(\alpha):=-\alpha_{-1}(\xi)=-\frac{1}{2 \pi i} \oint \alpha(\xi, z) d z \in \Omega_{\mathrm{G}_{0}}^{n+2}(X)
$$

The residue at infinity descends to a homomorphism

$$
\operatorname{Res}_{\infty}: H_{\mathrm{G}, T_{1}}^{n}(X) \rightarrow H_{\mathrm{G}_{0}}^{n+2}(X) .
$$

Remark 4.8. There is an obvious inclusion $\Omega_{\mathrm{G}}^{*}(X) \rightarrow \Omega_{\mathrm{G}, T_{1}}^{*}(X)$ whose image is the subspace of polynomials $\alpha=\sum_{0 \leq j \leq n / 2} \alpha_{j} z^{j} \in \Omega_{\mathrm{G}, T_{1}}^{n}(X)$. Condition (33) shows that any such form is uniquely determined by $\alpha_{0} \in \Omega_{\mathrm{G}}^{n}(X)$ and vice versa. The inclusion $\Omega_{\mathrm{G}}^{*}(X) \hookrightarrow \Omega_{\mathrm{G}, T_{1}}^{*}(X)$ induces an inclusion in cohomology

$$
H_{\mathrm{G}}^{*}(X) \hookrightarrow H_{\mathrm{G}, T_{1}}^{*}(X)
$$

whose left inverse is induced by the projection $\alpha=\sum_{j} \alpha_{j} z^{j} \mapsto \alpha_{0}$.

Let $E \rightarrow X$ be a G-equivariant complex vector bundle of rank $n_{E}$. The subgroup $T_{1}$ acts on $E$ with weight

$$
\mathrm{w}_{E}:=\operatorname{det}\left(\frac{i}{2 \pi} \dot{\rho}_{x}\left(e_{1}\right)\right) \in \mathbb{Z} .
$$

Here the homomorphism $\rho_{x}: T_{1} \rightarrow \operatorname{Aut}\left(E_{x}\right)$ denotes the action on the fiber over $x$ and $\dot{\rho}_{x}: \mathfrak{t}_{1} \rightarrow \operatorname{End}\left(E_{x}\right)$ denotes the corresponding Lie algebra homomorphism. The weight $\mathrm{w}_{E}$ is independent of $x$. Think of the equivariant Euler class as a polynomial map $\mathfrak{g} \rightarrow \Omega^{*}(X)$. By Lemma 4.5, the G-equivariant Chern classes $c_{j}(E) \in H_{\mathrm{G}}^{2 j}(X)$ are the coefficients of $z^{n_{E}-j}$ in the polynomial

$$
e_{\mathrm{G}}\left(E, \xi+z e_{1}\right)=: \sum_{j=0}^{n_{E}} c_{j}(E, \xi) z^{n_{E}-j}
$$

In particular, $c_{0}(E, \xi)=\mathrm{w}_{E}$. If $\mathrm{w}_{E} \neq 0$ then the equivariant Euler class $e_{\mathrm{G}}$ $\in H_{T}^{2 n_{E}}(X)$ has a well defined inverse $1 / e_{\mathrm{G}}$ in the $T_{1}$-rational G-equivariant cohomology group $H_{\mathrm{G}, T_{1}}^{-2 n_{E}}(X)$. To see this, expand the rational function $z \mapsto 1 / e_{\mathrm{G}}$ 
$\left(\xi+z e_{1}\right)$ into a Laurent series in $z^{-1}$ which converges near infinity:

$$
\begin{aligned}
\frac{1}{e_{\mathrm{G}}\left(E, \xi+z e_{1}\right)} & =\frac{1}{\mathrm{w}_{E} z^{n_{E}}} \sum_{k=0}^{\infty}\left(-\sum_{j=1}^{n_{E}} \frac{c_{j}(E, \xi)}{\mathrm{w}_{E}} z^{-j}\right)^{k} \\
& =: \sum_{i=0}^{\infty} s_{i}(E, \xi) z^{-n_{E}-i} .
\end{aligned}
$$

The coefficients $s_{i}(E) \in H_{\mathrm{G}}^{2 i}(X)$ of this Laurent series are called the equivariant Segre classes of $E$. They are uniquely determined by the equation

$$
\sum_{i+j=k} s_{i}(E, \xi) c_{j}(E, \xi)=\left\{\begin{array}{l}
1, \text { if } k=0 \\
0, \text { if } k>0
\end{array}\right.
$$

In particular, the degree zero Segre class is $s_{0}(E, \xi)=1 / \mathrm{w}_{E}$. If $F \rightarrow X$ is another G-equivariant complex vector bundle of rank $n_{F}$ with weight $\mathrm{w}_{F}$, then the quotient

$$
e_{\mathrm{G}}(F \ominus E):=\frac{e_{\mathrm{G}}(F)}{e_{\mathrm{G}}(E)} \in H_{\mathrm{G}, T_{1}}^{2 n_{F}-2 n_{E}}(X)
$$

depends only on the equivariant $K$-theory class $F \ominus E \in K_{\mathrm{G}}(X)$. It is only defined for equivariant $K$-theory classes $F \ominus E$ whose denominator $E$ has nonzero weight.

\subsection{Localization}

Let $X$ be an orientable smooth manifold, $\mathrm{G}=T$ be a torus acting on $X$, and $T_{1} \subset T$ be an oriented circle that acts trivially on $X$. We assume that the quotient group $T_{0}:=T / T_{1}$ acts on $X$ with finite isotropy. Denote the Lie algebras by $\mathfrak{t}:=\operatorname{Lie}(T), \mathfrak{t}_{1}:=\operatorname{Lie}\left(T_{1}\right)$, and $\mathfrak{t}_{0}:=\mathfrak{t} / \mathfrak{t}_{1}:=\operatorname{Lie}\left(T_{0}\right)$, let $\Lambda \subset \mathfrak{t}$ be the integer lattice, and denote by $e_{1} \in \mathfrak{t}_{1} \cap \Lambda$ the positive generator of the sublattice. Throughout we denote $m:=\operatorname{dim} X-\operatorname{dim} T_{0}$.

Let $\mathcal{E} \rightarrow X$ and $\mathcal{F} \rightarrow X$ be complex Hilbert space bundles on which $T$ acts complex linearly such that the projections are equivariant. Assume that $T_{1}$ acts with finite isotropy outside the zero sections of $\mathcal{E}$ and $\mathcal{F}$. Let

$$
\mathcal{D}_{x}: \mathcal{E}_{x} \rightarrow \mathcal{F}_{x}
$$

be a smooth family of G-equivariant complex linear Fredholm operators of complex numerical index

$$
\operatorname{index}(\mathcal{D}):=\operatorname{dim}^{\mathbb{C}} \operatorname{ker} \mathcal{D}_{x}-\operatorname{dim}^{\mathbb{C}} \operatorname{coker} \mathcal{D}_{x} .
$$


Denote by

$$
\mathcal{I N D}(\mathcal{D}):=\bigcup_{x \in X}\{x\} \times \operatorname{ker} D_{x} \ominus \operatorname{coker} \mathcal{D}_{x} \in K_{\mathrm{G}}(X)
$$

the topological index of $\mathcal{D}$, understood as a G-equivariant $K$-theory class. Consider the following $\mathrm{G}$-moduli problem. The Hilbert manifold $\mathbb{B}$ is given by

$$
\mathbb{B}:=\left\{(x, e) \mid x \in X, e \in \mathcal{E}_{x},\|e\|^{2}=1\right\},
$$

the Hilbert space bundle $\mathbb{H} \rightarrow \mathbb{B}$ has fibre

$$
\mathbb{H}_{x, e}:=\mathcal{F}_{x}
$$

over $(x, e) \in \mathbb{B}$, and the section $\mathbb{S}: \mathbb{B} \rightarrow \mathbb{H}$ is given by

$$
\mathbb{S}(x, e):=\mathcal{D}_{x} e .
$$

The zero set of this section is the kernel manifold

$$
\mathbb{M}:=\left\{(x, e) \in \mathbb{B} \mid D_{x} e=0\right\} .
$$

Denote by $\pi: \mathbb{B} \rightarrow X$ the obvious projection. The equivariant $K$-theory class $\mathcal{I N D}(\mathcal{D}) \in K_{\mathrm{G}}(X)$ has a nonzero weight (for the $T_{1}$-action) and hence carries an equivariant Euler class

$$
e_{\mathrm{G}}(\mathcal{I N D}(\mathcal{D})) \in H_{T, T_{1}}^{*}(X) .
$$

in the $T_{1}$-rational $T$-equivariant cohomology of $X$. The following theorem generalizes the localization formula for circle actions in [8]. The assertion requires a choice of orientations.

Remark 4.9. Orientations of $X / T_{0}$ and $T_{1}$ determine an orientation of the $T$-moduli problem $(\mathbb{B}, \mathbb{H}, \mathbb{S})$ as follows. By choosing local trivializations we may assume that $\mathcal{E}$ and $\mathcal{F}$ are (complex) Hilbert spaces equipped with a $T$-action and so $\mathcal{D}$ is a $T$-equivariant smooth map $X \rightarrow \mathcal{L}(\mathcal{E}, \mathcal{F}): x \mapsto \mathcal{D}_{x}$, which assigns a (complex linear) Fredholm operator $\mathcal{D}_{x}$ to every $x \in X$. In this case the vertical differential of $\mathbb{S}$ at a point $(x, e) \in \mathbb{M}$ is an operator

$$
D \mathbb{S}(x, e):\left\{(\hat{x}, \hat{e}) \in T_{x} X \times \mathcal{E} \mid\langle\hat{e}, e\rangle=0\right\} \rightarrow \mathcal{F} .
$$

It is given by

$$
D \mathbb{S}(x, e)(\hat{x}, \hat{e})=\mathcal{D}_{x} \hat{e}+\dot{\mathcal{D}}(\hat{x}) e,
$$

where $\dot{D}(\hat{x}) e$ is defined as the derivative of the path $\mathbb{R} \rightarrow \mathcal{F}: t \mapsto \mathcal{D}_{\exp _{x}(t \hat{x})} e$ at $t=0$. Now suppose that $\mathcal{D}_{x}$ is surjective. Then a positive basis of the kernel of $D \mathbb{S}(x, e)$ is defined as follows. Pick a positive basis $\hat{x}_{1}, \ldots, \hat{x}_{m}$ of $T_{x} X / \mathfrak{t}_{0} x$ 
and choose $\hat{e}_{1}, \ldots, \hat{e}_{m} \in \mathcal{E}$ such that $\mathcal{D}_{x} \hat{e}_{i}+\dot{\mathcal{D}}\left(\hat{x}_{i}\right) e=0$ and $\left\langle\hat{e}_{i}, e\right\rangle=0$ for $i=1, \ldots, m$. Next choose a positive basis $\hat{e}_{m+1}, \ldots, \hat{e}_{m+2 n}$ of the complex vector space ker $\mathcal{D}_{x}$ such that $\hat{e}_{m+2 n-1}$ is a positive tangent vector of the $T_{1}$-orbit of $e$ and $\hat{e}_{m+2 n}=e$.

Then the vectors $\left(\hat{x}_{i}, \hat{e}_{i}\right)$ for $i=1, \ldots, m$ and the vectors $\left(0, \hat{e}_{j}\right)$ for $j$ $=m+1, \ldots, m+2 n-2$ are declared to be a positive basis of ker $D \mathbb{S}(x, e) / \mathfrak{t}$. $(x, e)$. This definition of the orientation is independent of the choices. If $\mathcal{D}_{x}$ is not surjective, one can apply the same construction to the kernel of a suitably augmented operator. We emphasize that the orientation described here agrees with the convention of Remark 4.2.

Theorem 4.10. Let $m:=\operatorname{dim} X-\operatorname{dim} T_{0}$ and $n:=\operatorname{index}(\mathcal{D})$. Fix any orientation of $X / T_{0}$, let $T_{1}$ be oriented by $e_{1}$, and orient $\mathbb{M} / T$ as in Remark 4.9. Then

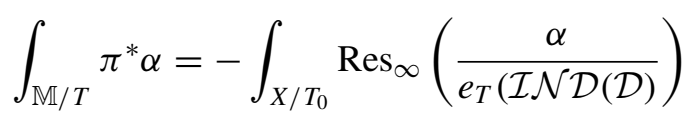

for every $\alpha \in H_{T}^{m+2 n-2}(X)$.

The integral on the left is understood as the Euler class of the $T$-moduli problem $\left(\mathbb{B}, \mathbb{H}, \mathbb{S}\right.$ ) evaluated on $\pi^{*} \alpha$ (see [8]). The integrand on the right is the residue at infinity of the $T_{1}$-rational $T$-equivariant cohomology class $\alpha / e_{T}(\mathcal{I N D}(\mathcal{D})) \in$ $H_{T, T_{1}}^{m-2}(X)$. It is a $T_{0}$ equivariant cohomology class in $H_{T_{0}}^{m}(X)$ and can be integrated over $X / T_{0}$ because $T_{0}$ acts on $X$ with finite isotropy.

Proof of Theorem 4.10. The proof has three steps.

Step 1. We may assume without loss of generality that $\mathcal{E}$ is finite dimensional and admits an equivariant trivialization and that $\mathcal{F}=0$.

The reduction to the finite dimensional case is proved as in [8, Theorem 11.1]. Hence assume $E=\mathcal{E}$ and $F=\mathcal{F}$ are finite dimensional. By Proposition C.1, there exists a $T$-equivariant complex vector bundle $E^{\prime} \rightarrow X$ such that $E \oplus E^{\prime}$ is equivariantly isomorphic to $X \times V$ for some complex $T$-representation $V$. Since $T$ is a torus there exists a homomorphism $T \rightarrow S^{1}$ whose restriction to $T_{1}$ has nonzero degree. Multiplying the action of $T$ on $E^{\prime}$ with a suitable power of this homomorphism we may assume that the action of $T_{1}$ on $E^{\prime}$ has nonzero weight. Now let $\mathbb{B}^{\prime} \subset E \oplus E^{\prime}$ be the unit sphere bundle, $\mathbb{H}^{\prime} \rightarrow \mathbb{B}^{\prime}$ be the pullback of $F \oplus E^{\prime}$ under the projection $\pi^{\prime}: \mathbb{B}^{\prime} \rightarrow X$, and $\mathbb{S}^{\prime}: \mathbb{B}^{\prime} \rightarrow \mathbb{H}^{\prime}$ be given by

$$
\mathbb{S}^{\prime}\left(x, e, e^{\prime}\right):=\left(x, D_{x} e, e^{\prime}\right) .
$$

Then the inclusion $\mathbb{B} \rightarrow \mathbb{B}^{\prime}:(x, e) \mapsto(x, e, 0)$ defines a morphism of $T$-moduli problems. Hence, assuming the assertion for $E$ replaced by $X \times V$ and $F$ replaced 
by the zero bundle, we obtain

$$
\begin{aligned}
\int_{\mathbb{M} / T} \pi^{*} \alpha & =\chi^{\mathbb{B}, \mathbb{H}, \mathbb{S}^{*}}\left(\pi^{*} \alpha\right) \\
& =\chi^{\mathbb{B}^{\prime}, \mathbb{H}^{\prime}, \mathbb{S}^{\prime}}\left(\pi^{\prime *} \alpha\right) \\
& =\int_{\mathbb{B}^{\prime} / T} \pi^{\prime *} \alpha \wedge e_{T}\left(\mathbb{H}^{\prime}\right) \\
& =\int_{\mathbb{B}^{\prime} / T} \pi^{\prime *} \alpha \wedge \pi^{\prime *} e_{T}\left(F \oplus E^{\prime}\right) \\
& =-\int_{X / T_{0}} \operatorname{Res}_{\infty}\left(\frac{\alpha \wedge e_{T}\left(F \oplus E^{\prime}\right)}{e_{T}\left(E \oplus E^{\prime}\right)}\right) \\
& =-\int_{X / T_{0}} \operatorname{Res}_{\infty}\left(\frac{\alpha}{e_{T}(\mathcal{I} \mathcal{N D}(D))}\right) .
\end{aligned}
$$

Here the second equation uses the (Functoriality) axiom for the Euler class, the third equation uses the (Thom class) axiom (see Appendix A), the fourth equation uses the fact that $\mathbb{H}^{\prime}$ is the pullback of $F \oplus E^{\prime}$, and the fifth equation uses the hypothesis that the result holds when $E$ is a trivial bundle and $F=0$.

Step 2. Suppose $E=L=X \times \mathbb{C}$ is a trivial line bundle and denote by $B \subset L$ the unit circle bundle. Then for every $\alpha=\sum_{j \leq m / 2} \alpha_{j} z^{j} \in \Omega_{T, T_{1}}^{m}(X)$,

$$
\int_{B / T} \pi^{*} \alpha_{0}=-\int_{X / T_{0}} \operatorname{Res}_{\infty}\left(\frac{\alpha}{e_{T}(L)}\right) .
$$

Let $\rho: T \rightarrow S^{1}$ denote the covariant action of $T$ on the fibres of $L$ and suppose that $T_{1}$ acts on the fibers with weight $\ell$. Then $\dot{\rho}\left(e_{1}\right)=-2 \pi i \ell$ and, by Lemma 4.5,

$$
e_{T}\left(L, \xi+z e_{1}\right)=\ell z+\frac{i \dot{\rho}(\xi)}{2 \pi} .
$$

Each form $\alpha_{j} \in \Omega_{T}^{m-2 j}(X)$ is equivariantly closed and hence represents a $T$-equivariant cohomology class on $X$. Now

$$
\begin{aligned}
\frac{\alpha(\xi, z)}{e_{T}\left(\xi+z e_{1}\right)} & =\left(\sum_{j \leq m / 2} \frac{\alpha_{j}(\xi)}{\ell} z^{j-1}\right) \frac{1}{1+\frac{i \dot{\rho}(\xi)}{2 \pi \ell z}} \\
& =\left(\sum_{j \leq m / 2} \frac{\alpha_{j}(\xi)}{\ell} z^{j-1}\right) \sum_{k \geq 0}\left(-\frac{i \dot{\rho}(\xi)}{2 \pi \ell z}\right)^{k} .
\end{aligned}
$$

The residue at infinity is minus the coefficient of $z^{-1}$ in this power series. Thus

$$
\operatorname{Res}_{\infty}\left(\frac{\alpha}{e_{T}(L)}\right)=-\frac{1}{\ell} \sum_{j \geq 0} \alpha_{j}(\xi)\left(-\frac{i \dot{\rho}(\xi)}{2 \pi \ell}\right)^{j}
$$


By (33), the right hand side is invariant under the shift $\xi \mapsto \xi+t e_{1}$ and hence descends to a $T_{0}$-equivariant differential form on $X$.

To compute the integral of $\pi^{*} \alpha_{0}$ over $B / T$, we denote the elements of $B$ $=X \times S^{1}$ by $(x, u)$, where $x \in X$ and $u \in S^{1}$. Then $u^{-1} d u$ is the standard $\mathrm{U}(1)$-connection form on $B$. Define $A_{1} \in \Omega^{1}\left(B, \mathfrak{t}_{1}\right)$ by

$$
A_{1}:=-\frac{i u^{-1} d u}{2 \pi \ell} e_{1} \text {. }
$$

This form is $T$-invariant and satisfies $A_{1}\left(0,-\dot{\rho}\left(e_{1}\right) z\right)=\left(i \dot{\rho}\left(e_{1}\right) / 2 \pi \ell\right) e_{1}=e_{1}$. Hence it is a $T$-invariant $T_{1}$-connection as in [8], where we regard the action by $\rho^{-1}$ as the contravariant action on $B$. The infinitesimal covariant action of $\xi \in \mathfrak{t}$ on $B$ is given by $X_{\xi}(x, u)=(0, \dot{\rho}(\xi) u)$. Hence the $T$-equivariant curvature of $A_{1}$ is the 2 -form $F_{A_{1}, T} \in \Omega_{T}^{2}(B, \mathfrak{t})$ given by

$$
F_{A_{1}, T}(\xi):=F_{A_{1}}+\xi+A_{1}\left(X_{\xi}\right)=\xi-\frac{i \dot{\rho}(\xi)}{2 \pi \ell} e_{1} .
$$

(see [8, Section 3]). Replacing $\xi$ by $F_{A_{1}, T}(\xi)$ in the equivariant differential form $\pi^{*} \alpha_{0} \in \Omega_{T}^{m}(B)$ we obtain the $T_{1}$-basic $T$-equivariant differential form

$$
\left(\pi^{*} \alpha_{0}\right)_{A_{1}}=\pi^{*} \alpha_{0}\left(\xi-\frac{i \dot{\rho}(\xi)}{2 \pi \ell} e_{1}\right)=\sum_{j \geq 0} \pi^{*} \alpha_{j}(\xi)\left(-\frac{i \dot{\rho}(\xi)}{2 \pi \ell}\right)^{j}
$$

on $B=X \times S^{1}$. The projection $\pi: B \rightarrow X$ induces a $T_{0}$-equivariant diffeomorphism from $B / T_{1}$ to $X$, however, each point in $B$ has an isotropy subgroup of order $\ell$ under the action of $T_{1}$. Moreover, the diffeomorphism is orientation preserving if and only if $\ell$ is positive. (If $\xi_{1}, \ldots, \xi_{m}$ is a positive basis of $T_{x} X / T_{0}$ and $u \in S^{1}$ then, according to Remark 4.9 , the basis $\left(\xi_{1}, 0\right), \ldots,\left(\xi_{m}, 0\right)$ of $T_{(x, u)} B / T$ is positive if and only if the vectors $\left(\xi_{1}, 0\right), \ldots,\left(\xi_{m}, 0\right),\left(0, \dot{\rho}\left(e_{1}\right) u\right),(0, u)$ form a positive basis of $T_{x} X / T_{0} \times \mathbb{C}$. Since $\dot{\rho}\left(e_{1}\right)=-2 \pi i \ell$, this is the case if and only if $\ell$ is positive.) Hence

$$
\int_{B / T} \pi^{*} \alpha_{0}=\int_{B / T}\left(\pi^{*} \alpha_{0}\right)_{A_{1}}=\frac{1}{\ell} \sum_{j \geq 0} \int_{X / T_{0}} \alpha_{j}\left(\xi_{0}\right)\left(-\frac{i \dot{\rho}\left(\xi_{0}\right)}{2 \pi \ell}\right)^{j},
$$

and so the assertion of Step 2 follows from (38).

Step 3. We prove the theorem.

By Step 1, we may assume without loss of generality that $F=0$ and $E=X \times V$ for some unitary $T$-representation $V$. Since $T$ is a torus, we may assume that $V=\mathbb{C}^{n}$ and that $T$ acts diagonally by homomorphisms $\rho_{\nu}: T \rightarrow S^{1}$ for $v=1, \ldots, n$. Denote by $L_{v}:=X \times \mathbb{C}$ the $T$-equivariant bundle where $T$ acts by $\rho_{\nu}$ on the fibre. Consider the $T$-moduli problem $(B, H, S)$ given by

$$
B:=X \times S^{2 n-1}, \quad H:=\pi^{*} L_{1} \oplus \cdots \oplus \pi^{*} L_{n-1},
$$


where $\pi: B \rightarrow X$ denotes the projection, and

$$
S\left(x, z_{1}, \ldots, z_{n}\right):=\left(z_{1}, \ldots, z_{n-1}\right) .
$$

Then the $T$-equivariant Euler class of $H$ is the pullback under $\pi$ of the Euler class of $L_{1} \oplus \cdots \oplus L_{n-1}$, i.e.

$$
e_{T}(H)=\pi^{*} e_{T}\left(L_{1}\right) \wedge \cdots \wedge \pi^{*} e_{T}\left(L_{n-1}\right) .
$$

Let $\alpha \in \Omega_{T}^{m+2 n-2}(X)$ and define $\beta \in \Omega_{T, T_{1}}^{m}(X)$ by

$$
\beta:=\sum_{j \leq m / 2} \beta_{j} z^{j}:=\frac{\alpha}{e_{T}\left(L_{1}\right) \cdots e_{T}\left(L_{n-1}\right)}, \quad \beta_{j} \in \Omega_{T}^{m-2 j}(X) .
$$

Since $T$ acts on $B$ with finite isotropy, we can represent the equivariant cohomology class $e_{T}(H) \in H_{T}^{2 n-2}(B)$ by a $T$-invariant and horizontal differential form $\tau_{H} \in \Omega^{2 n-2}(B)$ (see [8, Theorem 3.8]). With such a representative the identity $\pi^{*} \alpha=\pi^{*} \beta \wedge e_{T}(H)$ in $\Omega_{T, T_{1}}^{*}(X)$ takes the form $\pi^{*} \alpha=\pi^{*} \beta_{0} \wedge \tau_{H}$. Now $S$ is transverse to the zero section and $S^{-1}(0)$ is the unit sphere bundle $B_{n}:=B \cap L_{n}$ in $L_{n}$. Hence it follows from the (Transversality) axiom for the Euler class in Appendix A that

$$
\int_{B / T} \pi^{*} \alpha=\int_{B / T} \pi^{*} \beta_{0} \wedge \tau_{H}=\int_{B_{n} / T} \pi^{*} \beta_{0}=-\int_{X / T_{0}} \operatorname{Res}_{\infty}\left(\frac{\beta}{e_{T}\left(L_{n}\right)}\right) .
$$

The last equation follows from Step 2. Since $\beta / e_{T}\left(L_{n}\right)=\alpha / e_{T}(E)$, this proves Theorem 4.10.

Remark 4.11. Theorem 4.10 continues to hold if we replace $X$ by a $T_{0}$-moduli problem $\left(\mathcal{B}_{0}, \mathcal{E}_{0}, \mathcal{S}_{0}\right)$ as in Appendix $\mathrm{A}$ and $\mathcal{E}$ and $\mathcal{F}$ by Hilbert space bundles over $\mathcal{B}_{0}$. Then $\mathbb{B}$ is the unit sphere bundle in $\mathcal{E}, \mathbb{H}_{b, e}=\mathcal{E}_{0 b} \oplus \mathcal{F}_{b}, \mathbb{S}(b, e)=$ $\left(\mathcal{S}_{0}(b), \mathcal{D}_{b} e\right)$, and the right hand side of (36) is understood in terms of the Euler class of $\left(\mathcal{B}_{0}, \mathcal{E}_{0}, \mathcal{S}_{0}\right)$. To prove this, choose a finite dimensional reduction of $\left(\mathcal{B}_{0}, \mathcal{E}_{0}, \mathcal{S}_{0}\right)$ and note that (36) continues to hold for noncompact manifolds $X$ and compactly supported $T$-equivariant differential forms $\alpha$.

\subsection{The index formula}

We return to the setting of Section 3. Recall that $P \rightarrow \Sigma$ is a principal $T$-bundle and $L_{v}=P \times \rho_{\rho_{v}} \mathbb{C} \rightarrow \Sigma$ for $v=1, \ldots, n$. Given an index set $I \subset\{1, \ldots, n\}$ as in Section 3 we consider the principal $\mathcal{G}_{0}$-bundle

$$
\mathcal{P}_{I}:=\mathcal{A}(P) \times \bigoplus_{v \in I} \Omega^{0}\left(\Sigma, L_{v}\right) \rightarrow \mathcal{B}_{I}:=\mathcal{P}_{I} / \mathcal{G}_{0}
$$


where the based gauge group $\mathcal{G}_{0}$ acts by

$$
g^{*}\left(A, u_{v}\right)=\left(A+g^{-1} d g, \rho_{\nu}(g)^{-1} u_{v}\right) .
$$

It also acts on $L_{v_{0}}$ by

$$
g^{*}[p, \zeta]:=\left[p g(z)^{-1}, \zeta\right]=\left[p, \rho_{\nu_{0}}(g(z))^{-1} \zeta\right],
$$

where $z:=\pi(p) \in \Sigma$. For $v_{0} \notin I$ we consider the universal line bundle

$$
\mathbb{L}^{v_{0}}:=\frac{\mathcal{P}_{I} \times L_{\nu_{0}}}{\mathcal{G}_{0}} \rightarrow \mathcal{B}_{I} \times \Sigma .
$$

The torus $T$ acts on $\mathbb{L}^{\nu_{0}}$ by

$$
h^{*}\left[A, u_{v}, p, \zeta\right]:=\left[A, \rho_{v}(h)^{-1} u_{v}, p, \rho_{\nu_{0}}(h)^{-1} \zeta\right] .
$$

For $\mathbf{x}=[A, u] \in \mathcal{B}_{I}$ let us denote by $\mathbb{L}_{\mathbf{x}}^{v}$ the restriction of $\mathbb{L}^{v}$ to $\{\mathbf{x}\} \times \Sigma$. This restriction is equipped with a natural connection (induced by $A$ ) and hence with a Cauchy-Riemann operator

$$
\bar{\partial}_{\mathbf{x}}^{v}: \Omega^{0}\left(\Sigma, \mathbb{L}_{\mathbf{x}}^{v}\right) \rightarrow \Omega^{0,1}\left(\Sigma, \mathbb{L}_{\mathbf{x}}^{\nu}\right) .
$$

Next consider the universal vector bundle

$$
\mathbb{E}:=\bigoplus_{\nu \notin I} \mathbb{L}^{v}
$$

and its restrictions $\mathbb{E}_{\mathbf{x}}$ to $\{\mathbf{x}\} \times \Sigma$. The Cauchy-Riemann operators

$$
\bar{\partial}_{\mathbf{x}}: \Omega^{0}\left(\Sigma, \mathbb{E}_{\mathbf{x}}\right) \rightarrow \Omega^{0,1}\left(\Sigma, \mathbb{E}_{\mathbf{x}}\right)
$$

form a family of Fredholm operators over $\mathcal{B}_{I}$ between appropriate Hilbert space completions $\mathcal{E}_{\mathbf{x}}$ of $\Omega^{0}\left(\Sigma, \mathbb{E}_{\mathbf{x}}\right)$ and $\mathcal{F}_{\mathbf{X}}$ of $\Omega^{0,1}\left(\Sigma, \mathbb{E}_{\mathbf{x}}\right)$. These operators are complex linear and equivariant with respect to the action of $T$.

As in Section 3 we denote by $T_{1}$ the identity component of the isotropy subgroup of the subspace $\mathbb{C}^{I}:=\left\{x \in \mathbb{C}^{n} \mid x_{v}=0\right.$ for $\left.v \notin I\right\}$ and assume that $T_{1}$ is a circle. This circle acts trivially on the base $\mathcal{B}_{I}$ and with finite isotropy outside of the zero sections of $\mathcal{E}$ and $\mathcal{F}$. The quotient group $T_{0}:=T / T_{1}$ acts with finite isotropy on the moduli space $\mathcal{M}_{0} \subset \mathcal{B}_{I}$ of solutions of equation (13). Hence we are in the situation of Theorem 4.10. The relevant dimensions are

$$
\begin{aligned}
\operatorname{dim} \mathcal{M}-\operatorname{dim} T & =(n-\operatorname{dim} T)(2-2 g)+2 \sum_{\nu=1}^{n} d_{\nu}=: 2 m, \\
\operatorname{dim} \mathcal{M}_{0}-\operatorname{dim} T_{0} & =\left(|I|-\operatorname{dim} T_{0}\right)(2-2 g)+2 \sum_{\nu \in I} d_{\nu}+2 g, \\
\operatorname{index}(\bar{\partial}) & =\sum_{\nu \notin I}\left(d_{\nu}+1-g\right),
\end{aligned}
$$


where $d_{v}$ denotes the first Chern number of the bundle $L_{v} \rightarrow \Sigma$. Note that the kernel manifold of $\bar{\partial}$ is precisely the space $\mathcal{S}_{0}$ of solutions of equations (17). Hence, by Theorem 4.10 and Remark 4.11, we have

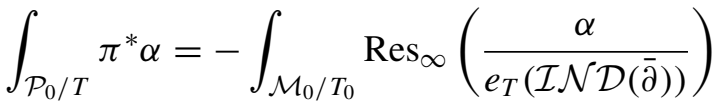

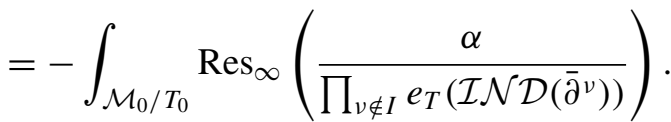

Theorem 3.1 now follows from

Lemma 4.12. Denote by $\pi_{0}^{*}: H_{T, T_{1}}^{*}\left(\mathcal{A} / \mathcal{G}_{0}\right) \rightarrow H_{T, T_{1}}^{*}\left(\mathcal{B}_{I}\right)$ the homomorphism induced by the projection $\pi_{0}: \mathcal{B}_{I} \rightarrow \mathcal{A} / \mathcal{G}_{0}$. Then, for every $v \notin I$,

$$
e_{T}\left(\mathcal{I N D}\left(\bar{\partial}^{\nu}\right)\right)=\pi_{0}^{*}\left\langle\mathrm{w}_{\nu}, \xi\right\rangle^{d_{\nu}+1-g} \exp \left(-\frac{\Omega_{\nu}}{\left\langle\mathrm{w}_{\nu}, \xi\right\rangle}\right) \in H_{T, T_{1}}^{*}\left(\mathcal{B}_{I}\right) .
$$

Proof of Theorem 3.1. By Proposition 4.1 and (39) we have

$$
\begin{aligned}
& \Phi_{\lambda, g}^{\rho, \tau_{0}+\varepsilon \tau_{1}}(\alpha)-\Phi_{\lambda, g}^{\rho, \tau_{0}-\varepsilon \tau_{1}}(\alpha)=\int_{\mathcal{P}_{0} / T} \pi^{*} \alpha
\end{aligned}
$$

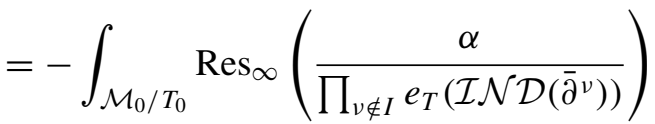

$$
\begin{aligned}
& =\int_{\mathcal{M}_{0} / T_{0}} \pi_{0}^{*} \alpha_{0} \text {, }
\end{aligned}
$$

where

$$
\alpha_{0}(\xi)=\frac{1}{2 \pi i} \oint \frac{\alpha\left(\xi+z e_{1}\right)}{\prod_{\nu \notin I}\left\langle\mathrm{w}_{\nu}, \xi+z e_{1}\right\rangle^{d_{\nu}+1-g}} \exp \left(\sum_{\nu \notin I} \frac{\Omega_{v}}{\left\langle\mathrm{w}_{\nu}, \xi+z e_{1}\right\rangle}\right) d z
$$

(by Lemma 4.12). This proves Theorem 3.1.

Proof of Lemma 4.12. Note first that $\mathbb{L}^{v}$ is the pullback under the projection $\pi_{0} \times$ id : $\mathcal{B}_{I} \times \Sigma \rightarrow \mathcal{A} / \mathcal{G}_{0} \times \Sigma$ of the bundle

$$
\mathcal{L}^{v}:=\frac{\mathcal{A} \times L_{v}}{\mathcal{G}_{0}} \rightarrow \mathcal{A} / \mathcal{G}_{0} \times \Sigma,
$$

and $\mathcal{I N D}\left(\bar{\partial}^{\nu}\right) \in K_{T}\left(\mathcal{B}_{I}\right)$ is the pullback under $\pi_{0}$ of the index bundle of the Cauchy-Riemann operators on $\mathcal{L}^{\nu}$. The torus $T$ acts trivially on $\mathcal{A} / \mathcal{G}_{0} \times \Sigma$ and by $\rho_{\nu}$ on the fibres of $\mathcal{L}^{v}$. Hence by Example 4.7,

$$
e_{T}\left(\mathcal{I N} \mathcal{D}\left(\bar{\partial}^{\nu}, \mathcal{L}^{\nu}\right)\right)=\sum_{j \geq 0}\left\langle\mathrm{w}_{v}, \xi\right\rangle^{d_{\nu}+1-g-j} c_{j}\left(\mathcal{I N} \mathcal{D}\left(\bar{\partial}^{\nu}, \mathcal{L}^{v}\right)\right) .
$$


Hence it remains to compute the ordinary Chern classes of the $K$-theory class $\mathcal{I} \mathcal{N} \mathcal{D}\left(\bar{\partial}^{v}, \mathcal{L}^{v}\right)$. The Atiyah-Singer index theorem for families asserts that

$$
\operatorname{ch}\left(\mathcal{I} \mathcal{N} \mathcal{D}\left(\bar{\partial}^{v}, \mathcal{L}^{v}\right)\right)=\int_{\Sigma} \operatorname{td}(T \Sigma) \operatorname{ch}\left(\mathcal{L}^{v}\right) \in H^{*}(\mathcal{A} / \mathcal{G})
$$

(See [1, Theorem 4.3] and [2, Theorem 5.1].) Here ch and td denote the Chern character and the Todd class, respectively. The Todd class of $T \Sigma$ is given by

$$
\operatorname{td}(T \Sigma)=1+(1-g) \sigma
$$

where $\sigma \in H^{2}(\Sigma ; \mathbb{Z})$ denotes the positive generator. Thus our task at hand is to compute the Chern character of the line bundle $\mathcal{L}^{v}$. By Lemma 4.13 below, the first Chern class of $\mathcal{L}^{v}$ is given by

$$
c_{1}\left(\mathcal{L}^{\nu}\right)=d_{\nu} \sigma-\sum_{i=1}^{2 g} \sum_{j=1}^{k} \mathrm{w}_{\nu j} \alpha_{i} \wedge \tau_{i j}
$$

From this we can compute $\operatorname{ch}\left(\mathcal{L}^{v}\right)$. Note that $\alpha_{i} \wedge \alpha_{i^{\prime}}= \pm \sigma$ whenever $i^{\prime}=i \pm g$ and is equal to zero otherwise. Hence

$$
\begin{aligned}
\frac{1}{2}\left(\sum_{i=1}^{2 g} \sum_{j=1}^{k} \mathrm{w}_{\nu j} \alpha_{i} \wedge \tau_{i j}\right)^{2} & =-\sigma \wedge \sum_{i=1}^{g} \sum_{j, j^{\prime}=1}^{k} \mathrm{w}_{\nu j} \mathrm{w}_{\nu j^{\prime}} \tau_{i j} \wedge \tau_{i+g, j^{\prime}} \\
= & :-\sigma \wedge \Omega_{\nu}
\end{aligned}
$$

and all higher powers vanish. It follows that

$$
\operatorname{ch}\left(\mathcal{L}^{\nu}\right)=1+d_{\nu} \sigma-\sum_{i=1}^{2 g} \sum_{j=1}^{k} \mathrm{w}_{\nu j} \alpha_{i} \wedge \tau_{i j}-\sigma \wedge \Omega_{\nu}
$$

Applying the index theorem for families we obtain

$$
\begin{aligned}
\operatorname{ch}\left(\mathcal{I N D}\left(\bar{\partial}^{v}, \mathcal{L}^{v}\right)\right) & =\int_{\Sigma} \operatorname{td}(T \Sigma) \operatorname{ch}\left(\mathcal{L}^{v}\right) \\
& =\int_{\Sigma}\left(\left(d_{v}+1-g\right) \sigma-\sum_{i=1}^{2 g} \sum_{j=1}^{k} \mathrm{w}_{v j} \alpha_{i} \wedge \tau_{i j}-\sigma \wedge \Omega_{v}\right) \\
& =d_{v}+1-g-\Omega_{v} .
\end{aligned}
$$

The last formula implies, by a standard algebraic argument, that

$$
c_{1}\left(\mathcal{I} \mathcal{N} \mathcal{D}\left(\bar{\partial}^{v}, \mathcal{L}^{v}\right)\right)=-\Omega_{v}, \quad c_{j}\left(\mathcal{I} \mathcal{N} \mathcal{D}\left(\bar{\partial}^{v}, \mathcal{L}^{v}\right)\right)=\frac{1}{j !} c_{1}\left(\mathcal{I} \mathcal{N} \mathcal{D}\left(\bar{\partial}^{v}, \mathcal{L}^{v}\right)\right)^{j}
$$


Hence, by (40),

$$
\begin{aligned}
e_{T}\left(\mathcal{I N D}\left(\bar{\partial}^{v}, \mathcal{L}^{\nu}\right)\right) & =\sum_{j \geq 0}\left\langle\mathrm{w}_{v}, \xi\right\rangle^{d_{\nu}+1-g-j} \frac{\left(-\Omega_{v}\right)^{j}}{j !} \\
& =\left\langle\mathrm{w}_{\nu}, \xi\right\rangle^{d_{\nu}+1-g} \exp \left(-\frac{\Omega_{v}}{\left\langle\mathrm{w}_{\nu}, \xi\right\rangle}\right) .
\end{aligned}
$$

Since $e_{T}\left(\mathcal{I} \mathcal{N} \mathcal{D}\left(\bar{\partial}^{v}\right)\right)=\pi_{0}^{*} e_{T}\left(\mathcal{I} \mathcal{N} \mathcal{D}\left(\bar{\partial}^{v}, \mathcal{L}^{v}\right)\right)$ the result follows.

It remains to prove

Lemma 4.13. The first Chern class of $\mathcal{L}^{v}$ is given by

$$
c_{1}\left(\mathcal{L}^{\nu}\right)=d_{\nu} \sigma-\sum_{i=1}^{2 g} \sum_{j=1}^{k} \mathrm{w}_{\nu j} \alpha_{i} \wedge \tau_{i j} .
$$

Proof. Fix a reference connection $A_{0} \in \mathcal{A}(P)$ and denote by $\mathcal{A}_{0} \subset \mathcal{A}(P)$ the set of connections $A \in \mathcal{A}(P)$ that satisfy $F_{A}=\operatorname{Vol}(\Sigma)^{-1} \lambda(P) \mathrm{dvol}_{\Sigma}$ and $d^{*}$ $\left(A-A_{0}\right)=0$. The restricted gauge group $\mathcal{G}_{00} \subset \mathcal{G}_{0}(P)$ consists of all gauge transformations $g: \Sigma \rightarrow T$ that satisfy $d^{*}\left(g^{-1} d g\right)=0$ and $g\left(z_{0}\right)=1$ l. Identify the quotient $\mathcal{A}_{0} / \mathcal{G}_{00}$ with the standard torus $\mathbb{T}^{2 g k}$ via the map

$$
\mathbb{R}^{2 g k} \rightarrow \mathcal{A}_{0}: t \mapsto A_{t}:=A_{0}+\sum_{i=1}^{2 g} \sum_{j=1}^{k} t_{i j} \alpha_{i} e_{j} .
$$

For $i$ and $j$ let $g_{i j} \in \mathcal{G}_{00}$ be the unique harmonic gauge transformation that satisfies $g_{i j}^{-1} d g_{i j}=\alpha_{i} e_{j}$ (and $g_{i j}\left(z_{0}\right)=11$ ). Then the restriction of $\mathcal{L}^{v}$ to the submanifold $\mathcal{A}_{0} / \mathcal{G}_{00} \times \Sigma$ can be identified with the quotient $\mathbb{R}^{2 g k} \times L_{v} / \mathbb{Z}^{2 g k}$, where $m=\left\{m_{i j}\right\} \in \mathbb{Z}^{2 g k}$ acts by

$$
m^{*}(t, z, v):=\left(t+m, z, \prod_{i j} \rho_{\nu}\left(g_{i j}(z)\right)^{-m_{i j}} v\right) .
$$

A section is a map $\mathbb{R}^{2 g k} \times \Sigma \rightarrow L_{v}:(t, z) \mapsto s(t, z)=s_{t}(z) \in L_{v z}$ that satisfies

$$
s(t+m, z)=\prod_{i j} \rho_{\nu}\left(g_{i j}(z)\right)^{-m_{i j}} s(t, z), \quad m \in \mathbb{Z}^{2 g k} .
$$

A connection is given by the formula

$$
d^{\nabla} s:=d_{A_{t}} s_{t}+\sum_{i=1}^{2 g} \sum_{j=1}^{k} \frac{\partial s_{t}}{\partial t_{i j}} d t_{i j} .
$$


An easy computation shows that the curvature of this connection is the 2-form $F^{\nabla} \in \Omega^{2}\left(\mathbb{T}^{2 g k} \times \Sigma, \sqrt{-1} \mathbb{R}\right)$ given by

$$
\begin{aligned}
F^{\nabla} & =\dot{\rho}_{\nu}\left(F_{A_{t}}\right)-\sum_{i=1}^{2 g} \sum_{j=1}^{k} \dot{\rho}_{\nu}\left(\frac{\partial A_{t}}{\partial t_{i j}}\right) \wedge d t_{i j} \\
& =-2 \pi \sqrt{-1}\left\langle\mathrm{w}_{\nu}, \lambda(P)\right\rangle \frac{\mathrm{dvol}_{\Sigma}}{\operatorname{Vol}(\Sigma)}+\sum_{i=1}^{2 g} \sum_{j=1}^{k} 2 \pi \sqrt{-1}\left\langle\mathrm{w}_{\nu}, e_{j}\right\rangle \alpha_{i} \wedge d t_{i j}
\end{aligned}
$$

Since the first Chern class of $\mathcal{L}^{v}$ is represented by the 2 -form $\sqrt{-1} F^{\nabla} / 2 \pi$, the result follows.

\section{Computation of the genus zero invariants}

Let $\mathcal{I}$ denote the set of partitions

$$
I_{1} \cup \ldots \cup I_{k}=\{1, \ldots, n\}
$$

that satisfy the (Dimension) condition in the introduction, i.e. for every $j \in$ $\{1, \ldots, k\}$, the subspace

$$
E_{j}:=\operatorname{span}\left\{\mathrm{w}_{v} \mid v \in I_{1} \cup \cdots \cup I_{j}\right\} \subset \mathfrak{t}^{*}
$$

has dimension $j$ and $\mathrm{w}_{v} \notin E_{j}$ for every $v \in I_{j+1} \cup \cdots \cup I_{k}$. It is now convenient to fix an orientation of $\mathrm{t}$. For every $I=\left(I_{1}, \ldots, I_{k}\right) \in \mathcal{I}$ we introduce the iterated residue $\phi_{\lambda}^{I}=\phi_{\lambda}^{\rho, I}: S^{*}\left(\mathfrak{t}^{*}\right) \rightarrow \mathbb{R}$ by

$$
\phi_{\lambda}^{I}(\alpha):=\frac{1}{(2 \pi i)^{k}} \oint \cdots \oint \frac{\alpha\left(\sum z_{j} e_{j}\right)}{\prod_{\nu=1}^{n}\left\langle\mathrm{w}_{\nu}, \sum z_{j} e_{j}\right\rangle^{d_{v}+1}} d z_{k} \cdots d z_{1},
$$

where $d_{v}:=\left\langle\mathrm{w}_{v}, \lambda\right\rangle$. Here the lattice vectors $e_{1}, \ldots, e_{k} \in \mathfrak{t}$ form an oriented integral basis of $\Lambda$ such that the vectors $e_{j}, \ldots, e_{k}$ are orthogonal to the span of the vectors $\mathrm{w}_{v}$ for $v \in I_{1} \cup \cdots \cup I_{j-1}$ and $2 \leq j \leq k$. These requirements determine the $e_{j}$ up to a change $e_{j}^{\prime}= \pm e_{j}+\sum_{i>j} a_{i j} e_{i}$. The corresponding coordinates $\xi=\sum z_{j} e_{j}=\sum z_{j}^{\prime} e_{j}^{\prime}$ change by $z_{i}= \pm z_{i}^{\prime}+\sum_{j<i} a_{i j} z_{j}^{\prime}$. Since the $e_{i}$ and the $e_{i}^{\prime}$ form oriented bases there is an even number of minus signs. It follows from these observations and Remark 3.2 that the integral (41) is independent of the choice of the $e_{i}$.

Lemma 5.1. For every regular value $\tau$ of $\mu$ there exists a collection of integers $\left\{m_{I}\right\}_{I \in \mathcal{I}}$ such that

$$
\Phi_{\lambda}^{\rho, \tau}(\alpha)=\sum_{I \in \mathcal{I}} m_{I} \phi_{\lambda}^{I}(\alpha)
$$


for every $\lambda \in \Lambda$ and every $\alpha \in S^{*}\left(\mathfrak{t}^{*}\right)$. Moreover, given a path $\gamma$ connecting $\tau$ to $\mathfrak{t}^{*} \backslash \operatorname{im} \mu$, the coefficients $m_{I}$ can be chosen to satisfy the following condition: If $I=\left(I_{1}, \ldots, I_{k}\right) \in \mathcal{I}$ is such that $\gamma$ does not intersect the hyperplane spanned by the $\mathrm{w}_{v}$ for $v \in I_{1} \cup \cdots \cup I_{k-1}$, then $m_{I}=0$.

Proof. Theorem 1.1 and induction over the dimension of $T$.

Remark 5.2. Fix an oriented basis $e_{1}, \ldots, e_{k}$ of $\mathfrak{t}$, let $H_{v} \subset \mathbb{C}^{k}$ be the hyperplane $\sum_{j=1}^{k}\left\langle\mathrm{w}_{\nu}, e_{j}\right\rangle z_{\nu}=0$, and denote $H:=\bigcup_{\nu=1}^{n} H_{\nu}$. Then the right hand side of (41) can be interpreted as the integral of the $k$-form

$$
\omega_{\lambda, \alpha}:=\frac{\alpha\left(\sum z_{j} e_{j}\right)}{(2 \pi i)^{k} \prod_{\nu=1}^{n}\left\langle\mathrm{w}_{\nu}, \sum z_{j} e_{j}\right\rangle^{\left\langle\mathrm{w}_{v}, \lambda\right\rangle+1}} d z_{1} \wedge \cdots \wedge d z_{k} \in \Omega^{k}\left(\mathbb{C}^{k} \backslash H\right)
$$

over a suitable homology class $\sigma_{I} \in H_{k}\left(\mathbb{C}^{k} \backslash H\right)$. Hence, by Lemma 5.1, there is a locally constant map $\tau \mapsto \sigma(\tau):=\sum_{I \in \mathcal{I}} m_{I}(\tau) \sigma_{I}$ which assigns to every regular value of $\mu$ a homology class $\sigma(\tau) \in H_{k}\left(\mathbb{C}^{k} \backslash H\right)$ such that the invariant $\Phi_{\lambda}^{\rho, \tau}(\alpha)$ is equal to the integral of $\omega_{\lambda, \alpha}$ over $\sigma(\tau)$ (for all $\lambda$ and $\alpha$ ). It is an interesting problem to study the map $\tau \mapsto \sigma(\tau)$ in more detail.

Lemma 5.3. For $v=1, \ldots, n$ let $\ell_{v}$ be integers and $a_{v}, b_{v}$ be real numbers such that $a_{v} \neq 0$. If $\sum_{v=1}^{n} \ell_{v}=-1$ then

$$
\frac{1}{2 \pi i} \oint \prod_{\nu=1}^{n}\left(a_{v} z+b_{v}\right)^{\ell_{v}} d z=\prod_{\nu=1}^{n} a_{v}^{\ell_{\nu}} .
$$

If $\sum_{v=1}^{n} \ell_{v}<-1$ then the integral is zero.

Proof. In the variable $w:=1 / z$ the integrand reads

$$
\prod_{\nu}\left(\frac{a_{v}}{w}+b_{v}\right)^{\ell_{v}}\left(\frac{-d w}{w^{2}}\right)=-\frac{\prod_{\nu}\left(a_{v}+b_{v} w\right)^{\ell_{\nu}} d w}{w^{2+\sum \ell_{v}}} .
$$

Since $a_{v} \neq 0$ the numerator is holomorphic near the origin. Hence the residue is zero whenever $2+\sum_{v} \ell_{v} \leq 0$ and is equal to $\prod_{\nu} a_{v}^{\ell_{v}}$ whenever $2+\sum_{\nu} \ell_{v}=1$.

Lemma 5.4. Let $I \in \mathcal{I}$ and $e_{1}, \ldots, e_{k}$ be a positive basis of $\Lambda$ such that $e_{j}, \ldots, e_{k}$ are orthogonal to $\mathrm{w}_{v}$ for $v \in I_{1} \cup \cdots \cup I_{j-1}$ (as in (41)). Let $\lambda \in \Lambda$ and $\ell$ be an n-tuple of nonnegative integers such that

$$
|\ell|=n-k+\sum_{\nu=1}^{n} d_{\nu}, \quad d_{\nu}:=\left\langle\mathrm{w}_{\nu}, \lambda\right\rangle .
$$

If $I \in \mathcal{I}_{\lambda}(\ell)$ then

$$
\phi_{\lambda}^{I}\left(\mathrm{w}^{\ell}\right)=\prod_{j=1}^{k} \prod_{v \in I_{j}}\left\langle\mathrm{w}_{v}, e_{j}\right\rangle^{\ell_{v}-d_{v}-1} .
$$

Otherwise $\phi_{\lambda}^{I}\left(\mathrm{w}^{\ell}\right)=0$. 
Proof. The condition $I \in \mathcal{I}_{\lambda}(\ell)$ asserts that

$$
\sum_{v \in I_{j}}\left(\ell_{v}-d_{v}-1\right)=-1
$$

for $j=1, \ldots, k$. Consider the integral over $z_{k}$. The coefficient $\left\langle\mathrm{w}_{v}, e_{k}\right\rangle$ of $z_{k}$ in the linear map $\left(z_{1}, \ldots, z_{k}\right) \mapsto\left\langle\mathrm{w}_{v}, \sum_{j} z_{j} e_{j}\right\rangle$ is nonzero iff $v \in I_{k}$. So, by Lemma 5.3,

$$
\begin{aligned}
\phi_{\lambda}^{I}\left(\mathrm{w}^{\ell}\right)= & \left(\prod_{v \in I_{k}}\left\langle\mathrm{w}_{\nu}, e_{k}\right\rangle^{\ell_{\nu}-d_{\nu}-1}\right) \frac{1}{(2 \pi i)^{k-1}} \\
& \oint \cdots \oint \prod_{j=1}^{k-1} \prod_{\nu \in I_{j}}\left\langle\mathrm{w}_{\nu}, \sum_{j=1}^{k-1} z_{j} e_{j}\right\rangle^{\ell_{\nu}-d_{\nu}-1} d z_{k-1} \ldots d z_{1}
\end{aligned}
$$

whenever $\sum_{v \in I_{k}}\left(\ell_{v}-d_{v}-1\right)=-1$. If $\sum_{v \in I_{k}}\left(\ell_{v}-d_{v}-1\right)<-1$ then the integral over $z_{k}$ is zero. Hence it follows by induction that $\phi_{\lambda}^{I}\left(\mathrm{w}^{\ell}\right)$ has the required form whenever $I \in \mathcal{I}_{\lambda}(\ell)$. If $I \notin \mathcal{I}_{\lambda}(\ell)$ then it follows from (42) that $\sum_{v \in I_{j}}\left(\ell_{v}-d_{v}\right.$ $-1)<-1$ for some $j$ and hence $\phi_{\lambda}^{I}\left(\mathrm{w}^{\ell}\right)=0$.

Proof of Theorem 1.2. Assertion (i) follows from Lemmata 5.1 and 5.4.

We prove (ii). Let $\lambda \in \Lambda, d_{v}:=\left\langle\mathrm{w}_{v}, \lambda\right\rangle, \ell$ be an $n$-tuple of nonnegative integers, $J \subset\{1, \ldots, n\}$ be an index set such that $\left\{\mathrm{w}_{v} \mid v \in J\right\}$ is a basis of $\mathrm{t}^{*}$, and assume that $\ell_{v}=d_{v}$ for $v \in J$ and $\ell_{v}=d_{v+1}$ for $v \notin J$. Then a partition $I$ belongs to $\mathcal{I}_{\lambda}(\ell)$ if and only if $I \in \mathcal{I}$ and $I_{j} \cap J$ consists of a single element for each $j$. This follows from the equation $\sum_{v \in I_{j}}\left(\ell_{v}-d_{v}-1\right)=-1$ and the fact that each summand $\ell_{v}-d_{v}-1$ equals 0 or -1 .

Assume

$$
\tau \notin C(J)=\left\{\sum_{v \in J} \eta_{v} \mathrm{w}_{v} \mid \eta_{v} \geq 0\right\} .
$$

We must prove that $\Phi_{\lambda}^{\rho, \tau}\left(\mathrm{w}^{\ell}\right)=0$. To see this, we examine the set $\mathcal{I}_{\lambda}(\ell)$. Since the set $\left\{\mathrm{w}_{v} \mid v \in J\right\}$ is linearly independent it follows that, for each ordering $J=\left\{v_{1}, \ldots, v_{k}\right\}$, there exists a unique partition $I \in \mathcal{I}_{\lambda}(\ell)$ such that $v_{j} \in I_{j}$ for all $j$, and conversely, each partition $I \in \mathcal{I}_{\lambda}(\ell)$ determines an ordering of $J$. Moreover, for every such partition the hyperplane

$$
W_{I}:=\operatorname{span}\left\{\mathrm{w}_{v} \mid v \notin I_{k}\right\}
$$

agrees with the hyperplane

$$
W_{\nu_{k}}:=\operatorname{span}\left\{\mathrm{w}_{\nu} \mid v \in J \backslash\left\{v_{k}\right\}\right\} .
$$

Hence the hyperplanes $\mathrm{W}_{I}$ for $I \in \mathcal{I}_{\lambda}(\ell)$ are precisely the supporting hyperplanes of $C(J)$. Since $\tau \notin C(J)$, there exists a straight line $\gamma$ connecting $\tau$ to $t^{*} \backslash \operatorname{im} \mu$ 
which misses the supporting hyperplanes (this is true because $C(J)$ is a cone over a simplex). Hence the coefficients $m_{I}$ in Lemma 5.1 can be chosen such that $m_{I}=0$ for every $I \in \mathcal{I}_{\lambda}(\ell)$. This implies that

$$
\Phi_{\lambda}^{\rho, \tau}\left(\mathrm{w}^{\ell}\right)=\sum_{I \in \mathcal{I}_{\lambda}(\ell)} m_{I} \phi_{\lambda}^{I}\left(\mathrm{w}^{\ell}\right)=0 .
$$

The same argument shows that the invariant $\Phi_{\lambda}^{\rho, \tau}\left(\mathrm{w}^{\ell}\right)$ for $\tau \in C(J)$ is independent of $\tau$.

Assume $\tau \in C(J)$. Fix an ordering $J=\left\{v_{1}, \ldots, v_{k}\right\}$ and let $I \in \mathcal{I}_{\lambda}(\ell)$ be the unique partition satisfying $v_{j} \in I_{j}$ for $j=1, \ldots, k$. Choose an integer basis $e_{1}, \ldots, e_{k}$ of $\mathfrak{t}$ such that $\left\langle\mathrm{w}_{v}, e_{j}\right\rangle=0$ for $v \in I_{1} \cup \cdots I_{j-1}$ and $\left\langle\mathrm{w}_{v_{j}}, e_{j}\right\rangle>0$. Let $\tau_{0}$ be a positive linear combination of $\mathrm{w}_{v_{1}}, \ldots, \mathrm{w}_{v_{k-1}}$. Since the invariant is independent of the choice of $\tau \in C(J)$, we may assume $\tau=\tau_{0}+\varepsilon \tau_{1}$, where $\tau_{1}:=\mathrm{w}_{v_{k}}$. Since the invariant is zero outside of $C(J)$, we have

$$
\Phi_{\lambda}^{\rho, \tau_{0}-\varepsilon \tau_{1}}\left(\mathrm{w}^{\ell}\right)=0 .
$$

Hence, by Theorem 1.1,

$$
\Phi_{\lambda}^{\rho, \tau}\left(\mathrm{w}^{\ell}\right)=\frac{1}{\left\langle\mathrm{w}_{v_{k}}, e_{k}\right\rangle} \Phi_{\lambda_{0}}^{\rho_{0}, \tau_{0}}\left(\prod_{j=1}^{k-1} \prod_{v \in I_{j}} \mathrm{w}_{v}^{\ell_{v}}\right) .
$$

Now assertion (ii) follows by induction.

We prove (iii). Assume $|\ell|=n-k+\sum_{v=1}^{n} d_{v}$ (otherwise both invariants are zero). Since

$$
\ell_{v}-d_{v}-1=\ell_{v}+d_{v}^{\prime}-\left(d_{v}+d_{v}^{\prime}\right)-1,
$$

for every $v$ we have

$$
\mathcal{I}_{\lambda}(\ell)=\mathcal{I}_{\lambda+\lambda^{\prime}}\left(\ell+d^{\prime}\right), \quad \phi_{\lambda}^{I}\left(\mathrm{w}^{\ell}\right)=\phi_{\lambda+\lambda^{\prime}}^{I}\left(\mathrm{w}^{\ell+d^{\prime}}\right)
$$

for every $I \in \mathcal{I}$ (see Lemma 5.4). Hence (iii) follows from Lemma 5.1.

To prove (iv) and (v) we introduce the following notation. For every $\lambda \in \Lambda$ and every $n$-tuple $\ell$ of nonnegative integers define the number

$$
\iota_{\lambda}(\ell):=\sum_{d_{\nu} \geq 0} \max \left\{\ell_{\nu}-d_{\nu}-1,0\right\}+\sum_{d_{\nu} \leq-1} \ell_{\nu}, \quad d_{\nu}:=\left\langle\mathrm{w}_{\nu}, \lambda\right\rangle .
$$

Recall that $J_{\ell}:=\left\{v \mid \ell_{v} \leq d_{v}\right\}$. We prove (iv) and (v) in five steps.

Step 1. Each $\mathrm{w}^{\ell}$ can be expressed as a linear combination of classes $\mathrm{w}^{\ell^{\prime}}$ that satisfy $J_{\ell^{\prime}} \subset J_{\ell}$ and either $\mathcal{I}_{\lambda}\left(\ell^{\prime}\right)=\emptyset$ or $\ell_{\nu}^{\prime} \leq \max \left\{d_{v}+1,0\right\}$ for all $v$. 
We prove Step 1 by induction over $\iota_{\lambda}(\ell)$. If $\iota_{\lambda}(\ell)=0$ then $\ell_{v}^{\prime} \leq \max \left\{d_{v}+1,0\right\}$ for all $v$. Assume $\iota_{\lambda}(\ell)>0$ and, by induction, that the claim has been established for every $\ell^{\prime}$ that satisfies $\iota_{\lambda}\left(\ell^{\prime}\right)<\iota_{\lambda}(\ell)$. If $\mathcal{I}_{\lambda}(\ell)=\emptyset$ there is nothing to prove. Hence assume $\mathcal{I}_{\lambda}(\ell) \neq \emptyset$. Since $\iota_{\lambda}(\ell)>0$ there is a $\nu_{0}$ such that $\ell_{\nu_{0}}>0$ and $\ell_{v_{0}}>d_{v_{0}}+1$. Let $I=\left(I_{1}, \ldots, I_{k}\right) \in \mathcal{I}_{\lambda}(\ell)$. Since $\sum_{v \in I_{j}}\left(\ell_{v}-d_{v}-1\right)=-1$ for every $j$ there are indices $v_{j} \in I_{j}$ for $j=1, \ldots, k$ such that $\ell_{v_{j}} \leq d_{v_{j}}$. By the (Dimension) condition, the vectors $\mathrm{w}_{v_{1}}, \ldots, \mathrm{w}_{v_{k}}$ form a basis of $\mathfrak{t}^{*}$. Hence $\mathrm{w}_{v_{0}}$ can be expressed as a linear combination of the vectors $\mathrm{w}_{v_{j}}$. Since $\ell_{v_{0}}>0$ we can replace one of the factors $\mathrm{w}_{\nu_{0}}$ in $\mathrm{w}^{\ell}$ by this linear combination. This expresses $\mathrm{w}^{\ell}$ as a linear combination of monomials of the form $\mathrm{w}^{\ell^{\prime}}$ with $\iota_{\lambda}\left(\ell^{\prime}\right)<\iota_{\lambda}(\ell)$ and $J_{\ell^{\prime}} \subset J_{\ell}$. Hence the assertion for $\mathrm{w}^{\ell}$ follows from the induction hypothesis.

Step 2. If $\ell_{v} \leq d_{v}+1$ for every $v$ and $\mathcal{I}_{\lambda}(\ell) \neq \emptyset$ then $\ell$ satisfies (ii).

Let $I \in \mathcal{I}_{\lambda}(\ell)$. Then the formula $\sum_{v \in I_{j}}\left(\ell_{v}-d_{v}-1\right)=-1$ shows that, for each $j$, there is precisely one index $v_{j} \in I_{j}$ such that $\ell_{v_{j}}=d_{v_{j}}$ and $\ell_{v}=d_{v}+1$ for $v \in I_{j} \backslash\left\{v_{j}\right\}$. Since the vectors $\mathrm{w}_{v_{1}}, \ldots, \mathrm{w}_{v_{k}}$ form a basis of $\mathfrak{t}^{*}$, it follows that $\ell$ satisfies (ii) with $J=\left\{v_{1}, \ldots, v_{k}\right\}$.

Step 3. We prove (iv).

Assume $d_{v} \geq-1$ for every $v$. Then, by Step 1 , each $\mathrm{w}^{\ell} \in S^{m_{\lambda}}\left(\mathfrak{t}^{*}\right)$ is a linear combination of classes $\mathrm{w}^{\ell^{\prime}}$ that satisfy either $\mathcal{I}_{\lambda}\left(\ell^{\prime}\right)=\emptyset$ or $\ell_{v}^{\prime} \leq d_{v}+1$ for all $v$. Hence the assertion follows from Step 2.

Step 4. (v) holds under the assumption $d_{v} \geq-1$ for all $v$.

We argue indirectly and assume that $\Phi_{\lambda}^{\rho, \tau}\left(\mathrm{w}^{\ell}\right) \neq 0$. Then the linear combination in Step 1 must contain a term $\mathrm{w}^{\ell^{\prime}}$ that satisfies $J_{\ell^{\prime}} \subset J_{\ell}$, and $\Phi_{\lambda}^{\rho, \tau}\left(\mathrm{w}^{\ell^{\prime}}\right) \neq 0$. The latter implies that $\mathcal{I}_{\lambda}\left(\ell^{\prime}\right) \neq \emptyset$ and so $\ell_{v}^{\prime} \leq d_{v}+1$ for all $v$. Hence, by Step 2, $\ell^{\prime}$ satisfies (ii) with $J=J_{\ell^{\prime}}=\left\{v \mid \ell_{v}^{\prime} \leq d_{v}\right\}$. Since $\Phi_{\lambda}^{\rho, \tau}\left(\mathrm{w}^{\ell^{\prime}}\right) \neq 0$, it follows from (ii) that $\tau \in C\left(J_{\ell^{\prime}}\right) \subset C\left(J_{\ell}\right)$.

Step 5. We prove (v).

Suppose $\Phi_{\lambda}\left(\mathrm{w}^{\ell}\right) \neq 0$. Choose $\lambda^{\prime}$ such that

$$
d_{\nu}^{\prime}:=\left\langle\mathrm{w}_{\nu}, \lambda^{\prime}\right\rangle \geq \max \left\{0,-1-d_{v}\right\}
$$

for all $v$. Then, by (iii), we have

$$
\Phi_{\lambda+\lambda^{\prime}}\left(\mathrm{w}^{\ell+d^{\prime}}\right)=\Phi_{\lambda}\left(\mathrm{w}^{\ell}\right) \neq 0 .
$$

Hence, by Step 4, $\tau \in C(J)$, where

$$
J:=\left\{v \mid \ell_{v}+d_{v}^{\prime} \leq\left\langle\mathrm{w}_{v}, \lambda+\lambda^{\prime}\right\rangle\right\}=\left\{v \mid \ell_{v} \leq d_{v}\right\} .
$$

This proves the theorem. 


\section{Quantum cohomology}

Let $\tau$ be a regular value of $\mu$. Throughout this section we assume that $T$ acts freely on $\mu^{-1}(\tau)$. Equivalently, if $J \subset\{1, \ldots, n\}$ is an index set consisting of $k$ elements such that $\tau \in C(J)$, then $\tau$ belongs to the interior of $C(J)$ and the determinant of the tuple $\left\{\mathrm{w}_{v} \mid v \in J\right\}$ is equal to plus or minus one. Under this assumption the symplectic quotient

$$
\bar{M}:=M / / T(\tau)=\mu^{-1}(\tau) / T
$$

is a Kähler manifold. We denote by $H^{*}(\bar{M})$, respectively $H_{*}(\bar{M})$, the quotients of the integral (co)homology groups by their torsion subgroups. By Kirwan's theorem, the homomorphism $H^{*}(B T) \rightarrow H^{*}(\bar{M})$ is surjective and the homomorphism $H_{*}(\bar{M}) \rightarrow H_{*}(B T)$ is injective. For every $v$ denote by $\overline{\mathrm{w}}_{v} \in H^{2}(\bar{M})$ the image of the cohomology class $\mathrm{w}_{v} \in \Lambda^{*} \cong H^{2}(B T)$ under the Kirwan homomorphism $H^{2}(B T) \rightarrow H^{2}(\bar{M})$. By Theorem E.4, the cohomology class $\overline{\mathrm{w}}_{v}$ vanishes whenever $\tau \notin C(\{1, \ldots, n\} \backslash\{v\})$. The remaining classes $\overline{\mathrm{w}}_{v}$ generate $H^{2}(\bar{M})$. Hence the image of the homomorphism $H_{2}(\bar{M}) \rightarrow H_{2}(B T) \cong \Lambda$ is the subgroup

$$
\Lambda(\tau):=\left\{\lambda \in \Lambda \mid \tau \notin C(\{1, \ldots, n\} \backslash\{\nu\}) \Longrightarrow\left\langle\mathrm{w}_{\nu}, \lambda\right\rangle=0\right\} .
$$

Recall the definition of the inverse isomorphism $\Lambda(\tau) \rightarrow H_{2}(\bar{M}): \lambda \mapsto \bar{\lambda}$ and the effective cone $\Lambda_{\text {eff }}(\tau) \subset \Lambda(\tau)$

$$
\Lambda_{\text {eff }}(\tau):=\left\{\lambda \in \Lambda(\tau) \mid\left\langle\tau^{\prime}, \lambda\right\rangle \geq 0 \text { for all } \tau^{\prime} \in C(\tau)\right\},
$$

where $C(\tau)$ denotes the chamber of $\tau$. Note that

$$
\left\langle\tau^{\prime}, \lambda\right\rangle>0 \quad \text { for } \lambda \in \Lambda_{\text {eff }}(\tau) \backslash\{0\}, \tau^{\prime} \in C(\tau) .
$$

Denote by $\mathcal{D}_{\text {eff }}(\tau) \subset \mathbb{Z}^{n}$ the cone

$$
\mathcal{D}_{\text {eff }}(\tau):=\left\{\left(\left\langle\mathrm{w}_{1}, \lambda\right\rangle, \ldots,\left\langle\mathrm{w}_{n}, \lambda\right\rangle\right) \mid \lambda \in \Lambda_{\text {eff }}(\tau)\right\} .
$$

Note that the map $\Lambda_{\text {eff }}(\tau) \rightarrow \mathcal{D}_{\text {eff }}(\tau)$ is a bijection. We denote the inverse by $\mathcal{D}_{\text {eff }}(\tau) \rightarrow \Lambda_{\text {eff }}(\tau): d \mapsto \lambda_{d}$. We emphasize that $\mathcal{D}_{\text {eff }}(\tau)$ is not necessarily contained in the positive quadrant of $\mathbb{Z}^{n}$.

Let us now consider the vector

$$
\tau:=\sum_{\nu=1}^{n} \mathrm{w}_{\nu} .
$$

(We still assume that $T$ acts freely on $\mu^{-1}(\tau)$.) Then $\bar{M}$ is a monotone symplectic manifold (see Lemma E.3). The genus zero Gromov-Witten invariants of $\bar{M}$ with fixed marked points in a homology class $\bar{\lambda} \in H_{2}(\bar{M})$ are denoted by

$$
\mathrm{GW}_{\bar{\lambda}}^{\bar{M}}: H^{*}(\bar{M}) \times \cdots \times H^{*}(\bar{M}) \rightarrow \mathbb{Z} .
$$


The number of arguments will in each case be clear from the context. For an $n$-tuple $\ell=\left(\ell_{1}, \ldots, \ell_{n}\right)$ of nonnegative integers and a cohomology class $\bar{\alpha} \in H^{*}(\bar{M})$ we abbreviate

$$
\operatorname{GW}_{\bar{\lambda}}^{\bar{M}}\left(\overline{\mathrm{w}}^{* \ell}, \bar{\alpha}\right):=\operatorname{GW}_{\bar{\lambda}}^{\bar{M}}\left(\overline{\mathrm{w}}_{1}, \ldots, \overline{\mathrm{w}}_{1}, \ldots, \overline{\mathrm{w}}_{n}, \ldots, \overline{\mathrm{w}}_{n}, \bar{\alpha}\right),
$$

where each argument $\overline{\mathrm{w}}_{v}$ occurs $\ell_{v}$ times. Since the Gromov-Witten invariants are invariant under symplectic deformation, we have

$$
\lambda \in \Lambda(\tau) \backslash \Lambda_{\text {eff }}(\tau) \quad \Longrightarrow \quad \mathrm{GW}_{\bar{\lambda}}^{\bar{M}} \equiv 0 .
$$

Lemma 6.1. Let $\tau:=\sum_{v=1}^{n} \mathrm{w}_{v}$, suppose that $T$ acts freely on $\mu^{-1}(\tau)$, and assume that the minimal Chern number $N$ of $\bar{M}$ is greater that one. Then for every $d$ $\in \mathcal{D}_{\text {eff }}(\tau)$, every $\lambda \in \Lambda(\tau)$, and every $\bar{\alpha} \in H^{*}(\bar{M})$, we have

$$
\mathrm{GW}_{\bar{\lambda}}^{\bar{M}}\left(\overline{\mathrm{w}}^{* d^{+}}, \bar{\alpha}\right)=\mathrm{GW}_{\bar{\lambda}-\bar{\lambda}_{d}}^{\bar{M}}\left(\overline{\mathrm{w}}^{* d^{-}}, \bar{\alpha}\right),
$$

where the $n$-tuples $d^{+}$and $d^{-}$are defined by

$$
d_{v}^{+}:=\left\{\begin{array}{r}
d_{v}, \text { if } d_{v}>0, \\
0, \text { if } d_{v} \leq 0,
\end{array} \quad d_{v}^{-}:=\left\{\begin{array}{r}
-d_{v}, \text { if } d_{v}<0, \\
0, \text { if } d_{v} \geq 0 .
\end{array}\right.\right.
$$

Proof. Let $\bar{\alpha}:=\overline{\mathrm{w}}^{\ell}$ for an $n$-tuple $\ell$ of nonnegative integers satisfying

$$
|\ell|=n-k+\langle\tau, \lambda\rangle-\left|d^{+}\right| .
$$

By Theorem 1.2 (iii), with $\lambda$ replaced by $\lambda-\lambda_{d}, \lambda^{\prime}=\lambda_{d}$, and $\ell$ replaced by $\ell+d^{-}$, we have

$$
\Phi_{\lambda}^{\rho, \tau}\left(\mathrm{w}^{d^{+}+\ell}\right)=\Phi_{\lambda-\lambda_{d}}^{\rho, \tau}\left(\mathrm{w}^{d^{-}+\ell}\right) .
$$

Hence it follows from [10, Theorem A] and the fact that $N>1$ (see equation (3) in the introduction) that

$$
\mathrm{GW}_{\bar{\lambda}}^{\bar{M}}\left(\overline{\mathrm{w}}^{*\left(d^{+}+\ell\right)}\right)=\mathrm{GW}_{\bar{\lambda}-\bar{\lambda}_{d}}^{\bar{M}}\left(\overline{\mathrm{w}}^{*\left(d^{-}+\ell\right)}\right) .
$$

Now the gluing theorem for the Gromov-Witten invariants with fixed marked points (see [13]) asserts that

$$
\begin{aligned}
\mathrm{GW}_{\bar{\lambda}}^{\bar{M}}\left(\overline{\mathrm{w}}^{* d^{+}}, \overline{\mathrm{w}}^{\ell}\right)= & \mathrm{GW}_{\bar{\lambda}}^{\bar{M}}\left(\overline{\mathrm{w}}^{*\left(d^{+}+\ell\right)}\right) \\
& -\sum_{i} \sum_{\lambda^{\prime} \neq 0} \mathrm{GW}_{\bar{\lambda}-\bar{\lambda}^{\prime}}^{\bar{M}}\left(\overline{\mathrm{w}}^{* d^{+}}, \bar{e}_{i}\right) \mathrm{GW}_{\bar{\lambda}^{\prime}}^{\bar{M}}\left(\bar{e}_{i}^{*}, \overline{\mathrm{w}}^{* \ell}\right),
\end{aligned}
$$

where the second sum is over all lattice vectors $\lambda^{\prime} \in \Lambda_{\text {eff }}(\tau) \backslash\{0\}$. Hence, by (46),

$$
\begin{aligned}
& \mathrm{GW}_{\bar{\lambda}}^{\bar{M}}\left(\overline{\mathrm{w}}^{* d^{+}}, \overline{\mathrm{w}}^{\ell}\right)-\mathrm{GW}_{\bar{\lambda}-\bar{\lambda}_{d}}^{\bar{M}}\left(\overline{\mathrm{w}}^{* d^{-}}, \overline{\mathrm{w}}^{\ell}\right) \\
& \quad=\sum_{i} \sum_{\lambda^{\prime} \neq 0} \mathrm{GW}_{\bar{\lambda}^{\prime}}^{\bar{M}}\left(\bar{e}_{i}^{*}, \overline{\mathrm{w}}^{* \ell}\right)\left(\mathrm{GW}_{\bar{\lambda}-\bar{\lambda}^{\prime}-\bar{\lambda}_{d}}^{\overline{\bar{m}}}\left(\overline{\mathrm{w}}^{* d^{-}}, \bar{e}_{i}\right)-\mathrm{GW}_{\bar{\lambda}-\bar{\lambda}^{\prime}}^{\bar{M}}\left(\overline{\mathrm{w}}^{* d^{+}}, \bar{e}_{i}\right)\right) .
\end{aligned}
$$


Note that in each summand on the right we have

$$
\begin{aligned}
\frac{1}{2} \operatorname{deg}\left(e_{i}\right) & =n-k+\left\langle\tau, \lambda-\lambda^{\prime}\right\rangle-\left|d^{+}\right| \\
& <n-k+\langle\tau, \lambda\rangle-\left|d^{+}\right| \\
& =|\ell| .
\end{aligned}
$$

Hence the assertion follows from (47) by induction over $|\ell|$.

Remark. Let $\tau, \bar{M}$ be as in Lemma 6.1 and $\lambda \in \Lambda_{\text {eff }}(\tau)$ such that $\left\langle\mathrm{w}_{\nu}, \lambda\right\rangle \geq 0$ for every $v$. Then it follows from Lemma 6.1 with $d_{v}:=\left\langle\mathrm{w}_{v}, \lambda\right\rangle$ and $\bar{\alpha}:=\mathrm{PD}(\mathrm{pt})$ that $\mathrm{GW}_{\bar{\lambda}}^{\bar{M}} \neq 0$. Hence the homology class $\bar{\lambda} \in H_{2}(\bar{M})$ can be represented by a holomorphic stable map of genus zero.

As in the introduction, let $\mathcal{R}$ be any graded commutative algebra (over the reals) with unit which is equipped with a homomorphism

$$
\Lambda_{\text {eff }}(\tau) \rightarrow \mathcal{R}: \lambda \mapsto q^{\lambda}
$$

from the additive semigroup $\Lambda_{\text {eff }}(\tau)$ to the multiplicative semigroup $\mathcal{R}$ such that

$$
\operatorname{deg}\left(q^{\lambda}\right)=2\langle\tau, \lambda\rangle .
$$

The most important example is the ring

$$
\mathcal{R}=\mathbb{R}\left[q_{1}, \ldots, q_{k}, q_{1}^{-1}, \ldots, q_{k}^{-1}\right]
$$

of polynomials with real coefficients in the variables $q_{j}$ and $q_{j}^{-1}$. To obtain the homomorphism choose a basis $e_{1}, \ldots, e_{k}$ of $\Lambda$, define the grading by $\operatorname{deg}\left(q_{j}\right)$ $=\sum_{v=1}^{n}\left\langle\mathrm{w}_{\nu}, e_{j}\right\rangle$, and the map $\lambda \mapsto q^{\lambda}$ by

$$
q^{\lambda}:=\prod_{j=1}^{k} q_{j}^{\lambda_{j}}, \quad \lambda=\sum_{j=1}^{k} \lambda_{j} e_{j} .
$$

With a more careful choice of the basis one can take $\mathcal{R}=\mathbb{R}\left[q_{1}, \ldots, q_{k}\right]$. Other possibilities are the polynomial ring $\mathcal{R}=\mathbb{R}[q]$ in one variable, the ring of polynomials in $q$ and $q^{-1}$, or the ring of Laurent series in $q$. In these cases one can choose $q$ to have degree two and define $q^{\lambda}:=q^{\langle\tau, \lambda\rangle}$. The simplest example is $\mathcal{R}=\mathbb{R}$ with the constant map $\lambda \mapsto q^{\lambda}:=1$, but then the grading has to be reduced modulo $2 N$, where $N$ is the minimal Chern number.

Given a graded algebra $\mathcal{R}$ as above define the quantum cohomology ring $\mathrm{QH}^{*}(\bar{M} ; \mathcal{R})$ as the tensor product

$$
\mathrm{QH}^{*}(\bar{M} ; \mathcal{R}):=H^{*}(\bar{M} ; \mathbb{R}) \otimes \mathcal{R}
$$


(of vector spaces over the reals). Thus an element of $\mathrm{QH}^{m}(\bar{M} ; \mathcal{R})$ is a finite sum $\bar{\alpha}=\sum_{r \in \mathcal{R}} \bar{\alpha}_{r} r$ such that $\operatorname{deg}\left(\bar{\alpha}_{r}\right)+\operatorname{deg}(r)=m$ for all $r$. The ring structure is defined by

$$
\bar{\alpha}^{\prime} * \bar{\alpha}^{\prime \prime}:=\sum_{i} \sum_{\lambda \in \Lambda_{\mathrm{eff}}(\tau)} \sum_{r^{\prime}, r^{\prime \prime}} \mathrm{GW}_{\bar{\lambda}}^{\bar{M}}\left(\bar{\alpha}_{r^{\prime}}^{\prime}, \bar{\alpha}_{r^{\prime \prime}}^{\prime \prime}, \bar{e}_{i}\right) \bar{e}_{i}^{*} r^{\prime} r^{\prime \prime} q^{\lambda},
$$

where the $\bar{e}_{i}$ form a basis of $H^{*}(\bar{M})$ and the $\bar{e}_{i}^{*}$ denote the dual basis with respect to the cup product pairing (see [13]).

Corollary 6.2. Let $\tau:=\sum_{v=1}^{n} \mathrm{w}_{v}$, suppose that $T$ acts freely on $\mu^{-1}(\tau)$, and assume that the minimal Chern number $N$ of $\bar{M}$ is greater that one. Then

$$
\overline{\mathrm{w}}^{* d^{+}}=\overline{\mathrm{w}}^{* d^{-}} q^{\lambda_{d}}
$$

for every $d \in \mathcal{D}_{\text {eff }}(\tau)$.

Proof. By the gluing theorem for the Gromov-Witten invariants [13], we have

$$
\begin{aligned}
\overline{\mathrm{w}}^{* d^{+}} & =\sum_{i, \lambda} \mathrm{GW}_{\bar{\lambda}}^{\bar{M}}\left(\overline{\mathrm{w}}^{* d^{+}}, \bar{e}_{i}\right) \bar{e}_{i}^{*} q^{\lambda} \\
& =\sum_{i, \lambda} \mathrm{GW}_{\bar{\lambda}-\bar{\lambda}_{d}}^{\bar{M}}\left(\overline{\mathrm{w}}^{* d^{-}}, \bar{e}_{i}\right) \bar{e}_{i}^{*} q^{\lambda} \\
& =\overline{\mathrm{w}}^{* d^{-}} q^{\lambda_{d}} .
\end{aligned}
$$

The second equality follows from Lemma 6.1.

Proof of Theorem 1.3. We prove that the homomorphism (4) is surjective. Note that there is an obvious inclusion $H^{*}(\bar{M}) \rightarrow \mathrm{QH}^{*}(\bar{M} ; \mathcal{R}): \bar{\alpha} \mapsto \bar{\alpha} 1$, where 1 denotes the unit in $\mathcal{R}$. Throughout we identify $H^{*}(\bar{M})$ with its image in $\mathrm{QH}^{*}(\bar{M} ; \mathcal{R})$ under this homomorphism. Since (4) is a homomorphism of $\mathcal{R}$-modules, it suffices to prove that every class in $H^{*}(\bar{M})$ belongs to the image of (4). We prove this by induction over the degree. If $\bar{\alpha} \in H^{0}(\bar{M})$ then $\bar{\alpha}$ obviously belongs to the image of (4). Hence let $\operatorname{deg}(\bar{\alpha})=2 \ell>0$ and assume, by induction, that every class in $H^{*}(\bar{M})$ of degree less than $2 \ell$ belongs to the image of (4). By Kirwan's theorem, the class $\bar{\alpha}$ is a linear combination of classes of the form $\overline{\mathrm{w}}_{\nu_{1}} \cdots \overline{\mathrm{w}}_{\nu_{\ell}}$. Let $p\left(u_{1}, \ldots, u_{n}\right)$ be the same linear combination of the polynomials $u_{v_{1}} \cdots u_{\nu_{\ell}}$. Then the image of $p(u)$ under the homomorphism differs from $\bar{\alpha}$ by a class of the form

$$
\bar{\beta}=\sum_{\lambda \neq 0} \bar{\beta}_{\lambda} q^{\lambda}, \quad \operatorname{deg}\left(\bar{\beta}_{\lambda}\right)=2 \ell-2\langle\tau, \lambda\rangle<2 \ell .
$$

Here the sum is over all $\lambda \in \Lambda_{\text {eff }}(\tau)$ that satisfy $\langle\tau, \lambda\rangle>0$. Hence, by the induction hypothesis, every $\bar{\beta}_{\lambda}$ in this sum belongs to the image of (4), and so does the class $\bar{\beta}_{\lambda} q^{\lambda}$. Hence $\bar{\beta}$ belongs to the image of (4), and so does $\bar{\alpha}$. 
Let $\mathcal{J}_{0} \subset \mathcal{R}\left[u_{1}, \ldots, u_{n}\right]$ be the kernel of (4). Then the linear polynomial $\sum_{\nu} \eta_{\nu} u_{v}$ belongs to $\mathcal{J}_{0}$ whenever $\sum_{\nu} \eta_{\nu} \mathrm{w}_{\nu}=0$. Moreover, by Corollary 6.2, the polynomial $u^{d^{+}}-q^{\lambda} u^{d^{-}}$belongs to $\mathcal{J}_{0}$ whenever $\lambda \in \Lambda_{\text {eff }}(\tau)$ and $d_{v}^{ \pm}$ $=\max \left\{ \pm\left\langle\mathrm{w}_{\nu}, \lambda\right\rangle, 0\right\}$. Hence $\mathcal{J} \subset \mathcal{J}_{0}$.

We prove that $\mathcal{J}_{0} \subset \mathcal{J}$. Define the classes $\bar{\alpha}_{\ell, \lambda} \in H^{*}(\bar{M})$, for $n$-tuples $\ell$ of nonnegative integers and lattice vectors $\lambda \in \Lambda_{\text {eff }}(\tau)$ with $0<\langle\tau, \lambda\rangle \leq|\ell|$, by

$$
\overline{\mathrm{w}}^{* \ell}=: \overline{\mathrm{w}}^{\ell}+\sum_{\lambda \in \Lambda_{\mathrm{eff}}(\tau) \backslash\{0\}} \bar{\alpha}_{\ell, \lambda} q^{\lambda}, \quad \operatorname{deg}\left(\bar{\alpha}_{\ell, \lambda}\right)=2|\ell|-2\langle\tau, \lambda\rangle .
$$

For $N \in \mathbb{Z}$ denote by $\mathcal{J}_{0}(N)$ the set of polynomials $p \in \mathcal{J}_{0}$ of the form

$$
p\left(u_{1}, \ldots, u_{n}\right)=\sum_{|\ell| \leq N} r_{\ell} u^{\ell},
$$

where the sum is over all $n$-tuples $\ell=\left(\ell_{1}, \ldots, \ell_{n}\right)$ of nonnegative integers satisfying $|\ell| \leq N$. We prove by induction on $N$ that $\mathcal{J}_{0}(N) \subset \mathcal{J}$. For $N<0$ this is obvious because $\mathcal{J}_{0}(N)=\{0\}$. Let $N \geq 0$ and assume by induction that $\mathcal{J}_{0}(N-1) \subset \mathcal{J}$. Let $p \in \mathcal{J}_{0}(N)$ be a polynomial of the form (48). Since $p \in \mathcal{J}_{0}$ we have

$$
0=\sum_{\ell} r_{\ell} \overline{\mathrm{w}}^{* \ell}=\sum_{\ell} \overline{\mathrm{w}}^{\ell} r_{\ell}+\sum_{\ell} \sum_{\lambda} \bar{\alpha}_{\ell, \lambda} r_{\ell} q^{\lambda} .
$$

This identity splits up into

$$
0=\sum_{|\ell|=j} r_{\ell} \overline{\mathrm{w}}^{\ell}+\sum_{|\ell|>j} \sum_{\substack{\lambda \tau, \lambda\rangle=|\ell|-j \\\langle}} \bar{\alpha}_{\ell, \lambda} r_{\ell} q^{\lambda}, \quad j=0, \ldots, N .
$$

Since $r_{\ell}=0$ for $|\ell|>N$, we have

$$
\sum_{|\ell|=N} r_{\ell} \overline{\mathrm{w}}^{\ell}=0
$$

Choose a basis $\rho_{1}, \ldots, \rho_{m}$ of the vector space $\operatorname{span}\left\{r_{\ell}|| \ell \mid=N\right\} \subset \mathcal{R}$ and express each $r_{\ell}$ in this basis, i.e.

$$
r_{\ell}=\sum_{i=1}^{m} a_{\ell i} \rho_{i}, \quad a_{\ell i} \in \mathbb{R}, \quad|\ell|=N .
$$

Then

$$
\sum_{|\ell|=N} a_{\ell i} \overline{\mathrm{w}}^{\ell}=0, \quad i=1, \ldots, m .
$$

This means that the polynomials

$$
p_{i 0}\left(u_{1}, \ldots, u_{n}\right):=\sum_{|\ell|=N} a_{\ell i} u^{\ell}, \quad i=1, \ldots, m,
$$


belong to the kernel $\mathcal{I} \subset \mathbb{R}\left[u_{1}, \ldots, u_{n}\right]$ of the homomorphism (59) in Theorem E.4. Hence they can be expressed in the form

$$
p_{i 0}=\sum_{j} p_{i j 0} f_{j}
$$

where $f_{j} \in \mathbb{R}\left[u_{1}, \ldots, u_{n}\right]$, and the $p_{i j 0}$ are taken from the set of generators of $\mathcal{I}$ in Theorem E.5. Thus each $p_{i j 0}$ satisfies one of the following conditions.

(a) $p_{i j 0}(u)=\sum_{\nu} \eta_{\nu} u_{v}$, where $\sum_{v} \eta_{\nu} \mathrm{w}_{v}=0$.

(b) $p_{i j 0}(u)=u_{v}$, where $\Delta_{\{v\}}=\emptyset$.

(c) $p_{i j 0}(u)=u^{d^{+}}$, where $d \in \mathcal{D}_{\text {eff }}(\tau) \backslash\{0\}$.

In cases (a) and (b) define $p_{i j}:=p_{i j 0} \in \mathcal{J}$. In the case (c) it follows from the definition of $\mathcal{J}$ that there is a generator $p_{i j} \in \mathcal{J}$ of the form

$$
p_{i j}(u)=u^{d^{+}}-q^{\lambda} u^{d^{-}}, \quad p_{i j 0}(u)=u^{d^{+}}, \quad d \in \mathcal{D}_{\text {eff }}(\tau) \backslash\{0\} .
$$

Define $\hat{p} \in \mathcal{J}$ by

$$
\hat{p}(u):=\sum_{i=1}^{m} \sum_{j} \rho_{i} p_{i j}(u) f_{j}(u) .
$$

Since $\mathcal{J} \subset \mathcal{J}_{0}$ we have $p-\hat{p} \in \mathcal{J}_{0}$. Since

$$
\sum_{i=1}^{m} \sum_{j} \rho_{i} p_{i j 0}(u) f_{j}(u)=\sum_{i=1}^{m} \rho_{i} p_{i 0}(u)=\sum_{i=1}^{m} \sum_{|\ell|=N} \rho_{i} a_{\ell i} u^{\ell}=\sum_{|\ell|=N} r_{\ell} u^{\ell},
$$

the leading terms cancel in $p-\hat{p}$ and hence $p-\hat{p} \in \mathcal{J}_{0}(N-1) \subset \mathcal{J}$. Hence $p \in \mathcal{J}$. This completes the induction and the proof of the theorem.

Example 6.3. This example shows that in the definition of the ideal $\mathcal{J}$ it may not suffice to consider vectors $\lambda \in \Lambda_{\text {eff }}(\tau)$ such that the integers $d_{v}:=\left\langle\mathrm{w}_{v}, \lambda\right\rangle$ are all nonnegative. Suppose the 2-torus $T=\mathbb{T}^{2}$ acts on $\mathbb{C}^{5}$ with weight vectors

$$
\mathrm{w}_{1}=(1,0), \quad \mathrm{w}_{2}=(1,1), \quad \mathrm{w}_{3}=\mathrm{w}_{4}=\mathrm{w}_{5}=(0,1) .
$$

The symplectic quotient $\bar{M}$ at the parameter $\tau:=\mathrm{w}_{1}+\cdots+\mathrm{w}_{5}=(2,4)$ is a smooth monotone toric 3-fold with minimal Chern number $N=2$. The effective cone is given by

$$
\Lambda_{\text {eff }}(\tau)=\left\{\left(\lambda_{1}, \lambda_{2}\right) \in \mathbb{Z}^{2} \mid \lambda_{2} \geq 0, \lambda_{1}+\lambda_{2} \geq 0\right\} .
$$

It is the convex cone spanned by the vectors $e:=(1,0)$ and $e^{\prime}:=(-1,1)$ with $d=(1,1,0,0,0)$ and $d^{\prime}=(-1,0,1,1,1)$. For the quantum cohomology let us choose the polynomial ring $\mathcal{R}:=\mathbb{R}\left[q_{1}, q_{2}\right]$, graded by $\operatorname{deg}\left(q_{1}\right)=\operatorname{deg}\left(q_{2}\right)=4$, 
and the homomorphism $q^{\lambda}:=q_{1}^{\lambda_{1}+\lambda_{2}} q_{2}^{\lambda_{2}}$. Thus $q_{1}, q_{2}$ correspond to the generators $e, e^{\prime}$ of $\Lambda_{\text {eff }}(\tau)$. Then the ideal $\mathcal{J} \subset \mathbb{R}\left[u_{1}, \ldots, u_{5}, q_{1}, q_{2}\right]$ is generated by the relations

$$
u_{3}=u_{4}=u_{5}=u_{2}-u_{1}, \quad u_{1} u_{2}=q_{1}, \quad u_{3} u_{4} u_{5}=u_{1} q_{2} .
$$

If one considers only vectors $\lambda \in \Lambda_{\text {eff }}(\tau)$ with nonnegative degrees $d_{\nu}$ $:=\left\langle\mathrm{w}_{\nu}, \lambda\right\rangle$ then one has to replace the last relation in (49) by $u_{2} u_{3} u_{4} u_{5}=q_{1} q_{2}$ and obtains a strictly smaller ideal.

\section{Appendix A. G-moduli problems}

In this appendix we summarize results from [8] that are used in this paper.

Definition A.1. Let $\mathrm{G}$ be a compact Lie group. A G-moduli problem is a triple $(\mathcal{B}, \mathcal{E}, \mathcal{S})$ with the following properties.

- $\mathcal{B}$ is a Hilbert manifold (without boundary) equipped with a smooth G-action.

- $\mathcal{E}$ is a Hilbert space bundle over $\mathcal{B}$, also equipped with a smooth G-action, such that $\mathrm{G}$ acts by isometries on the fibres of $\mathcal{E}$ and the projection $\mathcal{E} \rightarrow \mathcal{B}$ is G-equivariant.

- $\mathcal{S}: \mathcal{B} \rightarrow \mathcal{E}$ is a smooth G-equivariant Fredholm section of constant Fredholm index such that the determinant bundle $\operatorname{det}(\mathcal{S}) \rightarrow \mathcal{B}$ is oriented, $\mathrm{G}$ acts by orientation preserving isomorphisms on the determinant bundle, and the zero set

$$
\mathcal{M}:=\{x \in \mathcal{B} \mid \mathcal{S}(x)=0\}
$$

is compact.

A G-moduli problem $(\mathcal{B}, \mathcal{E}, \mathcal{S})$ is called regular if the isotropy subgroup $\mathrm{G}_{x}:=\left\{g \in \mathrm{G} \mid g^{*} x=x\right\}$ is finite for every $x \in \mathcal{M}$. A finite dimensional regular G-moduli problem $(B, E, S)$ is called oriented if $T B / \mathfrak{g}$ and $E$ are oriented vector bundles over $B$ and $\mathrm{G}$ acts on both bundles by orientation preserving diffeomorphisms.

Let $(\mathcal{B}, \mathcal{E}, \mathcal{S})$ be a G-moduli problem. The fibre of $\mathcal{E}$ over $x \in \mathcal{B}$ will be denoted by $\mathcal{E}_{x}$. Thus elements of $\mathcal{E}$ are pairs $(x, e)$, where $x \in \mathcal{B}$ and $e \in \mathcal{E}_{x}$. In this notation a section is a map of the form $\mathcal{B} \rightarrow \mathcal{E}: x \mapsto(x, \mathcal{S}(x))$, where $\mathcal{S}(x) \in \mathcal{E}_{x}$. Abusing notation, we also denote the map $\mathcal{B} \rightarrow \mathcal{E}$ by $\mathcal{S}$. The Fredholm property asserts that, for $x \in \mathcal{M}=\mathcal{S}^{-1}(0)$, the vertical differential

$$
\mathcal{D}_{x}:=D \mathcal{S}(x): T_{x} \mathcal{B} \rightarrow \mathcal{E}_{x}
$$

is a Fredholm operator whose Fredholm index is independent of $x$. Since $\mathcal{S}$ is equivariant there is a complex

$$
0 \longrightarrow \mathfrak{g} \stackrel{L_{x}}{\longrightarrow} T_{x} \mathcal{B} \stackrel{\mathcal{D}_{x}}{\longrightarrow} \mathcal{E}_{x} \longrightarrow 0,
$$


where the map $L_{x}: \mathfrak{g} \rightarrow T_{x} \mathcal{B}$ is the infinitesimal action. Note that the index of this complex is

$$
\operatorname{index}(\mathcal{S}):=\operatorname{index}\left(\mathcal{D}_{x}\right)-\operatorname{dim} \mathrm{G} .
$$

Its determinant line is defined by

$$
\operatorname{det}(\mathcal{S})_{x}:=\Lambda^{\max }\left(\operatorname{ker} L_{x}\right)^{*} \otimes \Lambda^{\max }\left(\operatorname{ker} \mathcal{D}_{x} / \operatorname{im} L_{x}\right) \otimes \Lambda^{\max }\left(\mathcal{E}_{x} / \operatorname{im} \mathcal{D}_{x}\right)^{*} .
$$

This defines a real line bundle over $\mathcal{M}$ which extends to a line bundle over an open neighbourhood of $\mathcal{M}$. The orientation hypothesis asserts that the determinant bundle is oriented over such a neighbourhood. Note that in the finite dimensional regular case $\operatorname{det}(S)_{x} \cong \Lambda^{\max }\left(T_{x} B / \operatorname{im} L_{x}\right) \otimes \Lambda^{\max }\left(E_{x}\right)^{*}$ and the orientation hypothesis asserts that both factors are oriented. G-moduli problems form a category as follows.

Definition A.2. Let $(\mathcal{B}, \mathcal{E}, \mathcal{S}),\left(\mathcal{B}^{\prime}, \mathcal{E}^{\prime}, \mathcal{S}^{\prime}\right)$ be G-moduli problems. A morphism from $(\mathcal{B}, \mathcal{E}, \mathcal{S})$ to $\left(\mathcal{B}^{\prime}, \mathcal{E}^{\prime}, \mathcal{S}^{\prime}\right)$ is a pair $(\psi, \Psi)$ with the following properties.

$$
\psi: \mathcal{B}_{0} \rightarrow \mathcal{B}^{\prime}
$$

is a smooth G-equivariant embedding of a neighbourhood $\mathcal{B}_{0} \subset \mathcal{B}$ of $\mathcal{M}$ into $\mathcal{B}^{\prime}$,

$$
\Psi: \mathcal{E}_{0}:=\left.\mathcal{E}\right|_{\mathcal{B}_{0}} \rightarrow \mathcal{E}^{\prime}
$$

is a smooth injective bundle homomorphism and a lift of $\psi$, and the sections $\mathcal{S}$ and $\mathcal{S}^{\prime}$ satisfy

$$
\mathcal{S}^{\prime} \circ \psi=\Psi \circ \mathcal{S}, \quad \mathcal{M}^{\prime}=\psi(\mathcal{M}) .
$$

Moreover, the linear operators $d_{x} \psi: T_{x} \mathcal{B} \rightarrow T_{\psi(x)} \mathcal{B}^{\prime}$ and $\Psi_{x}: \mathcal{E}_{x} \rightarrow \mathcal{E}_{\psi(x)}^{\prime}$ induce isomorphisms

$$
d_{x} \psi: \operatorname{ker} \mathcal{D}_{x} \rightarrow \operatorname{ker} \mathcal{D}_{\psi(x)}^{\prime}, \quad \Psi_{x}: \operatorname{coker} \mathcal{D}_{x} \rightarrow \operatorname{coker} \mathcal{D}_{\psi(x)}^{\prime},
$$

for $x \in \mathcal{M}$, and the resulting isomorphism from $\operatorname{det}(\mathcal{S})$ to $\operatorname{det}\left(\mathcal{S}^{\prime}\right)$ is orientation preserving.

Let $(\mathcal{B}, \mathcal{E}, \mathcal{S})$ and $\left(\mathcal{B}^{\prime}, \mathcal{E}^{\prime}, \mathcal{S}^{\prime}\right)$ be G-moduli problems and suppose that there exists a morphism from $(\mathcal{B}, \mathcal{E}, \mathcal{S})$ to $\left(\mathcal{B}^{\prime}, \mathcal{E}^{\prime}, \mathcal{S}^{\prime}\right)$. Then the indices of $\mathcal{S}$ and $\mathcal{S}^{\prime}$ agree. Moreover, $(\mathcal{B}, \mathcal{E}, \mathcal{S})$ is regular if and only if $\left(\mathcal{B}^{\prime}, \mathcal{E}^{\prime}, \mathcal{S}^{\prime}\right)$ is regular.

Definition A.3. Two regular G-moduli problems $\left(\mathcal{B}_{i}, \mathcal{E}_{i}, \mathcal{S}_{i}\right), i=0,1$, are called cobordant if there exist a G-equivariant Hilbert space bundle $\widetilde{\mathcal{E}} \rightarrow \widetilde{\mathcal{B}}$ over a Hilbert manifold $\widetilde{\mathcal{B}}$ with boundary, a smooth oriented G-equivariant Fredholm section $\widetilde{\mathcal{S}}: \widetilde{\mathcal{B}} \rightarrow \widetilde{\mathcal{E}}$ such that the zero set $\widetilde{\mathcal{M}}:=\widetilde{\mathcal{S}}^{-1}(0)$ is compact, $\mathrm{G}$ acts with finite isotropy on $\widetilde{\mathcal{B}}$, and

$$
\partial \widetilde{\mathcal{B}}=\mathcal{B}_{0} \cup \mathcal{B}_{1}, \quad \mathcal{E}_{i}=\left.\widetilde{\mathcal{E}}\right|_{\mathcal{B}_{i}}, \quad \mathcal{S}_{i}=\left.\widetilde{\mathcal{S}}\right|_{\mathcal{B}_{i}}
$$


Moreover, $\operatorname{det}(\widetilde{\mathcal{S}})$ carries an orientation which induces the orientation of $\operatorname{det}\left(\mathcal{S}_{1}\right)$ over $\mathcal{B}_{1}$ and the opposite of the orientation of $\operatorname{det}\left(\mathcal{S}_{0}\right)$ over $\mathcal{B}_{0}$. Here an orientation of $\operatorname{det}(\widetilde{\mathcal{S}})$ induces an orientation of the determinant bundle of $\mathcal{S}:=\left.\widetilde{\mathcal{S}}\right|_{\partial \widetilde{\mathcal{B}}}$ via the natural isomorphism $\left.\operatorname{det}(\widetilde{\mathcal{S}})\right|_{\partial \widetilde{\mathcal{B}}} \cong \mathbb{R} v \otimes \operatorname{det}(\mathcal{S})$ for an outward pointing normal vector field $v$ along $\partial \widetilde{\mathcal{B}}$.

The next theorem is proved in [8]. It states the properties of the Euler class. We denote by $H_{\mathrm{G}}^{*}(\mathcal{B})$ the equivariant cohomology with real coefficients.

Theorem A.4. There exists a functor, called the Euler class, which assigns to each compact Lie group $\mathrm{G}$ and each regular $\mathrm{G}$-moduli problem $(\mathcal{B}, \mathcal{E}, \mathcal{S})$ a homomorphism $\chi^{\mathcal{B}, \mathcal{E}, \mathcal{S}}: H_{\mathrm{G}}^{*}(\mathcal{B}) \rightarrow \mathbb{R}$ and satisfies the following.

(Functoriality) If $(\psi, \Psi)$ is a morphism from $(\mathcal{B}, \mathcal{E}, \mathcal{S})$ to $\left(\mathcal{B}^{\prime}, \mathcal{E}^{\prime}, \mathcal{S}^{\prime}\right)$ then $\chi^{\mathcal{B}, \mathcal{E}, \mathcal{S}}$ $\left(\psi^{*} \alpha\right)=\chi^{\mathcal{B}^{\prime}, \mathcal{E}^{\prime}, \mathcal{S}^{\prime}}(\alpha)$ for every $\alpha \in H_{\mathrm{G}}^{*}\left(\mathcal{B}^{\prime}\right)$.

(Thom class) If $(B, E, S)$ is a finite dimensional oriented regular G-moduli problem and $\tau \in \Omega_{\mathrm{G}}^{*}(E)$ is an equivariant Thom form supported in an open neighbourhood $U \subset E$ of the zero section such that $U \cap E_{x}$ is convex for every $x \in B, U \cap \pi^{-1}(K)$ has compact closure for every compact set $K \subset B$, and $S^{-1}(U)$ has compact closure, then

$$
\chi^{B, E, S}(\alpha)=\int_{B / G} \alpha \wedge S^{*} \tau
$$

for every $\alpha \in H_{\mathrm{G}}^{*}(B)$.

(Transversality) If $\mathcal{S}$ is transverse to the zero section then

$$
\chi^{\mathcal{B}, \mathcal{E}, \mathcal{S}}(\alpha)=\int_{\mathcal{M} / \mathrm{G}} \alpha
$$

for every $\alpha \in H_{\mathrm{G}}^{*}(B)$, where $\mathcal{M}:=\mathcal{S}^{-1}(0)$.

(Cobordism) If $\left(\mathcal{B}_{0}, \mathcal{E}_{0}, \mathcal{S}_{0}\right),\left(\mathcal{B}_{1}, \mathcal{E}_{1}, \mathcal{S}_{1}\right)$ are cobordant $\mathrm{G}$-moduli problems then

$$
\chi^{\mathcal{B}_{0}, \mathcal{E}_{0}, \mathcal{S}_{0}}\left(\iota_{0}^{*} \alpha\right)=\chi^{\mathcal{B}_{0}, \mathcal{E}_{1}, \mathcal{S}_{1}}\left(\iota_{1}^{*} \alpha\right)
$$

for every $\alpha \in H_{\mathrm{G}}^{*}(\widetilde{\mathcal{B}})$, where $\iota_{0}: \mathcal{B}_{0} \hookrightarrow \widetilde{\mathcal{B}}$ and $\iota_{1}: \mathcal{B}_{1} \hookrightarrow \widetilde{\mathcal{B}}$ are the inclusions.

The Euler class is uniquely determined by the (Functoriality) and (Thom class) axioms.

The integrals in the (Transversality) and (Thom class) axioms are defined in terms of local slices for the G-action and an equivariant partition of unity. For details see [8]. 


\section{Appendix B. Determinants}

Let $X$ and $Y$ be real Banach spaces and denote by $\mathcal{F}(X, Y)$ the space of linear Fredholm operators $D: X \rightarrow Y$. For every nonnegative integer $m$ denote by $\mathcal{L}\left(X, \mathbb{R}^{m}\right)$ the space of bounded linear operators $\Phi: X \rightarrow \mathbb{R}^{m}$. If $D \in \mathcal{F}(X, Y)$ and $\Phi \in \mathcal{L}\left(X, \mathbb{R}^{m}\right)$ then the operator $D \oplus \Phi: X \rightarrow Y \oplus \mathbb{R}^{m}$, defined by

$$
(D \oplus \Phi) x:=(D x, \Phi x),
$$

is Fredholm and index $(D \oplus \Phi)=\operatorname{index}(D)-m$. The determinant line of a Fredholm operator $D \in \mathcal{F}(X, Y)$ is defined by

$$
\operatorname{det}(D):=\Lambda^{\max } \operatorname{ker} D \otimes \Lambda^{\max } \operatorname{ker} D^{*} .
$$

Lemma B.1. There is a family of isomorphisms

$$
T(D, \Phi): \operatorname{det}(D) \rightarrow \operatorname{det}(D \oplus \Phi),
$$

one for nonnegative integer $n$ and each pair $(D, \Phi) \in \mathcal{F}(X, Y) \times \mathcal{L}\left(X, \mathbb{R}^{m}\right)$, such that

$$
T(D, \Phi \oplus \Psi)=T(D \oplus \Phi, \Psi) \circ T(D, \Phi)
$$

for $D \in \mathcal{F}(X, Y), \Phi \in \mathcal{L}\left(X, \mathbb{R}^{m}\right)$, and $\Psi \in \mathcal{L}\left(X, \mathbb{R}^{n}\right)$.

Proof. There is an obvious isomorphism

$$
\left(\frac{\operatorname{ker} D}{\operatorname{ker} D \cap \operatorname{ker} \Phi}\right)^{*} \cong \frac{\operatorname{im} \Phi^{*}}{\operatorname{im} D^{*} \cap \operatorname{im} \Phi^{*}} .
$$

Moreover,

$$
\operatorname{ker}(D \oplus \Phi)=\operatorname{ker} D \cap \operatorname{ker} \Phi \subset \operatorname{ker} D
$$

and hence

$$
\Lambda^{\max } \operatorname{ker} D \cong \Lambda^{\max } \operatorname{ker}(D \oplus \Phi) \otimes \Lambda^{\max }\left(\frac{\operatorname{ker} D}{\operatorname{ker} D \cap \operatorname{ker} \Phi}\right) .
$$

Now the kernel of $(D \oplus \Phi)^{*}$ is given by

$$
\operatorname{ker}(D \oplus \Phi)^{*}=\left\{\left(y^{*}, z^{*}\right) \in Y^{*} \oplus\left(\mathbb{R}^{m}\right)^{*} \mid D^{*} y^{*}+\Phi^{*} z^{*}=0\right\} .
$$

So there is an exact sequence

$$
0 \rightarrow \operatorname{ker} D^{*} \oplus \operatorname{ker} \Phi^{*} \rightarrow \operatorname{ker}(D \oplus \Phi)^{*} \rightarrow \operatorname{im} D^{*} \cap \operatorname{im} \Phi^{*} \rightarrow 0 .
$$

Here the second map is the obvious inclusion and the third map is given by $\left(y^{*}, z^{*}\right) \mapsto D^{*} y^{*}=-\Phi^{*} z^{*}$. This shows that there is an isomorphism

$$
\Lambda^{\max } \operatorname{ker} D^{*} \otimes \Lambda^{\max } \operatorname{ker} \Phi^{*} \otimes \Lambda^{\max }\left(\operatorname{im} D^{*} \cap \operatorname{im} \Phi^{*}\right) \cong \Lambda^{\max } \operatorname{ker}(D \oplus \Phi)^{*} .
$$


Moreover,

$$
\Lambda^{\max } \operatorname{ker} \Phi^{*} \otimes \Lambda^{\max } \operatorname{im} \Phi^{*} \cong \Lambda^{\max } \operatorname{ker} \Phi^{*} \otimes \Lambda^{\max }\left(\frac{\left(R^{m}\right)^{*}}{\operatorname{ker} \Phi^{*}}\right) \cong \mathbb{R}
$$

Here the first isomorphism is induced by the isomorphism

$$
\Phi^{*}:\left(\mathbb{R}^{m}\right)^{*} / \operatorname{ker} \Phi^{*} \rightarrow \operatorname{im} \Phi^{*}
$$

and the second one by the canonical orientation of $\mathbb{R}^{m}$. Combinig the last two isomorphisms, we obtain

$$
\Lambda^{\max } \operatorname{ker} D^{*} \otimes \Lambda^{\max }\left(\operatorname{im} D^{*} \cap \operatorname{im} \Phi^{*}\right) \cong \Lambda^{\max } \operatorname{ker}(D \oplus \Phi)^{*} \otimes \Lambda^{\max } \operatorname{im} \Phi^{*},
$$

and hence

$$
\Lambda^{\max } \operatorname{ker} D^{*} \cong \Lambda^{\max } \operatorname{ker}(D \oplus \Phi)^{*} \otimes \Lambda^{\max }\left(\frac{\operatorname{im} \Phi^{*}}{\operatorname{im} D^{*} \cap \operatorname{im} \Phi^{*}}\right) .
$$

Combining the isomorphisms (54) and (55), and using (53), we obtain the required isomorphism $T(D, \Phi): \operatorname{det}(D) \rightarrow \operatorname{det}(D \oplus \Phi)$. The construction shows that these isomorphisms satisfy equation (52).

Remark B.2. If $X$ and $Y$ are complex Banach spaces and $D \in \mathcal{F}(X, Y)$ and $\Phi \in \mathcal{L}\left(X, \mathbb{C}^{m}\right)$ are complex linear then the isomorphism $T(D, \Phi): \operatorname{det}(D) \rightarrow$ $\operatorname{det}(D \oplus \Phi)$ of Lemma B.1 preserves the orientations arising from the complex structures. This implies that the determinant line bundle over the space of complex linear Fredholm operators carries a canonical orientation.

Let $D \in \mathcal{F}(X, Y), \Phi \in \mathcal{L}\left(X, \mathbb{R}^{m}\right)$, and $\Psi: \mathbb{R}^{m} \rightarrow \mathbb{R}^{m}$ be an isomorphism. Then the kernels of $D \oplus \Phi$ and $D \oplus \Psi \Phi$ are equal and there is an isomorphism $\operatorname{ker}(D \oplus \Psi \Phi)^{*} \rightarrow \operatorname{ker}(D \oplus \Phi)^{*}:\left(y^{*}, z^{*}\right) \mapsto\left(y^{*}, \Psi^{*} z^{*}\right)$. This induces an isomorphism

$$
U(\Psi): \operatorname{det}(D \oplus \Psi \Phi) \rightarrow \operatorname{det}(D \oplus \Phi)
$$

Lemma B.3. For every $D \in \mathcal{F}(X, Y)$, every $\Phi \in \mathcal{L}\left(X, \mathbb{R}^{m}\right)$ and every isomorphism $\Psi: \mathbb{R}^{m} \rightarrow \mathbb{R}^{m}$ the following diagram commutes:

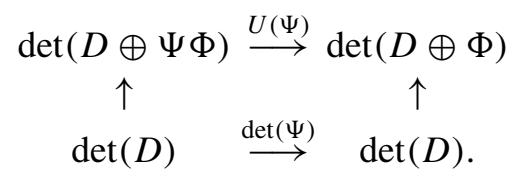

Here the vertical maps are $T(D, \Psi \Phi)$ and $T(D, \Phi)$, respectively. 
Proof. We prove that there is a commutative diagram

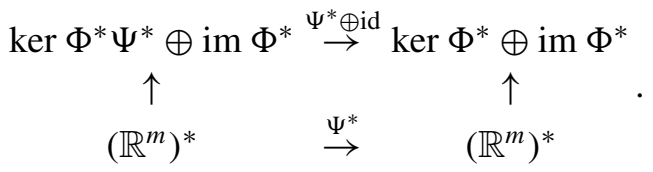

To see this, choose a decomposition

$$
\left(\mathbb{R}^{m}\right)^{*} \cong \operatorname{ker}\left(\Phi^{*} \Psi^{*}\right) \oplus W,
$$

and observe that

$$
\left(\mathbb{R}^{m}\right)^{*} \cong \operatorname{ker} \Phi^{*} \oplus \Psi^{*} W .
$$

With respect to this decomposition, the left vertical arrow in (56) is given by $(v, w) \mapsto\left(v, \Phi^{*} \Psi^{*} w\right)$, and similarly for the right vertical arrow. Note that although these maps depend on the choice of $W$, the induced maps between the top exterior powers are independent of this choice. Commutativity of the diagram (56) is now obvious from the definition of the maps. The result follows from (56) by taking top exterior powers and observing how the resulting maps fit into the construction of the isomorphisms $T(D, \Phi)$ and $T(D, \Psi \Phi)$.

Consider now Banach spaces $X, Z, V$ and bounded linear operators

$$
F: X \rightarrow Z, \quad K: X \rightarrow V
$$

such that $K$ is surjective.

Lemma B.4. The operator $\left.F\right|_{\mathrm{ker} K}$ is Fredholm if and only if the augmented operator $F \oplus K: X \rightarrow Z \oplus V$ is, and in this case their Fredholm indices agree. Moreover, there is a collection of isomorphisms

$$
S(F, K): \operatorname{det}\left(\left.F\right|_{\text {ker } K}\right) \rightarrow \operatorname{det}(F \oplus K),
$$

one for every such pair $F, K$, such that for every $\Phi \in \mathcal{L}\left(X, \mathbb{R}^{m}\right)$ the following diagram commutes:

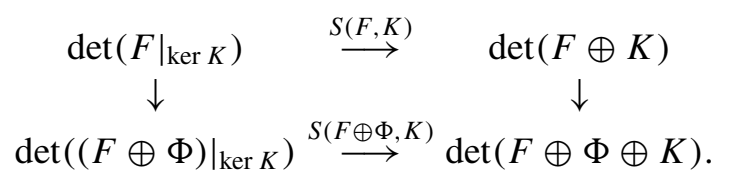

Here the vertical maps are $T\left(\left.F\right|_{\operatorname{ker} K},\left.\Phi\right|_{\operatorname{ker} K}\right)$ and $T(F \oplus K, \Phi)$, respectively.

Proof. There are obvious isomorphisms

$$
\operatorname{ker}\left(\left.F\right|_{\text {ker } K}\right) \rightarrow \operatorname{ker}(F \oplus K), \quad \operatorname{coker}\left(\left.F\right|_{\text {ker } K}\right) \rightarrow \operatorname{coker}(F \oplus K) .
$$

These induce the required isomorphism $S(F, K)$. 
The proof of the next lemma is straightforward.

Lemma B.5. Let $F$ and $K$ be as in Lemma B.4 and and $\Psi: V \rightarrow V^{\prime}$ be a Banach space isomorphism. Then the following diagram commutes:

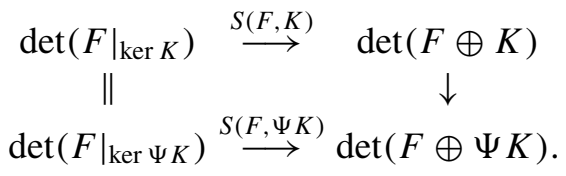

Here the right vertical arrow is induced by the map id $\oplus \Psi: Z \oplus V \rightarrow Z \oplus V^{\prime}$. (The kernels are equal.)

\section{Appendix C. Equivariant trivialization}

Proposition C.1. Let $\mathrm{G}$ be a compact Lie group and $E \rightarrow X$ be a $\mathrm{G}$-equivariant complex vector bundle over compact smooth manifold $X$. Then there exists a G-equivariant complex vector bundle $F \rightarrow X$ and a complex G-representation $W$ such that $E \oplus F$ is equivariantly isomorphic to $X \times W$.

Lemma C.2. Let $\mathrm{G}$ be a compact Lie group, $\mathrm{H} \subset \mathrm{G}$ be a normal subgroup, and $V$ be a complex $\mathrm{H}-$ representation. Then there exists a complex $\mathrm{G}-$ representation $W$ and an injective $\mathrm{H}-$ equivariant homorphism $\phi: V \rightarrow W$.

Proof. Consider the infinite dimensional vector space

$$
\mathcal{W}:=\{f: \mathrm{G} \rightarrow V \text { continuous } \mid f(h g)=h f(g) \forall h \in \mathrm{H}, \forall g \in \mathrm{G}\} .
$$

This space carries an action of $\mathrm{G}$ by

$$
\left(g^{\prime} f\right)(g):=f\left(g g^{\prime}\right)
$$

and the evaluation map $\mathcal{W} \rightarrow V: f \mapsto f(1)$ is H-equivariant and surjective. To prove surjectivity let $v \in V$ be given and let $f: \mathrm{G} \rightarrow V$ be any continuous extension of the map $\mathrm{H} \rightarrow V: h \mapsto h v$. By averaging the maps $g \mapsto h^{-1} f(h g)$ over $h \in \mathrm{H}$ we can ensure that the extension is H-equivariant. By Peter-Weyl's theorem, there exists a finite dimensional G-invariant subspace $W \subset \mathcal{W}$ such that the restriction of the homomorphism $f \mapsto f(1)$ to $W$ is still surjective. By [13, Remark A.4.2], the surjection $W \rightarrow V$ has an H-equivariant right inverse.

Proof of Proposition C.1. Let $x \in X, \mathrm{H} \subset \mathrm{G}$ be the isotropy subgroup of $x$, and $V:=E_{x}$. By the local slice theorem, the restriction of $E$ to a suitable neighbourhood of the G-orbit of $x$ is equivariantly isomorphic to the bundle

$$
\frac{\mathrm{G} \times U \times V}{\mathrm{H}} \rightarrow \mathrm{G} \times_{\mathrm{H}} U,
$$


where $U$ is a neighbourhood of zero in the horizontal tangent space at $x$ (i.e. in the orthogonal complement of $T_{x} \mathrm{G} x$ with respect to some G-invariant metric). Let $\phi: V \rightarrow W$ be as in Lemma C.2. Then the map

$$
\mathrm{G} \times U \times V \rightarrow \mathrm{G} \times U \times W:(g, u, v) \mapsto(g, u, g \phi(v))
$$

descends to a G-equivariant injective bundle homomorphism from $(\mathrm{G} \times U \times V) / \mathrm{H}$ to $\left(\mathrm{G} \times \times_{\mathrm{H}} U\right) \times W$ (where $\mathrm{G}$ acts diagonally). This construction gives rise to a G-invariant open cover $\left\{U_{\alpha}\right\}_{\alpha}$ of $X$ and a collection of G-equivariant injective bundle homomorphisms $\phi_{\alpha}:\left.E\right|_{U_{\alpha}} \rightarrow U_{\alpha} \times W_{\alpha}$. Let $\rho_{\alpha}: X \rightarrow[0,1]$ be a G-invariant partition of unity subordinate to the cover $\left\{U_{\alpha}\right\}_{\alpha}$ and denote $W:=\bigoplus_{\alpha} W_{\alpha}$. Then the homomorphism $E \rightarrow X \times W:(x, e) \mapsto\left(x,\left\{\rho_{\alpha}(x) \phi_{\alpha}(x) e\right\}_{\alpha}\right)$ is the required G-equivariant embedding. This proves the proposition.

\section{Appendix D. Convex polytopes}

In this section we recall some well-known facts about convex polytopes (see e.g. [9]). Let $\Delta$ be a compact convex polytope in the dual space $V^{*}$ of a finite dimensional vector space $V$. We denote elements of $V$ by $v, w$ and elements of $V^{*}$ by $\xi, \eta$. Define the support function $\phi: V \rightarrow \mathbb{R}$ of $\Delta$ by

$$
\phi(v):=\inf _{\xi \in \Delta}\langle\xi, v\rangle .
$$

The following properties of $\phi$ are obvious from the definition.

(P1) $\phi(t v)=t \phi(v)$ for $t \geq 0$.

(P2) $\phi$ is concave, i.e. $\phi(v+w) \geq \phi(v)+\phi(w)$.

(P3) $\Delta$ can be recovered from $\phi$ as the intersection of half spaces

$$
\Delta=\bigcap_{v \in V}\left\{\xi \in V^{*} \mid\langle\xi, v\rangle \geq \phi(v)\right\} .
$$

Let $F$ be a face of $\Delta$. Pick an interior point $p$ of $F$ and define the dual cone to $F$ by

$$
\check{F}:=\{v \in V \mid\langle\xi-p, v\rangle \geq 0 \text { for all } \xi \in \Delta\} .
$$

If $q$ is another interior point of $F$ and $\xi \in \Delta$ then $q+t(\xi-p) \in \Delta$ for $t>0$ sufficiently small. Hence $\langle\xi-p, v\rangle \geq 0$ iff $\langle q+t(\xi-p)-q, v\rangle \geq 0$. This shows that the definition of $\breve{F}$ does not depend on the point $p$. Moreover, the condition $\langle\xi-p, v\rangle \geq 0$ for all $\xi \in \Delta$ can be rewritten as $\phi(v) \geq\langle p, v\rangle$, or equivalently $\phi(v)=\langle p, v\rangle$ since $p \in \Delta$. So $\breve{F}$ can be written in the equivalent forms

$$
\begin{aligned}
\check{F} & =\{v \in V \mid\langle\xi-p, v\rangle \geq 0 \text { for all } \xi \in \Delta, p \in F\} \\
& =\{v \in V \mid\langle p, v\rangle=\phi(v) \text { for all } p \in F\} .
\end{aligned}
$$

The following properties are obvious from these descriptions of $\check{F}$. 
(F1) $\check{F}$ is a convex polyhedral cone.

(F2) The restriction of $\phi$ to $\breve{F}$ is the linear function $\phi(v)=\langle p, v\rangle$ for any $p \in F$.

(F3) $\check{F}$ is perpendicular to $F$ and $\operatorname{dim} \check{F}=\operatorname{codim} F$.

(F4) If $H_{1}, \ldots, H_{\ell} \subset V^{*}$ are the supporting hyperplanes for $\Delta$ meeting at $F$, then $\breve{F}$ is the cone generated by inward pointing normal vectors $v_{1}, \ldots, v_{\ell}$ to the hyperplanes.

(F5) If $G$ is a subface of $F$ then $\check{F}$ is a subcone of $\check{G}$.

(F6) The union of the cones $\breve{p}$ dual to vertices $p$ of $\Delta$ is the whole space $V$.

The collection $\Sigma$ of the cones $\breve{F}$ dual to nonempty faces of $\Delta$ is called the fan dual to $\Delta$ (see [3] for the general definition of a fan).

\section{Appendix E. The cohomology of symplectic quotients}

Let $T$ be a $k$-dimensional torus and $\rho=\left(\rho_{1}, \ldots, \rho_{n}\right): T \rightarrow \mathbb{T}^{n}:=\left(S^{1}\right)^{n}$ be a diagonal homomorphism with

$$
\rho_{\nu}(\exp (\xi))=e^{-2 \pi i\left\langle\mathrm{w}_{\nu}, \xi\right\rangle}
$$

for $\xi \in \mathfrak{t}:=\operatorname{Lie}(T)$. Here the $\mathrm{w}_{v}$ are elements of the dual lattice $\Lambda^{*} \subset \mathfrak{t}^{*}$ as in the introduction. We identify the Lie algebra of $\mathbb{T}^{n}$ with $\mathbb{R}^{n}$ via the map $\eta \mapsto i \eta / 2 \pi$ so that the integer lattice corresponds to $\mathbb{Z}^{n} \subset \mathbb{R}^{n}$. In this identification the linearization of $\rho$ is the map $\dot{\rho}: \mathfrak{t} \rightarrow \mathbb{R}^{n}$ given by

$$
\dot{\rho}(\xi)=\left(\left\langle\mathrm{w}_{1}, \xi\right\rangle, \ldots,\left\langle\mathrm{w}_{n}, \xi\right\rangle\right) .
$$

Consider the quotient torus

$$
\bar{T}:=\mathbb{T}^{n} / \rho(T) .
$$

Its Lie algebra is the quotient space

$$
\overline{\mathfrak{t}}:=\mathbb{R}^{n} / \dot{\rho}(\mathfrak{t})
$$

and the dual space of $\overline{\mathfrak{t}}$ can be identified with the subspace

$$
\overline{\mathfrak{t}}^{*}:=\left\{\eta \in\left(\mathbb{R}^{n}\right)^{*} \mid \sum_{\nu=1}^{n} \eta_{\nu} \mathrm{w}_{\nu}=0\right\} .
$$

The canonical action of $\mathbb{T}^{n}$ on $\mathbb{C}^{n}$ induces an action of $T$ with moment map $\mu: \mathbb{C}^{n} \rightarrow \mathfrak{t}^{*}$ given by (1). We assume throughout that $\mu$ is proper and that the action is effective (i.e. the weight vectors $\mathrm{w}_{v}$ span $\mathfrak{t}^{*}$ ). Let $\tau \in \mathfrak{t}^{*}$ be a regular value of $\mu$. Then the torus $\bar{T}$ acts on the symplectic quotient

$$
\bar{M}:=\mathbb{C}^{n} / / T(\tau)=\mu^{-1}(\tau) / T .
$$


A moment map $\bar{\mu}: \bar{M} \rightarrow \overline{\mathfrak{t}}^{*}$ for this action is given by the formula

$$
\bar{\mu}(x):=\left(\begin{array}{c}
\pi\left|x_{1}\right|^{2}+\zeta_{1} \\
\vdots \\
\pi\left|x_{n}\right|^{2}+\zeta_{n}
\end{array}\right),
$$

where $\zeta=\left(\zeta_{1}, \ldots, \zeta_{n}\right) \in\left(\mathbb{R}^{n}\right)^{*}$ is chosen such that

$$
\sum_{\nu=1}^{n} \zeta_{\nu} \mathrm{w}_{\nu}=-\tau
$$

The image of $\bar{\mu}$ is the convex polyhedron

$$
\Delta:=\bar{\mu}(\bar{M})=\left\{\eta \in\left(\mathbb{R}^{n}\right)^{*} \mid \sum_{\nu=1}^{n} \eta_{\nu} \mathrm{w}_{\nu}=0, \eta_{\nu} \geq \zeta_{\nu}\right\} .
$$

Each subset $I \subset\{1, \ldots, n\}$ determines a (possibly empty) face

$$
\Delta_{I}:=\left\{\eta \in \Delta \mid \eta_{\nu}=\zeta_{\nu} \text { for } v \in I\right\} .
$$

Recall that $C(I)$ denotes the cone spanned by the vectors $\mathrm{w}_{v}, v \in I$. The next lemma shows that if $\tau$ is a regular value of $\mu$, then the intersection of any $j$ codimension- 1 faces of $\Delta$ is either empty or has codimension $j$.

Lemma E.1. Assume that $\tau$ is a regular value of $\mu$ and let $I \subset\{1, \ldots, n\}$.

(i) The set $\Delta_{I}$ is either empty or has codimension $|I|$.

(ii) $\Delta_{I}=\emptyset \Longleftrightarrow \tau \notin C(\{1, \ldots, n\} \backslash I)$.

Proof. We prove (i). Assume $\Delta_{I} \neq \emptyset$ and let $J:=\{1, \ldots, n\} \backslash I$. Then, by the definition of $\bar{\mu}$, there is a $y \in \mathbb{C}^{J}$ such that

$$
\mu(y)=\pi \sum_{\nu \in J}\left|y_{v}\right|^{2} \mathrm{w}_{v}=\tau .
$$

Since $\tau$ is a regular value of $\mu$, there exist indices $v_{1}, \ldots, v_{k} \in J$ such that the vectors $\mathrm{w}_{v_{1}}, \ldots, \mathrm{w}_{v_{k}}$ are linearly independent and $y_{v_{j}} \neq 0$ for every $j$. We claim that there is a vector $x \in \mathbb{C}^{J}$ such that

$$
\mu(x)=\tau, \quad x_{v} \neq 0 \text { for all } v \in J .
$$

To see this choose $x_{v}$ for $v \in J \backslash\left\{v_{1}, \ldots, v_{k}\right\}$ such that $\left|x_{v}\right|^{2}=\left|y_{v}\right|^{2}+\varepsilon$ and choose $x_{v_{j}}$ such that

$$
\sum_{j=1}^{k}\left(\left|x_{v_{j}}\right|^{2}-\left|y_{v_{j}}\right|^{2}\right) \mathrm{w}_{v_{j}}+\varepsilon \sum_{\nu \in J \backslash\left\{v_{1}, \ldots, v_{k}\right\}} \mathrm{w}_{\nu}=0 .
$$


Then $\mu(x)=\mu(y)=\tau$ and, for $\varepsilon>0$ sufficiently small, we get $x_{v} \neq 0$ for all $v \in J$.

The differential $d \bar{\mu}(x): T_{x}\left(\bar{M} \cap \mathbb{C}^{J}\right) \rightarrow \overline{\mathfrak{t}}^{*}$ is given by

$$
d \bar{\mu}(x) v=\left(2 \pi\left\langle x_{v}, v_{\nu}\right\rangle\right)_{v \in J},
$$

where $v \in \mathbb{C}^{J}$ satisfies

$$
d \mu(x) v=2 \pi \sum_{\nu \in J}\left\langle x_{v}, v_{v}\right\rangle \mathrm{w}_{v}=0 .
$$

Since $x_{v} \neq 0$ for $v \in J$, this shows that the image of $T_{x}\left(\bar{M} \cap \mathbb{C}^{J}\right)$ under $d \bar{\mu}(x)$ equals $\left\{\eta \in\left(\mathbb{R}^{J}\right)^{*} \mid \sum_{v \in J} \eta_{v} \mathrm{w}_{v}=0\right\}$. This space, and therefore $\Delta_{I}$, has dimension $|J|-k=n-k-|I|$.

We prove (ii). If $\Delta_{I} \neq \emptyset$ there exists an $\eta \in\left(\mathbb{R}^{n}\right)^{*}$ such that

$$
\sum_{\nu=1}^{n} \eta_{\nu} \mathrm{w}_{v}=0, \quad \eta_{v} \geq \zeta_{v}
$$

for all $v$, and

$$
v \in I \quad \Longrightarrow \quad \eta_{v}=\zeta_{v}
$$

Hence

$$
\tau=\sum_{\nu=1}^{n}-\zeta_{\nu} w_{\nu}=\sum_{\nu \notin I}\left(\eta_{\nu}-\zeta_{\nu}\right) \mathrm{w}_{\nu} \in C(\{1, \ldots, n\} \backslash I) .
$$

The converse follows by reversing the argument.

Standing assumption. In the remainder of this appendix we assume that $T$ acts freely on $\mu^{-1}(\tau)$.

Denote by $\overline{\mathrm{w}}_{v} \in H^{2}(\bar{M} ; \mathbb{R})$ the image of $\mathrm{w}_{v}$ under the homomorphism $\Lambda^{*} \cong$ $H^{2}(\mathrm{BT} ; \mathbb{Z}) \rightarrow H^{*}(\bar{M} ; \mathbb{R})$.

Lemma E.2. For every $J \subset\{1, \ldots, n\}$ the following holds.

(i) If $\tau \notin C(J)$ then $\prod_{v \notin J} \overline{\mathrm{w}}_{v}=0$.

(ii) If $\tau \in C(J)$ and $|J|=k$ then $\prod_{v \notin J} \overline{\mathrm{w}}_{v}=\mathrm{PD}(\mathrm{pt})$.

Proof. $\overline{\mathrm{W}}_{v}$ is the first Chern class of the line bundle $\bar{L}_{v}:=\mu^{-1}(\tau) \times_{\rho_{v}} \mathbb{C}$. Hence the zero set of the holomorphic section $\bar{M} \rightarrow \bar{L}_{v}:[x] \mapsto\left[x, x_{v}\right]$ is Poincaré dual to $\overline{\mathrm{W}}_{v}$. Denote this zero set by $\bar{W}_{v}:=\left\{[x] \in \bar{M} \mid x_{v}=0\right\}$. This is a (possibly empty) complex submanifold of $\bar{M}$ of complex codimension one. Moreover,

$$
\tau \notin C(J) \Longrightarrow \bigcap_{\nu \notin J} \bar{W}_{v}=\emptyset
$$

This proves (i). If $\tau \in C(J)$ and $|J|=k$, then the submanifolds $\overline{\mathrm{W}}_{v}$ for $v \notin J$ intersect transversally in a single point. This proves (ii). 
Lemma E.3. (i) The Chern classes of $T \bar{M}$ are given by

$$
c_{j}(T \bar{M})=\sum_{\nu_{1}<\cdots<v_{j}} \overline{\mathrm{w}}_{\nu_{1}} \cdots \overline{\mathrm{w}}_{\nu_{j}}, \quad j=1, \ldots, n-k .
$$

(ii) The cohomology class of the symplectic form $\bar{\omega} \in \Omega^{2}(\bar{M})$ is

$$
[\bar{\omega}]=\bar{\tau} .
$$

Proof. We prove (i). Consider the Whitney sum $\mu^{-1}(\tau) \times \mathbb{C}^{n}=E \oplus F$, where the complex vector bundles $E \rightarrow \mu^{-1}(\tau)$ and $F \rightarrow \mu^{-1}(\tau)$ are defined by

$$
\begin{aligned}
& E_{x}:=\left\{v \in \mathbb{C}^{n} \mid d \mu(x) v=d \mu(x) i v=0\right\}, \\
& F_{x}:=\left\{v \in \mathbb{C}^{n} \mid \exists \xi, \eta \in \mathfrak{t} \forall v: v_{v}=\left(\left\langle\mathrm{w}_{\nu}, \xi\right\rangle+i\left\langle\mathrm{w}_{v}, \eta\right\rangle\right) x_{v}\right\} .
\end{aligned}
$$

Then the bundle $F$ admits a $T$-equivariant complex trivialization and the quotient bundle $E / T \rightarrow \mu^{-1}(\tau) / T$ is isomorphic to the tangent bundle of $\bar{M}$. Hence

$$
c_{j}(T \bar{M})=c_{j}\left(\mu^{-1}(\tau) \times_{T} \mathbb{C}^{n}\right)=\sum_{\nu_{1}<\cdots<v_{j}} \overline{\mathrm{w}}_{\nu_{1}} \cdots \overline{\mathrm{w}}_{v_{j}} .
$$

We prove (ii). Denote

$$
\lambda_{0}:=\frac{1}{2 i} \sum_{\nu=1}^{n}\left(\bar{x}_{\nu} d x_{v}-x_{\nu} d \bar{x}_{v}\right) \in \Omega^{1}\left(\mathbb{C}^{n}\right), \quad \omega_{0}:=d \lambda_{0},
$$

and let $\mu_{0}: \mathbb{C}^{n} \rightarrow \mathbb{R}^{n}$ be the moment map given by

$$
\mu_{0}(x):=\pi\left(\left|x_{1}\right|^{2}, \ldots,\left|x_{n}\right|^{2}\right) .
$$

Since $d_{\mathbb{T}^{n}} \lambda_{0}=\omega_{0}-\mu_{0}$, the equivariant cohomology class $\left[\omega_{0}-\mu_{0}\right] \in H_{\mathbb{T}^{n}}^{2}\left(\mathbb{C}^{n}\right)$ is trivial. Pulling back under the homomorphism $H_{\mathbb{T}^{n}}^{2}\left(\mathbb{C}^{n}\right) \rightarrow H_{T}^{2}\left(\mathbb{C}^{n}\right)$ induced by $\rho$ yields $0=\left[\omega_{0}-\mu\right] \in H_{T}^{2}\left(\mathbb{C}^{n}\right)$. Restriction to $\mu^{-1}(\tau)$ yields $0=\left[\iota^{*} \omega_{0}-\iota^{*} \tau\right]$ $\in H_{T}^{2}\left(\mu^{-1}(\tau)\right)$, where $\iota: \mu^{-1}(\tau) \rightarrow \mathbb{C}^{n}$ is the inclusion. Now the result follows by passing to the quotient.

Theorem E.4 ([12]). The ring homomorphism

$$
\mathbb{R}\left[u_{1}, \ldots, u_{n}\right] \rightarrow H^{*}(\bar{M} ; \mathbb{R}): p\left(u_{1}, \ldots, u_{n}\right) \mapsto p\left(\overline{\mathrm{w}}_{1}, \ldots, \overline{\mathrm{w}}_{n}\right)
$$

induces an isomorphism

$$
H^{*}(\bar{M} ; \mathbb{R}) \cong \mathbb{R}\left[u_{1}, \ldots, u_{n}\right] / \mathcal{I},
$$

where the ideal $\mathcal{I} \subset \mathbb{R}\left[u_{1}, \ldots, u_{n}\right]$ is generated by the relations

$$
\begin{aligned}
& \sum_{\nu=1}^{n} \eta_{\nu} \mathrm{w}_{\nu}=0 \Longrightarrow \sum_{\nu=1}^{n} \eta_{\nu} u_{v}=0, \\
& I \subset\{1, \ldots, n\}, \quad \Delta_{I}=\emptyset \Longrightarrow \prod_{v \in I} u_{v}=0 .
\end{aligned}
$$


Remark. Theorem E.4 continues to hold with coefficients in $\mathbb{Z}$. That the homomorphism (59) is surjective follows from Kirwan's theorem, and that the ideal $\mathcal{I}$ is contained in the kernel of (59) is an easy consequence of Lemma E.2. The nontrivial part of the proof is to show that the kernel is contained in $\mathcal{I}$.

Theorem E.5 ([4]). Assume $\mu^{-1}(\tau) \neq \emptyset$ and $T$ acts freely on $\mu^{-1}(\tau)$. Then the ideal $\mathcal{I}$ is generated by the linear relations (60), the linear monomials $u_{v}$ for $\Delta_{\{v\}}=\emptyset$, and the monomials $u^{d^{+}}$for $d \in \mathcal{D}_{\text {eff }}(\tau) \backslash\{0\}$.

For the sake of completeness, we present a somewhat more elaborated version of the proof given in [4]. We need some preparation. Denote by $\bar{\Lambda} \subset \overline{\mathfrak{t}}$ and $\bar{\Lambda}^{*} \subset \overline{\mathfrak{t}}^{*}$ the integer lattices. Thus $\bar{\Lambda}$ is the image of $\mathbb{Z}^{n}$ under the projection $\mathbb{R}^{n} \rightarrow \overline{\mathrm{t}}$ and $\bar{\Lambda}^{*}=\overline{\mathfrak{t}}^{*} \cap\left(\mathbb{Z}^{n}\right)^{*}$. For $v=1, \ldots, n$ let $\bar{e}_{v} \in \bar{\Lambda}$ be the image of the basis vector $e_{v}=(0, \ldots, 0,1,0, \ldots, 0) \in \mathbb{Z}^{n}$ under the projection $\mathbb{R}^{n} \rightarrow \overline{\mathfrak{t}}$.

Lemma E.6. Suppose that $T$ acts freely on $\mu^{-1}(\tau)$.

(i) Let $J \subset\{1, \ldots, n\}$ satisfy $|J|=n-k$ and $\Delta_{J} \neq \emptyset$. Then the vectors $\left\{\bar{e}_{j} \mid j \in J\right\}$ form an integer basis of $\bar{\Lambda}$.

(ii) Let $d_{v} \in \mathbb{Z}$ satisfy $\sum_{v=1}^{n} d_{v} \bar{e}_{v}=0$. Then there exists a vector $\lambda \in \Lambda$ such that $d_{v}=\left\langle\mathrm{w}_{\nu}, \lambda\right\rangle$ for every $v$.

Proof. We prove (i). Assume $|J|=n-k$ and $\Delta_{J} \neq \emptyset$. Since $T$ acts freely on $\mu^{-1}(\tau)$, and $\tau \in C(\{1, \ldots, n\} \backslash J)$, the vectors $\left\{\mathrm{w}_{\nu} \mid \nu \notin J\right\}$ form an integer basis of $\Lambda^{*}$. Hence, for every $v \in \mathbb{Z}^{n}$, there exists a unique vector $\lambda \in \Lambda$ such that $v_{v}=\left\langle\mathrm{w}_{v}, \lambda\right\rangle$ for $v \notin J$. This implies that the image $\bar{v} \in \overline{\mathfrak{t}}$ of $v$ under the projection $\mathbb{R}^{n} \rightarrow \overline{\mathfrak{t}}$ satisfies

$$
\bar{v}=\sum_{j \in J}\left(v_{j}-\left\langle\mathrm{w}_{j}, \lambda\right\rangle\right) \bar{e}_{j} .
$$

Hence the vectors $\left\{\bar{e}_{j} \mid j \in J\right\}$ span the integer lattice $\bar{\Lambda}$ as claimed.

We prove (ii). By definition of the projection $\mathbb{R}^{n} \rightarrow \overline{\mathfrak{t}}$, there exists a vector $\xi \in \mathfrak{t}$ such that $d_{v}=\left\langle\mathrm{w}_{v}, \xi\right\rangle$ for every $v$. Now let $J \subset\{1, \ldots, n\}$ be any index set such that $|J|=n-k$ and $\Delta_{J} \neq \emptyset$. Then the argument in the proof of (i) shows that there exists a lattice vector $\lambda \in \Lambda$ such that $d_{v}=\left\langle\mathrm{w}_{\nu}, \lambda\right\rangle$ for $v \notin J$. Hence $\left\langle\mathrm{w}_{v}, \xi-\lambda\right\rangle=0$ for $v \notin J$. Since the vectors $\left\{\mathrm{w}_{v} \mid v \notin J\right\}$ form a basis of $\mathrm{t}^{*}$ we deduce that $\xi=\lambda$ and hence $d_{v}=\left\langle\mathrm{w}_{\nu}, \lambda\right\rangle$ for every $\nu$.

Proof of Theorem E.5. Let $\mathcal{I}_{0} \subset \mathbb{R}\left[u_{1}, \ldots, u_{n}\right]$ be the ideal generated by the linear polynomials $\sum_{v=1}^{n} \eta_{v} u_{v}$, where $\sum_{v=1}^{n} \eta_{v} \mathrm{w}_{v}=0$, the monomials $u_{v}$, where $\Delta_{\{\nu\}}=\emptyset$, and the monomials $u^{d^{+}}$for $d \in \mathcal{D}_{\text {eff }}(\tau) \backslash\{0\}$.

We prove that $\mathcal{I}_{0} \subset \mathcal{I}$. We must show that $u^{d^{+}} \in \mathcal{I}$ for every $d \in \mathcal{D}_{\text {eff }}(\tau) \backslash\{0\}$. We prove a stronger statement: If $\lambda \in \Lambda$ satisfies $\langle\tau, \lambda\rangle>0$ and $d_{v}:=\left\langle\mathrm{w}_{v}, \lambda\right\rangle$ then $u^{d^{+}} \in \mathcal{I}$. To see this, consider the set $I:=\left\{v \mid d_{v}>0\right\}$. We claim that 
$\Delta_{I}=\emptyset$. Otherwise, by Lemma E.1, there would exist numbers $\eta_{v} \geq 0$ such that $\tau=\sum_{v \notin I} \eta_{v} \mathrm{w}_{v}$. But then

$$
0<\langle\tau, \lambda\rangle=\sum_{\nu \notin I} \eta_{\nu} d_{\nu} \leq 0,
$$

a contradiction. Since $\Delta_{I}=\emptyset$, the monomial $\prod_{v \in I} u_{v}$ belongs to the ideal $\mathcal{I}$. But $u^{d^{+}}$is a multiple of $\prod_{v \in I} u_{v}$ and hence also belongs to $\mathcal{I}$.

We prove that $\mathcal{I} \subset \mathcal{I}_{0}$. Consider the moment polytope $\Delta \subset \overline{\mathfrak{t}}^{*}$ defined by (58). The faces of $\Delta$ are subsets of the form $\Delta_{I}$ for $I \subset\{1, \ldots, n\}$ such that $\tau \in$ $C(\{1, \ldots, n\} \backslash I)$. The vectors $\left\{\bar{e}_{i} \mid i \in I\right\}$ are the inward pointing normal vectors to the supporting hyperplanes of $\Delta$ meeting at the face $\Delta_{I}$. Hence, by property $(\mathrm{F} 4)$ of the dual cones (see Appendix D), the dual cone of $\Delta_{I}$ is given by

$$
\check{\Delta}_{I}=\left\{\sum_{i \in I} c_{i} \bar{e}_{i} \mid c_{i} \geq 0\right\} .
$$

By Lemma E.1, the codimension of the face $\Delta_{I}$ equals $|I|$. In particular, the vertices of $\Delta$ are subsets $\Delta_{J}$ where $|J|=n-k$ and $\Delta_{J} \neq \emptyset$.

Now let $I \subset\{1, \ldots, n\}$ such that $\Delta_{I}=\emptyset$. We must prove that the monomial $\prod_{v \in I} u_{v}$ belongs to $\mathcal{I}_{0}$. Shrinking the set $I$, if necessary, we may assume without loss of generality that $\Delta_{I^{\prime}} \neq \emptyset$ for every proper subset $I^{\prime} \subsetneq I$. Since $\mu(\tau) \neq \emptyset$ we have $I \neq \emptyset$. If $|I|=1$ then the polynomial $\prod_{v \in I} u_{v}$ belongs to $\mathcal{I}_{0}$ by assumption. Hence assume $|I| \geq 2$. Then

$$
v \in I \quad \Longrightarrow \quad \Delta_{\{v\}} \neq \emptyset
$$

We shall prove that there exists a vector $d \in \mathcal{D}_{\text {eff }}(\tau) \backslash\{0\}$ such that $d_{v}=1$ for $v \in I$ and $d_{v} \leq 0$ for $v \notin I$. To see this, consider the vector $\sum_{i \in I} \bar{e}_{i} \in \bar{\Lambda}$. Since the union of the cones dual to vertices is the whole space $\overline{\mathfrak{t}}$, it follows that there exists an index set $J \subset\{1, \ldots, n\}$ such that $|J|=n-k, \Delta_{J} \neq \emptyset$, and $\sum_{i \in I} \bar{e}_{i} \in \check{\Delta}_{J}$. Hence there exists nonnegative real numbers $c_{j}$ such that

$$
\sum_{i \in I} \bar{e}_{i}=\sum_{j \in J} c_{j} \bar{e}_{j}
$$

By Lemma E.6, the set $\left\{\bar{e}_{j} \mid j \in J\right\}$ is an integer basis of $\bar{\Lambda}$. Hence the $c_{j}$ are actually integers and, after shrinking $J$, we may assume that $c_{j}>0$ for all $j \in J$. Define $d \in \mathbb{Z}^{n}$ by

$$
d_{v}:=\left\{\begin{aligned}
1, & \text { if } v \in I \backslash J, \\
-c_{v}, & \text { if } v \in J \backslash I, \\
1-c_{v}, & \text { if } v \in I \cap J, \\
0, & \text { if } v \notin I \cup J
\end{aligned}\right.
$$


Then $\sum_{v=1}^{n} d_{\nu} \bar{e}_{\nu}=0$ and hence, by Lemma E.6, there exists a lattice vector $\lambda \in \Lambda$ such that $d_{v}=\left\langle\mathrm{w}_{v}, \lambda\right\rangle$ for $v=1, \ldots, n$. By (62), we have $\Delta_{\{v\}} \neq \emptyset$ for every $v \in I \cup J$. Hence $d_{v}=0$ whenever $\Delta_{\{v\}}=\emptyset$, and this implies $\lambda \in \Lambda(\tau)$.

We prove that $d \in \mathcal{D}_{\text {eff }}(\tau) \backslash\{0\}$. Let $\phi: \overline{\mathfrak{t}} \rightarrow \mathbb{R}$ be the support function of $\Delta$ as in Appendix D. By property (P2), we have

$$
\sum_{i \in I} \phi\left(\bar{e}_{i}\right) \leq \phi\left(\sum_{i \in I} \bar{e}_{i}\right)=\phi\left(\sum_{j \in J} c_{j} \bar{e}_{j}\right)=\sum_{j \in J} c_{j} \phi\left(\bar{e}_{j}\right) .
$$

Here the last equation follows from property (F2) and the fact that the set $\left\{\bar{e}_{j} \mid j\right.$ $\in J\}$ spans the cone $\check{\Delta}_{J}$. Now, by (58) and the definition of $\phi$, we have $\phi\left(\bar{e}_{\nu}\right) \geq \zeta_{\nu}$, with equality if and only if the face $\Delta_{\{v\}}$ is nonempty. Moreover, $d_{v}=0$ whenever $\Delta_{\{v\}}=\emptyset$. This implies

$$
0 \geq \sum_{\nu=1}^{n} d_{\nu} \phi\left(\bar{e}_{\nu}\right)=\sum_{\nu=1}^{n} d_{\nu} \zeta_{\nu}=\sum_{\nu=1}^{n}\left\langle\mathrm{w}_{\nu}, \lambda\right\rangle \zeta_{\nu}=-\langle\tau, \lambda\rangle .
$$

If we replace $\tau$ by another vector $\tau^{\prime}$ in the same chamber, the fan $\Sigma$ remains the same, so the above argument yields the same vector $\lambda \in \Lambda$. This shows that $\left\langle\tau^{\prime}, \lambda\right\rangle \geq 0$ for every $\tau^{\prime}$ in the chamber of $\tau$. So $\lambda \in \Lambda_{\text {eff }}(\tau)$ and $d \in \mathcal{D}_{\text {eff }}(\tau)$. Since $\Delta_{I}=\emptyset$ and $\Delta_{J} \neq \emptyset$, we have $I \neq J$ and hence $d \neq 0$.

We prove that $I \cap J=\emptyset$. Otherwise let $v_{0} \in I \cap J$ and $I^{\prime}:=I \backslash\left\{v_{0}\right\}$. Then $d_{v} \leq 0$ for $v \notin I^{\prime}$. Hence the argument in the proof of $\mathcal{I}_{0} \subset \mathcal{I}$ shows that $\Delta_{I^{\prime}}=\emptyset$. But this contradicts the minimality assumption on $I$. Hence $I \cap J=\emptyset$ as claimed. It follows that the vector $d$ satisfies $d_{v}=1$ for $v \in I$ and $d_{v} \leq 0$ for $v \notin I$. Since $d \in \mathcal{D}_{\text {eff }}(\tau) \backslash\{0\}$ we deduce that

$$
\prod_{v \in I} u_{v}=u^{d^{+}} \in \mathcal{I}_{0} .
$$

This implies $\mathcal{I} \subset \mathcal{I}_{0}$ and hence $\mathcal{I}=\mathcal{I}_{0}$.

\section{References}

1. Atiyah, M.F., Singer, I.: The index of elliptic operators: III. Annals of Mathematics 87, 546-604 (1968)

2. Atiyah, M.F., Singer, I.: The index of elliptic operators: IV. Annals of Mathematics 93, 119-138 (1971)

3. Audin, M.: The Topology of Torus Actions on Symplectic Manifolds, Progress in Mathematics 93. Birkhäuser, Basel, 1991

4. Batyrev, V.: Quantum cohomology rings of toric manifolds, Journées de Géométrie Algébrique d'Orsay (Orsay, 1992), Astérisque 218, 9-34 (1993)

5. Berline, N., Vergne, M.: Zeros d'un champ de vecteurs et classes characteristiques equivariantes, Duke Math. Journal 50, 539-549 (1983) 
6. Berline, N., Getzler, E., Vergne, M.: Heat Kernels and Dirac Operators. Springer Verlag, Grundlagen der Mathematischen Wissenschaften, 298

7. Cieliebak, K., Gaio, A.R., Mundet i Riera. I., Salamon, D.A.: The symplectic vortex equations and invariants of Hamiltonian group actions. The Journal of Symplectic Geometry $\mathbf{3}$, 543-645 (2002)

8. Cieliebak, K., Mundet i Riera, I., Salamon, D.A.: Equivariant moduli problems and the Euler class. Topology 42, 641-700 (2003)

9. Oda, T.: Convex Bodies and Algebraic Geometry, Ergebnisse der Mathematik und ihrer Grenzgebiete, 3. Folge Band 14, Springer, Berlin 1985

10. Gaio, A.R., Salamon, D.A.: Gromov-Witten invariants of symplectic quotients and adiabatic limits, Preprint ETH-Zürich, June 2001. To appear in The Journal of Symplectic Geometry

11. Givental, A.B.: A mirror theorem for toric complete intersections, in Topological Field Theory, Primitive Forms, and Related Topics. Kyoto, 1996, pp. 141-175, Progr. Math. 160, Birkhäser, Boston 1998

12. Guillemin, V., Sternberg, S.: Supersymmetry and Equivariant de Rham theory. Springer, Berlin 1999

13. McDuff, D., Salamon, D.: $J$-holomorphic Curves and Symplectic Topology. AMS Colloquium Publications, Vol. 52, 2004

14. Okonek, C., Teleman, A.: Gauge theoretic equivariant Gromov-Witten invariants and the full Seiberg-Witten invariants of ruled surfaces. Comm. Math. Phys 227, 551-585 (2002)

15. Qin, Z., Ruan, Y.: Quantum cohomology of projective bundles over $\mathbb{P}^{n}$, Trans. Amer. Math. Soc. 350, 3615-3638 (1998)

16. Spielberg, H.: The Gromov-Witten invariants of symplectic toric manifolds, Preprint, arXiv:math.AG/0006156, November 1999

17. Spielberg, H.: Counting generic genus-0 curves on Hirzebruch surfaces. Proc. Amer. Math. Soc. 130, 1257-1264 (2002) 